\title{
Polar Dust, Nuclear Obscuration, and IR SED Diversity in Type-1 AGNs*
}

\author{
Jianwei Lyu (吕建伟) (10) and George H. Rieke (i) \\ Steward Observatory, University of Arizona, 933 North Cherry Avenue, Tucson, AZ 85721, USA; jianwei@email.arizona.edu \\ Received 2018 May 15; revised 2018 August 29; accepted 2018 September 8; published 2018 October 16
}

\begin{abstract}
Despite the hypothesized similar face-on viewing angles, the infrared emission of type- 1 active galactic nuclei (AGNs) has diverse spectral energy distribution (SED) shapes that deviate substantially from the wellcharacterized quasar templates. Motivated by the commonly seen UV-optical obscuration and the discovery of parsec-scale mid-IR polar dust emission in some nearby AGNs, we develop semi-empirical SED libraries for reddened type-1 AGNs built on quasar intrinsic templates, assuming low-level extinction caused by an extended distribution of large dust grains. We demonstrate that this model can reproduce the nuclear UV to IR SED and the strong mid-IR polar dust emission of NGC 3783, the type-1 AGN with the most relevant and robust observational constraints. In addition, we compile 64 low- $z$ Seyfert-1 nuclei with negligible mid-IR star formation contamination and satisfactorily fit the individual IR SEDs as well as the composite UV to mid-IR composite SEDs. Given the success of these fits, we characterize the possible infrared SED of AGN polar dust emission and utilize a simple but effective strategy to infer its prevalence among type-1 AGNs. The SEDs of high- $z$ peculiar AGNs, including the extremely red quasars, mid-IR warm-excess AGNs, and hot dust-obscured galaxies, can be also reproduced by our model. These results indicate that the IR SEDs of most AGNs, regardless of redshift or luminosity, arise from similar circumnuclear torus properties but differ mainly due to the optical depths of extended obscuring dust components.
\end{abstract}

Key words: dust, extinction - galaxies: active - galaxies: Seyfert - infrared: galaxies - quasars: general

\section{Introduction}

Accreting supermassive black holes (BHs) are surrounded by copious dust and generally emit strongly in the infrared (IR). Compared with star-forming or quiescent galaxies whose emission is dominated by stars and $\mathrm{H}$ II regions, active galactic nuclei (AGNs) have distinctive IR spectral energy distributions (SEDs), offering a unique window to look for obscured AGNs (e.g., Lacy et al. 2004, 2007; Stern et al. 2005, 2012; Donley et al. 2012), a critical means to explore the properties of dusty structures around their central engines (e.g., Fritz et al. 2006; Nenkova et al. 2008a, 2008b; Stalevski et al. 2012, 2016), and a powerful tool to constrain their host galaxy properties by SED decompositions (e.g., Bongiorno et al. 2007, 2012; Xu et al. 2015; Lyu et al. 2016; Collinson et al. 2017). More importantly, the dusty structures around the central engine, commonly termed as "torus," lay the foundation of AGN unification and have physical scales that bridge the $\mathrm{BH}$ accretion disk and the host galaxy (e.g., Antonucci 1993; Urry \& Padovani 1995). IR SEDs have become a crucial probe for the AGN phenomenon and the BH-galaxy coevolution (e.g., Caputi 2014; Heckman \& Best 2014; Netzer 2015). Nevertheless, most studies are focused on individual objects or some particular narrowly defined AGN population. In this work, we propose and test a model to reconcile the various IR SEDs of type-1 AGNs over a broad range of luminosity and redshift.

The intrinsic SEDs of the most luminous AGNs, or quasars (bolometric luminosity $L_{\mathrm{AGN}, \text { bol }} \gtrsim 10^{11} L_{\odot}$ ), are observationally well characterized (e.g., Elvis et al. 1994; Richards et al. 2006; Shang et al. 2011; Krawczyk et al. 2013; Scott \& Stewart 2014; Lyu et al. 2017; Lyu \& Rieke 2017; Lani et al. 2017). The average SEDs of unobscured quasars, whether the

\footnotetext{
* The AGN template library developed in this work can be obtained on github (https://github.com/karlan/AGN_templates).
}

parent sample is (mainly) optically selected (e.g., Elvis et al. 1994; Krawczyk et al. 2013), or combined with mid-IR selection (e.g., Richards et al. 2006), or X-ray selected (e.g., Polletta et al. 2007; Elvis et al. 2012), have been found to be strikingly similar. The classical Elvis et al. (1994)-like template has proven to be a realistic representation of the IR emission by hot dust in most quasars (Lyu et al. 2017). The exceptions, including hot-dust-free or hot-dust-poor objects (e.g., Hao et al. 2010, 2011; Jiang et al. 2010), are part of the quasar intrinsic IR SED diversity seen at all redshifts. For unobscured quasars at $z \sim 0-6$, the AGN IR SEDs can be generally grouped into three basic types; besides the normal AGNs described by the Elvis-like template, there are also the warm-dust-deficient (WDD) AGNs and the hot-dust-deficient (HDD) AGNs (Lyu et al. 2017). Despite the variations in the near-IR or mid-IR, the shapes of quasar far-IR intrinsic SEDs have an identical pattern in a statistical sense (Lyu \& Rieke 2017), which drops quickly at $\lambda \gtrsim 20 \mu \mathrm{m}$ (Xu et al. 2015; Lyu \& Rieke 2017; see also Lani et al. 2017).

By comparison, the behavior of the intrinsic emission from relatively faint AGNs $\left(L_{\mathrm{AGN}, \text { bol }} \sim 10^{8}-10^{11} L_{\odot}\right)$ is elusive because of the significant galaxy contamination as well as the possible line-of-sight (LOS) extinction. A frequent hypothesis is that the intrinsic SEDs of these Seyfert nuclei are identical to those of quasars, thus one single template for AGNs with a very broad luminosity range is assumed (e.g., Hopkins et al. 2007; Assef et al. 2010; Donley et al. 2012). However, notable differences seem to exist, particularly in the IR. The AGN emission of many Seyfert-1 galaxies is peaked at $\lambda \gtrsim 10 \mu \mathrm{m}$ and the near- to mid-IR broad-band SEDs are commonly described by a single power law (e.g., Spinoglio et al. 1995; Alonso-Herrero et al. 2003; Prieto et al. 2010). On the other hand, normal quasars exhibit a prominent SED bump peaked in the UV and emit relatively less strongly in the IR with an obvious SED jump due to emission by hot dust starting 
at $1.3 \mu \mathrm{m}$ and an almost flat $\left(\nu F_{\nu} \propto \nu^{0}\right)$ mid-IR SED at 3-20 $\mu \mathrm{m}$ (e.g., Sanders et al. 1989; Elvis et al. 1994). As suggested by, e.g., Prieto et al. (2010), such different behavior might be caused by obscuration. However, frequently only the reddening of the UV-optical SED is considered, while the accompanying effect on the IR emission is ignored.

In fact, an equatorial optically thick torus is only a first-order approximation of the circumnuclear dust environment around an AGN. Evidence for polar dust at $\sim 10^{2}$ pc scale has been suggested since the early 1990s (e.g., Braatz et al. 1993; Cameron et al. 1993; Bock et al. 2000) and the AGN obscuration is known to happen at a range of different scales (e.g., see review by Bianchi et al. 2012). In theory, the AGN torus could form during the gas accretion of the central $\mathrm{BH}$ and have material exchanges with the ambient environment (e.g., Hopkins et al. 2012). Although it cannot survive very close to the central engine, dust is expected to be found in many other AGN components, e.g., narrow-line regions (NLRs) (e.g., Groves et al. 2006; Mor et al. 2009) and/or AGN-driven outflows (e.g., Fabian 1999; Murray et al. 2005). As argued by many authors, the AGN infrared SED might be easily reshaped by these extended dusty structures (e.g., Sturm et al. 2005; Groves et al. 2006; Hönig et al. 2012, 2013; Hönig \& Kishimoto 2017).

Mid-IR interferometric observations have become available for some nearby Seyfert nuclei, allowing a direct investigation of the geometry of their nuclear IR structures at parsec scales. Interestingly, these studies show that the mid-IR warm dust emission in some systems is largely distributed along the AGN polar direction, instead of from an equatorial torus (e.g., Raban et al. 2009; Hönig et al. 2012, 2013; Tristram et al. 2014; López-Gonzaga et al. 2016; Leftley et al. 2018). These observations have motivated the developments of increasingly sophisticated dust models to explain the few best-studied cases (Hönig \& Kishimoto 2017; Stalevski et al. 2017). In the case of NGC 3783, it is proposed that both the torus and the polar dust are composed of optically thick clouds and that the polar dust is composed of large carbon grains (Hönig \& Kishimoto 2017). In comparison, the Circinus Galaxy is modeled with a parsecscale optically thick dusty disk and an IR optically thin cone following the structure of the NLR out to a distance of $\sim 40 \mathrm{pc}$ (Stalevski et al. 2017). The success of these different approaches indicates that these complex models could be highly degenerate for most AGNs due to the lack of detailed observational constraints. We will address whether it is possible to develop a much simpler model that can be applied uniformly and is still of sufficient fidelity to provide useful insights.

With the success of our intrinsic templates to reproduce the AGN IR emission of bright quasars at different redshifts (Lyu et al. 2017), it is of considerable interest to explore whether they also apply to relatively low-luminosity AGNs. The possible existence of low-optical-depth dust in the vicinity of the AGN nucleus, as outlined above, motivates us to develop a new library of reddened AGN templates. We take the Lyu et al. (2017) empirical quasar templates as givens for polar-dust-free AGNs and investigate the extent to which the addition of a low optical depth but extended dust component with reasonable assumptions for the dust grain properties and their large-scale distribution can yield IR SED shapes consistent with those observed. In Section 2, we introduce this model and validate it by fitting the detailed observations of NGC 3783, the archetypal example of a type-1 AGN with its mid-IR emission dominated by polar dust.

Although the extended dust distribution may have a range of morphologies, any mid-infrared emission by low-optical-depth dust should be roughly isotropic and hence detectable from any view angle. We therefore focus on whether the model trained for NGC 3783 can be generally applied to match the infrared SEDs of those AGNs where standard quasar templates fail, assuming the choice of intrinsic AGN template and the optical depth of the obscuration as the only free parameters for the SED shape. This analysis is carried out on 64 low- $z$ Seyfert nuclei with negligible mid-IR star formation contamination in Section 3. In addition, if the polar dust emission is a common phenomenon for all populations of moderate-luminosity AGNs, a consequence is that Seyfert-1 nuclei should be moderately obscured on a statistical basis. We build composite SEDs of Seyfert-1 SEDs and confirm this prediction.

We find that the deviations from the quasar-like SED templates can indeed be explained to first order by the combination of extinction and infrared emission by polar dust. In Section 4, we characterize the SED features of the polar dust emission and discuss the prevalence of polar dust in a sample of AGNs much larger than those that can currently be explored in any detail through mid-infrared interferometry. We also demonstrate that one single semi-empirical template can describe the influence on the AGN SED by the polar dust emission for most objects. A consistency check of the results from our SED analysis and those from morphology-based identification for AGN polar dust emission is also carried out.

Various AGN populations with peculiar SED features that cannot be easily matched by the classical AGN templates have been reported at high $z$. Some notable examples are extremely red quasars (ERQs) (Ross et al. 2015; Hamann et al. 2017), AGNs with mid-IR warm-excess emission (e.g., $\mathrm{Xu}$ et al. 2015), and the hot dust-obscured galaxies (hot DOGs; e.g., Eisenhardt et al. 2012; Wu et al. 2012). The success of our model at low $z$ encourages us to explore if these peculiar SED features can be explained in a similar way. These studies are presented in Section 5.

Section 6 provides a discussion of the implications of these results for interpreting the AGN IR emission, the relation between X-ray obscuration and polar dust extinction, and the AGN unification scheme. We propose a tentative picture of the different circumnuclear dusty environments among AGNs that lead to their diverse IR properties. Section 7 is a final summary.

We adopt the cosmology $\Omega_{m}=0.27, \Omega_{\Lambda}=0.73$ and $H_{0}=$ $71 \mathrm{~km} \mathrm{~s}^{-1} \mathrm{Mpc}^{-1}$ (Bennett et al. 2003) throughout this paper. We use the term type-1 to describe AGNs showing broad emission lines without distinguishing if the line of sight (LOS) is dust-obscured or not. Since the word obscured is frequently reserved to describe type-2 (or narrow-line) AGNs, we adopt the name reddened type-1 AGNs to denote the broad-line AGNs with some extinction along the LOS (aka the polar direction), even if the real reddening might be insignificant because of a very flat extinction curve (e.g., Gaskell et al. 2004). Lastly, the word unobscured means no extinction, neither from the torus nor from any extended dust distribution, along the LOS to the central engine. 


\section{A Semi-empirical SED Model for Reddened Type-1 AGNs}

We introduce a relatively simple framework to produce a library of reddened type-1 AGN templates, which will be used to fit the SEDs of Seyfert-1 nuclei in Section 3. This model is based on two major assumptions:

1. Seyfert nuclei have a circumnuclear optically thick torus whose SED variations from a face-on viewpoint can be described by the intrinsic AGN templates of unobscured quasars;

2. Besides the torus, there could exist an extended dust component with some power-law density profile that is dominated by large dust grains heated by the AGN.

We describe the motivations as well as the details of these assumptions in Sections 2.1 and 2.2. The model and its behavior are presented in Section 2.3. In Section 2.4, we test our approach by fitting the observations of NGC 3783 .

\subsection{Accretion Disk and Dusty Torus}

The continuum SED of an AGN is contributed mostly by the UV-optical emission from the accretion disk around the $\mathrm{BH}$ and the near-IR to mid-IR emission emerges from the surrounding dusty structures. To reduce the uncertainties, we adopt well-tested extinction-free empirical templates to represent this AGN intrinsic emission.

The UV to mid-IR SEDs of most luminous type-1 quasars are well described by the Elvis et al. (1994)-like AGN template, regardless of the redshift (e.g., see discussion in Lyu et al. 2017). An AGN-heated dusty structure in type-1 quasars is revealed by the broad IR emission bump at $\lambda \sim 1.3-40 \mu \mathrm{m}$. In addition, broad emission lines have been detected in the optical polarized spectra of type-2 quasars (Zakamska et al. 2005). Under the precepts of AGN unification (Antonucci 1993; Urry \& Padovani 1995), these observations support the existence of some equatorial optically thick dusty structures, which cause the nuclear photons to preferentially escape along the polar direction.

As demonstrated by Lyu et al. (2017), the diversity of intrinsic IR emission among type-1 quasars at $z \sim 0-6$ can be characterized by three distinct templates derived for (1) normal AGNs, (2) WDD AGNs, and (3) HDD AGNs. These templates are unlikely to be affected significantly by dust extinction since their derivations are based on the study of optically blue quasars that are not obscured. In other words, there should be no significant dust distribution along the polar direction. We suggest these AGN templates describe the emission from the unobscured accretion disk plus a face-on view of the dusty torus. $^{1}$

At $\lambda<0.1 \mu \mathrm{m}$, current observations do not give good constraints. Following Stalevski et al. (2016), we assume a broken power law, where

$$
\nu F_{\nu} \propto \begin{cases}\lambda^{0} & 0.01 \mu \mathrm{m}<\lambda<0.1 \mu \mathrm{m} \\ \lambda^{1.2} & 0.001 \mu \mathrm{m}<\lambda<0.01 \mu \mathrm{m} .\end{cases}
$$

Nevertheless, our study will not be influenced by the assumed X-ray to UV SED shape. In fact, considering the likely

\footnotetext{
1 We use the word torus to describe the polar-dust-free obscuration structures as in optically blue quasars and do not make assumptions on the geometry or boundaries.
}

dominance of large dust grains along the face-on direction (see Section 2.2.1), the extinction at these wavelengths is small and will not contribute to the IR SEDs.

\subsection{Extended Polar Dust Component}

Besides the torus component characterized by the intrinsic AGN templates discussed above, we introduce another dust component to provide relatively low-level obscuration for the nucleus as well as the additional IR emission from the absorbed energy.

We suggest that the dust size distribution in this component is dominated by very large particles, which will be characterized by grain size cutoffs, $a_{\max }$ and $a_{\min }$. In real situations, different grain species sublimate at different temperatures and could have a broad range of dust sublimation zones. However, we find that the calculated SEDs do not change significantly between $T_{\text {sub }}=2000$ and $T_{\text {sub }}=1500$, indicating that the introduction of separate values appropriate for carbon and silicates would not change our results. For simplicity, we adopt the same dust sublimation temperature $T_{\text {sub }}$ for all the grain compositions.

For the large-scale structure, we assume a density profile parameterized as a power law in radius with slope $\alpha$ :

$$
\rho(r) \propto r^{-\alpha}, \quad r_{\text {in }}<r<r_{\text {out }},
$$

where the inner radius $r_{\text {in }}$ is equal to the dust sublimation radius $R_{\text {sub }}$ set by $T_{\text {sub }}$ as well as the light source luminosity, and the outer radius $r_{\text {out }}$ is a free parameter. We introduce another parameter, the outer-to-inner radius ratio $Y=r_{\text {out }} / r_{\text {in }}$, to describe $r_{\text {out }}$. Given the nature of this model, the geometry will not influence the dust emission SED so that there is no need to introduce more free parameters.

In the following, we outline the motivations behind these configurations.

\subsubsection{Grain Properties}

There are strong reasons to suggest that the classical dust properties are altered by the harsh environment of the direct exposure to an AGN. As suggested by Aitken \& Roche (1985), small grains $\left(a \sim 10^{-3} \mu \mathrm{m}\right)$ can be easily destroyed out to several hundred parsecs in a typical Seyfert-1 nucleus, on a timescale of less than a few years. Physically, the majority of dust destruction mechanisms around an AGN are relatively less significant for large dust grains (Laor \& Draine 1993). For example, large grains are expected to exist close to the torus inner part since they have smaller $R_{\text {sub }}$ than small grains. The torus itself can be dynamically unstable and material exchanges with the surrounding environments through various mechanisms are expected from simulations (e.g., Hopkins et al. 2012). Baskin \& Laor (2018) have analyzed the effects of sublimation on both carbon and silicate grains near an AGN. They found that only large $(a>0.1 \mu \mathrm{m})$ carbon grains can survive at the outer edge of the broad-line region out to about 20 times this radius. They also concluded that the silicate grain size distribution will be skewed toward large sizes to significantly greater distances.

Conditions for grain growth may exist in the circumnuclear tori, where the densities are high and grains are shielded from the X-ray and UV output of the central engine (e.g., Maiolino et al. 2001a). As suggested by, e.g., Hönig et al. (2012, 2013), some dust in the torus can be uplifted into the polar direction by 
AGN winds. It may also be possible for these large dust grains to form in situ. Elvis et al. (2002) suggested that dust can form in AGN-driven winds where conditions are similar to those in the winds of late-type stars. By these mechanisms, grains in the 0.1-1 $\mu \mathrm{m}$ range are plausible (Höfner 2008). However, determining observational constraints on the grain sizes around an AGN can be quite difficult. This can be seen from the diverse AGN UV-optical extinction curves reported that range from steeply rising SMC-like laws (e.g., Hall et al. 2002; Richards et al. 2003) to flat or gray laws (e.g., Czerny et al. 2004; Gaskell et al. 2004; Gaskell \& Benker 2007). As argued by, e.g., Baskin \& Laor (2018), it is possible that the grain properties depend on the observing angle.

Nonetheless, despite various uncertainties, there are observational indications of relatively large dust grains around AGNs, including (1) the lower ratios $A_{\mathrm{V}} / N_{H}$ and $E(B-V) / N_{H}$ in intermediate-type Seyfert galaxies (Maiolino et al. 2001b, 2001a; but see Weingartner \& Murray 2002); (2) the lower ratios between $A_{\mathrm{V}}$ and the mid-IR silicate absorption strength, $\Delta \tau_{9.7}$, in type-2 AGNs (Lyu et al. 2014; Shao et al. 2017); (3) successful fittings of the silicate emission profile in quasars and Seyfert galaxies with micron-sized grain models (Xie et al. 2017); and (4) the smaller observed torus inner radius from near-IR interferometry of nearby Seyfert nuclei compared with the expectations for classical dust grains (e.g.,Kishimoto et al. 2007, 2009; Burtscher et al. 2013; Hönig et al. 2013; but see Kawaguchi \& Mori 2010).

As shown in Appendix A, for classical interstellar medium (ISM) properties at low optical thickness, the mid-IR silicate emission feature at $\lambda \sim 10 \mu \mathrm{m}$ would be very prominent with a sharp peak (see also, e.g., Fritz et al. 2006; Nenkova et al. 2008a) that is not commonly seen among Seyfert nuclei (Hao et al. 2007). Instead, the lack of such detections could be an expected consequence of large grains. For example, dust grains with size $a \gtrsim 0.3 \mu \mathrm{m}$ would reduce the strength of silicate features effectively (Laor \& Draine 1993).

Due to the difficulties for setting direct constraints on the dust properties around the AGN, we minimize departures from standard ISM grain models and assume only the grain sizes, as characterized by the grain size cuts, $a_{\max }$ and $a_{\min }$, are altered in the vicinity of an AGN.

\subsubsection{Large-scale Geometry}

Currently we do not have strong observational constraints about the geometry of the dust responsible for the low-level obscuration in type-1 AGNs. Nevertheless, AGN outflows (e.g., Crenshaw et al. 2003; Piconcelli et al. 2005) could be a natural mechanism to distribute the dust around the nucleus. Physically, it has been found that the radiation pressure on resonant absorption lines alone cannot explain the outflow rates. The radiation feedback on dust within the clouds could be an effective mechanism (e.g., Roth et al. 2012). Based on a study of 3000 type-1 AGNs, Zhang et al. (2013) found that the relative strength of the mid-IR to the optical flux of these objects is correlated with the strength of outflows.

Several teams have tried to explore the origin of polar dust, showing the outflow scenario is a promising solution. For example, Hönig et al. $(2012$, 2013) proposed that the dusty outflows could be launched from the surface of the inner torus and the Hönig \& Kishimoto (2017) model motivated by this picture successfully explained the behavior of NGC 3783, a Seyfert-1 nucleus with a firm detection of polar dust emission.
From an analysis of high-spatial mid-IR images of 149 nearby Seyfert galaxies, Asmus et al. (2016) found that elongated polar dust emission is cospatial with the direction of AGN outflows for 18 objects.

Little is known about the exact density profile of the gas outflows. As a result, analytic analyses of self-similar solutions are typically pursued. We introduce a power-law density profile, $\rho(r) \propto r^{-\alpha}$, to approximate the real situations.

Physically, we do not expect the outflow solution retains the memory of initial conditions on large scales. As suggested by Faucher-Giguère \& Quataert (2012), to reach a finite-free expansion radius, the gas density profile should have profiles with $\alpha \lesssim 2$. Observationally, various values of $\alpha$ have been derived for materials in the outflows. For example, Behar (2009) derived $\alpha \sim 1.0-1.3$ for five nearby Seyfert nuclei by analyzing the X-ray absorption spectra. Feruglio et al. (2015) suggested an $r^{-2}$ profile for the ultra-luminous IR galaxy Mrk 231. Revalski et al. (2018) derived the electron density of the NLRs in the Seyfert-2 nucleus Mrk 573, finding $n_{e} \propto r^{-0.4}-r^{-0.6}$. Additionally, a constant density absorber $(\alpha=0)$ is quite unlikely the real case. Assuming that the dust and gas are well mixed with a constant dust-to-gas ratio, we suggest the dust density profile should satisfy $0<\alpha \lesssim 2$.

Based on mid-IR interferometry observations, AGN polar dust emission is found to be elongated (e.g., Hönig et al. 2012, 2013; Tristram et al. 2014; López-Gonzaga et al. 2016) and possibly distributed along the edges of the ionization cone (Stalevski et al. 2017). In our model, however, the likely uneven distribution of the polar dust component will not influence its IR emission SED. This is a direct consequence of optically thin dust emission in the IR, especially for large grains (e.g., Laor \& Draine 1993; Ivezic \& Elitzur 1997).

The modest levels of face-on extinction in type-1 AGNs correspond to a small value of $\tau_{\mathrm{V}}$. Since the dust opacity is a strong function of wavelength that decreases rapidly toward the infrared, the extinction for such IR-reprocessed dust emission is likely to be close to zero. In other words, the IR emission of any dusty structures with a low $\tau_{\mathrm{V}}$ is highly transparent: the emission from a single geometry element at some given location, where the included dust grains can be considered in local thermodynamic equilibrium (LTE), would share the same SED from different viewing angles and this SED would transit through other surrounding dusty structures without any notable changes. Consequently, the total integrated IR SED can be described as a summation of the dust emission from individual LTE geometry elements at all possible locations.

At the same distance $r$, the temperature of each LTE element would be the same as is the $\operatorname{SED}, B_{\lambda}(r)$. The total emission from all the dust at the same distance is linearly scaled by the total numbers of LTE elements at the corresponding radius, $\rho(r)$, and has little to do with their possible uneven distribution. The total integrated SED, $F_{\lambda}$, can be approximated by adding the contributions of all the elements at various radii, or

$$
F_{\lambda} \simeq \int_{r_{\text {in }}}^{r_{\text {out }}} \rho(r) B_{\lambda}(r) d r .
$$

Thus, if the average radial profile, $\rho(r)$, is similar, the dust distribution at small scales, whether it is smooth, clumpy or filamentary, will not influence the SED.

As long as the integrated optical depth, $\tau_{\mathrm{V}}$, is low, the effects of asymmetries in the dust large-scale structure and/or illumination along the radial directions would proportionally 


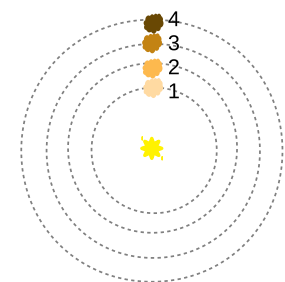

A1

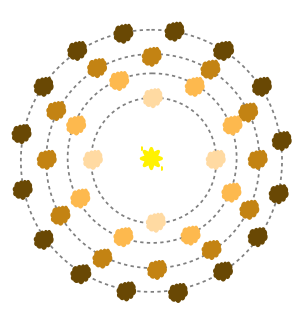

B1
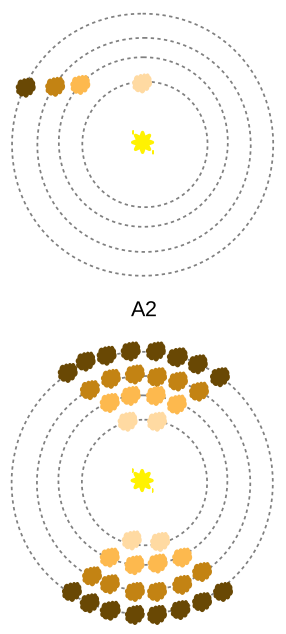

B2
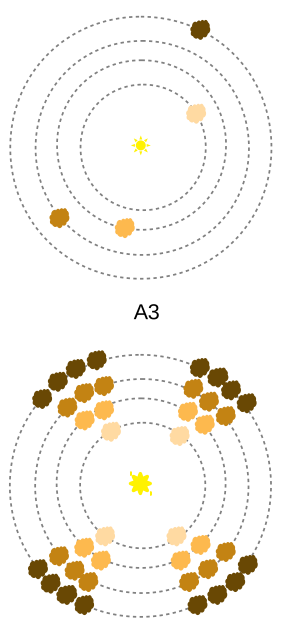

B3
Figure 1. Illustrations of the optically thin IR emission for different geometry configurations. Imagine that the dust structures are composed of similar small clouds at four different radii, $r_{1}, r_{2}, r_{3}$, and $r_{4}$, to the light source (the central yellow star in each panel). The total emission of all the clouds at a given $r_{i}$ would simply be $N_{i} F_{i}$, where $N_{I}$ is the number of clouds and $F_{i}=F_{i}(\nu)$ is the emission SED of one single cloud. Since the dust emission is assumed to be optically thin, the integrated emission of all the dust clouds with the geometries described in panels A1, A2, and A3 will be the same, $F_{\text {tot, } \mathrm{A}}=F_{1}+F_{2}+F_{3}+F_{4}$. Similarly, since the number of clouds, $N_{i}$, in each radius, $r_{i}$, is the same, the integrated SEDs in panels $\mathrm{B} 1, \mathrm{~B} 2$, and $\mathrm{B} 3$ are also identical for optically thin emission, and $F_{\text {tot, B }}=N_{1} F_{1}+N_{2} F_{2}+N_{3} F_{3}+$ $N_{4} F_{4}=2 F_{1}+4 F_{2}+6 F_{3}+8 F_{4}$, which is not dependent on how the clouds are distributed.

change $\rho(r)$, only resulting in a scaling down of the output polar dust emission. Note that $\tau_{\mathrm{V}}$ is linearly scaled with $\rho(r)$ by

$$
\tau_{\mathrm{V}}=\int_{r_{\text {in }}}^{r_{\text {out }}} \rho(r) C_{\mathrm{ext}, \mathrm{V}} d r=C_{\mathrm{ext}, \mathrm{V}} \int_{r_{\mathrm{in}}}^{r_{\mathrm{out}}} \rho(r) d r
$$

where $C_{\text {ext, }} \mathrm{V}$ is the optical V-band extinction of all the grains within a single LTE element. Thus, these geometry effects can be modeled by simply changing $\tau_{\mathrm{V}}$. Figure 1 provides a simple illustration. In Appendix A, we provide some simple demonstrations of the lack of influence of the dust geometry on the derived SED with three-dimensional dust radiative transfer simulations. Although the distribution of polar dust realistically could be highly complicated, for the purpose of determining the resulting SED it is equivalent to assume spherical symmetry.

In summary, when the $\tau_{\mathrm{V}}$ of the extended dust component is not very high, its IR SED shape would be only dependent on the radial density profile. Since the nuclear dust morphology cannot be constrained for most AGNs, we will not introduce detailed geometry to match the very few observations.

\subsection{Reddened Type-1 SEDs from the Model}

We use the latest version of the radiative transfer code DUSTY (Ivezic et al. 2017) ${ }^{2}$ to obscure the three AGN templates presented in Lyu et al. (2017). This code assumes a spherical symmetry and solves the one-dimensional radiation transfer equations as described in Ivezic \& Elitzur (1997). Although a realistic model would place the emitting clouds preferentially in the polar direction, we have just shown that the output spectrum for the optically thin case would be identical.

\footnotetext{
2 Accessible at https://github.com/ivezic/dusty.
}

Table 1

DUSTY Model Setup

\begin{tabular}{lcl}
\hline \hline Parameter & Label & Value \\
\hline$r_{\text {in temperature }}$ & $T_{\text {in }}$ & $2000 \mathrm{~K}, 1500^{*} \mathrm{~K}, 1000 \mathrm{~K}$ \\
Density profile & $\alpha$ & $0,0.5^{*}, 1,1.5,2$ \\
Outer-to-inner radius & $Y$ & $50,500^{*}, 5000$ \\
Silicate:graphite mixture & & $0: 1,0.53: 0.47^{*}, 1: 0$ \\
Maximum grain size & $a_{\max }$ & $0.25,2.5,10^{*}, 100$ \\
Minimum grain size & $a_{\min }$ & $0.005,0.01,0.05^{*}, 0.1$ \\
\hline Input radiation SED & & Norm, WDD* HDD \\
Optical depth & $\tau_{\mathrm{V}}$ & $0-10$ with a step of 0.25 \\
\hline
\end{tabular}

Note. We use ${ }^{*}$ to indicate the reference parameters that were adopted to demonstrate the influence of the output SEDs in Figure 2.

For a centrally heated spherical density distribution, the program needs the SED of the radiation source, a density profile of the dust distribution and information regarding the boundaries, the dust properties (chemical composition, grain size distribution and the sublimation temperature), and a range of required optical depths at some specific wavelength.

We calculate three sets of reddened templates separately for normal, HDD, and WDD AGNs. The model parameters are summarized in Table 1. We adopted optical properties for graphite and silicate grains from Draine \& Lee (1984) with the standard Mathis-Rumpl-Nordsieck power-law grain size distribution distribution $d n / d a \propto a^{-3.5}$ (Mathis et al. 1977), but left the boundaries, $a_{\max }$ and $a_{\min }$, to be varied.

Figure 2 presents the reddened WDD AGN SEDs as a function of the optical extinction level $\tau_{\mathrm{V}}$ for different values of model parameters. It is interesting that for large dust grains, the dust-reprocessed SEDs are not sensitive to the mixtures of silicates and graphites. For a given $\tau_{\mathrm{V}}$, the amount of UVoptical reddening is only sensitive to the smallest grain size $a_{\text {min }}$ and has little to do with the dust geometry configurations. The strength of the mid-IR silicate emission feature is sensitive to the maximum grain size $a_{\max }$ and the geometry parameters $Y$ and $\alpha$. The relative strength of the hot dust emission is mainly determined by the compactness of the dust distribution $(\alpha, Y)$. Finally, the shape of the broad-band IR continuum is mainly determined by the assumed geometry (as well as the input intrinsic SED).

Since the dust temperature at the inner radius $\left(T_{\text {in }}=T_{\text {sub }}\right)$ and relative fraction between silicate and graphite grains have limited effects on the reddened SEDs, we decide to adopt $T_{\text {in }}=1500 \mathrm{~K}$, and a normal 0.53:0.47 mixture of silicate and graphite grains.

\subsection{Model Validation with Observations of NGC 3783}

Our proposed simple model differs substantially from the approach of Hönig \& Kishimoto (2017), who fitted the SED and interferometric data for NGC 3783 with a specific system of very optically thick $\left(\tau_{\mathrm{V}}=50\right)$ small clouds. We show here that our model can fit the SED of this object equally well.

We adopted the X-ray to mid-IR subarcsecond high-spatialresolution (HSR) nuclear photometry SED of NGC 3783 presented in Prieto et al. (2010). To improve the mid-IR continuum constraints, we collected its $2.5-5 \mu \mathrm{m}$ AKARI spectrum (Kim et al. 2015) and 5.5-38 $\mu \mathrm{m}$ Spitzer/IRS spectrum (Lebouteiller et al. 2011). Since the spectral flux is consistent with the HSR photometry at corresponding 

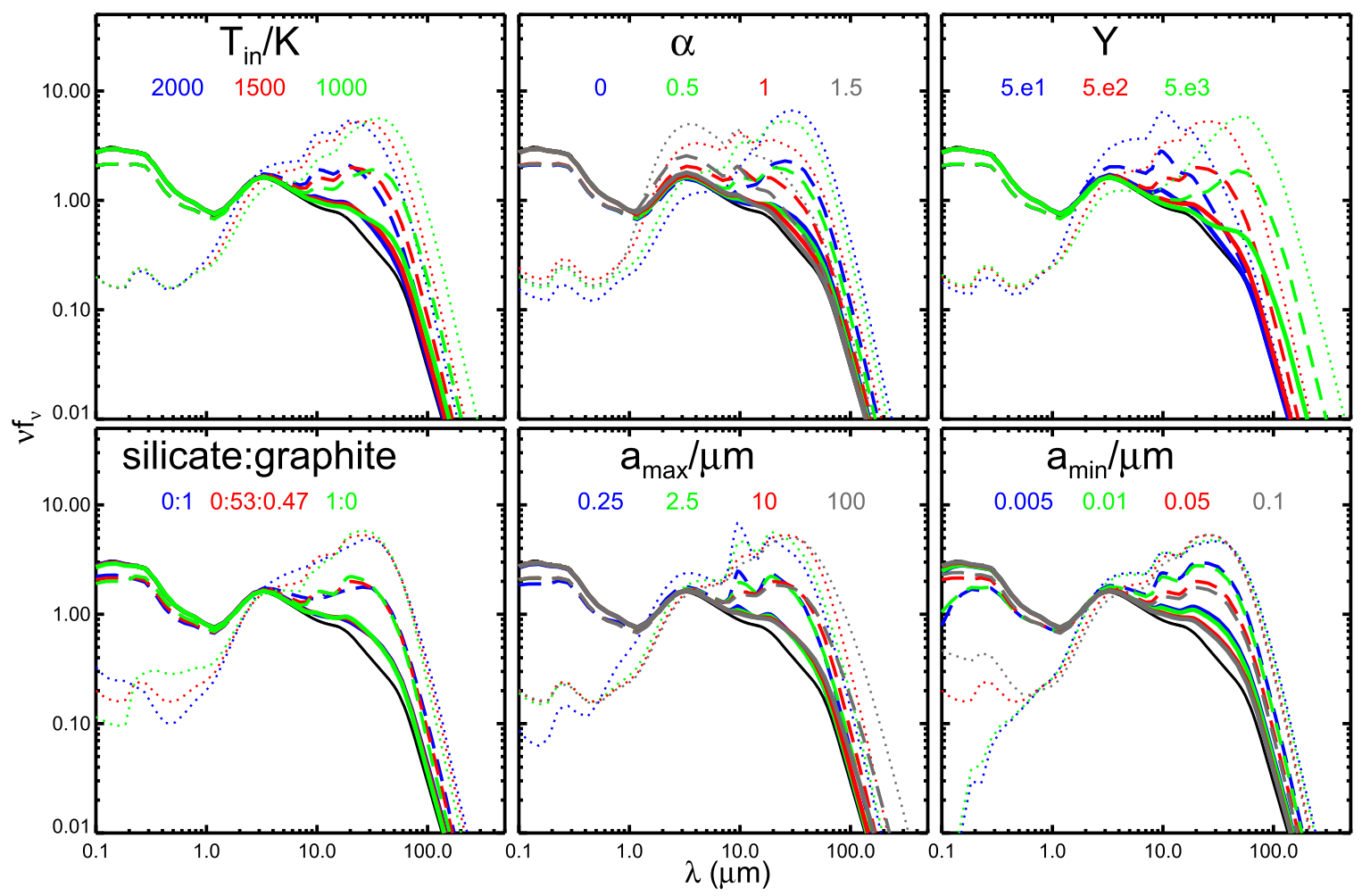

Figure 2. The influence of model parameters on the output SEDs. For each model setup, we computed the output SEDs with different levels of optical extinction $\tau_{\mathrm{V}}=0.1$ (solid lines), 0.63 (dashed lines), and 3.98 (dotted lines).

wavelengths (the Spitzer/IRS flux is also identical to the spectrum obtained through a 0 "! $75 \times 0$ ". 53 aperture; see Figure 3 in Hönig et al. 2013) and there is no obvious star formation feature, we believe these data apply to the AGN emission. To gauge the far-IR emission strength from the nucleus, we used the $1 \mathrm{kpc}$ aperture Herschel 70 and $100 \mu \mathrm{m}$ photometry of NGC 3783 from García-González et al. (2016). For the foreground extinction by the Milky Way, we obtained $E(B-V)=0.10$ from the Schlegel, Finkbeiner \& Davis (SFD) dust map (Schlegel et al. 1998; Schlafly \& Finkbeiner 2011) and used the Fitzpatrick (1999) extinction law to correct the UV-optical data.

For NGC 3783, the torus component is believed to dominate the near-IR emission but not the mid-IR (Hönig et al. 2013). After comparing the observed SED of this object with the Lyu et al. (2017) intrinsic AGN templates, we suggest its torus emission should be WDD-like. The 0.1-30 $\mu \mathrm{m}$ SED of NGC 3783 is then fitted by the reddened WDD AGN template with parameter ranges for the polar dust component in Table 1 . We use the Markov chain Monte Carlo (MCMC) algorithms described by Johnson et al. (2013) to sample the large parameter space and find the most likely parameter combination. The values of the best-fit parameters and their 1- $\sigma$ uncertainties (68\% confidence levels) are summarized in Table 2. The large fitted parameter errors suggest a strong degeneracy. After considering the possible contamination by optical emission lines and the fact that the far-IR photometry provides an upper limit to the AGN-heated dust emission, we finally decide to adopt $\rho(r) \propto r^{-0.5}, Y=500$ and $T_{\text {in }}=1500$ $\mathrm{K}$, and grain size cutoffs at $a_{\min }=0.04 \mu \mathrm{m}$ and $a_{\max }=$ $10 \mu \mathrm{m}$. Most of these values are chosen near the midpoint of the fitted ranges for illustration.
Table 2

Suggested Model Parameters for NGC 3783

\begin{tabular}{lccc}
\hline \hline Parameter & Label & Adopted Value & MCMC output \\
\hline$r_{\text {in }}$ temperature & $T_{\text {in }}$ & $\mathbf{1 5 0 0 ~ K}$ & $\ldots$ \\
Density profile & $\alpha$ & 0.50 & $0.47_{-0.44}^{+0.50}$ \\
Outer-to-inner radius & $Y$ & 500 & $475_{-353}^{+513}$ \\
Silicate:graphite mixture & $\ldots$ & $\mathbf{0 . 5 3 : 0 . 4 7}$ & $\ldots$ \\
Maximum grain size & $a_{\max }$ & 10 & $20_{-18}^{+79}$ \\
Minimum grain size & $a_{\min }$ & 0.04 & $0.05_{-0.04}^{+0.05}$ \\
\hline Input radiation SED & $\ldots$ & $\mathbf{W D D}$ & $\ldots$ \\
Optical depth & $\tau_{\mathrm{V}}$ & 1.4 & $1.44_{-0.60}^{+0.55}$ \\
\hline
\end{tabular}

Note. We use boldface to indicate assumed parameter values that do not go to MCMC parameter space sampling.

As shown in the left panel of Figure 3, the UV to far-IR nuclear SED of NGC 3783 is reasonably matched by the WDD AGN template obscured by the suggested polar dust component with an optical depth $\tau_{\mathrm{V}}=1.4$. In the right panel, we show the relative contributions of the attenuated and scattered WDD emission, as well as the polar dust emission in the best model. Besides the near-IR, the torus emission SED of the WDD AGN is not modified by the polar dust obscuration, so we can linearly separate the torus emission and the polar dust emission. At $\sim 10 \mu \mathrm{m}$, the optically thin component would contribute about $75 \%$ of the total emission, which is in good agreement with the polar dust emission strength constrained by interferometry (Hönig et al. 2013; López-Gonzaga et al. 2016). Since the highly optically thick torus can block the accretion disk emission along the equatorial direction and the dust along the polar direction is likely to be preferentially heated, some elongated emission from the regions responsible for the 


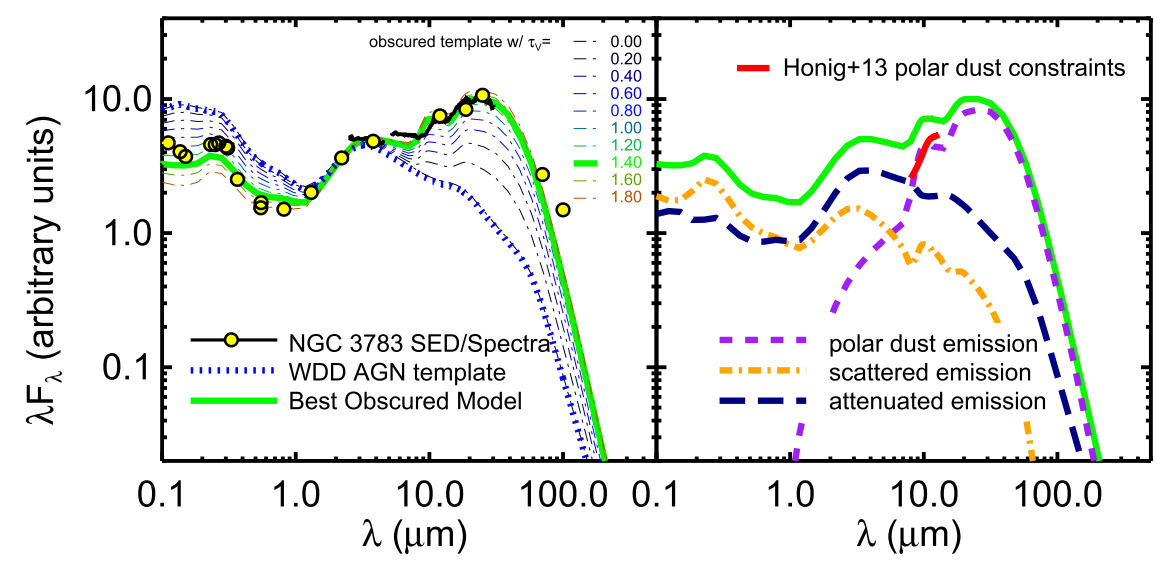

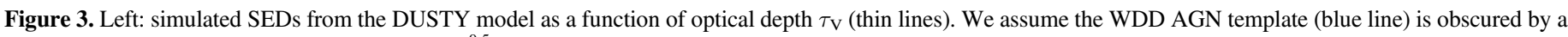

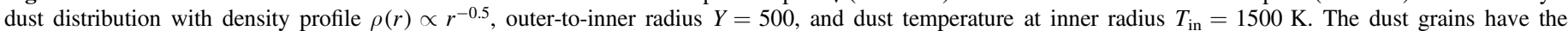

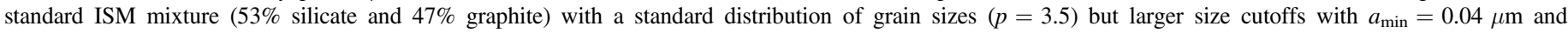

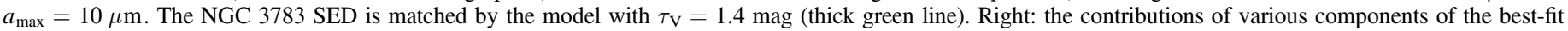
model as a function of wavelength. We also plot the 8-13 $\mu \mathrm{m}$ polar dust emission strength constrained by Hönig et al. (2013) for comparison.

low-level obscuration could be observable if the system has an appropriate viewing angle.

Although our goal does not include reproducing any detailed dust morphology, this model should yield similar sizes of the dusty structures to the observations. From fitting the mid-IR interferometry data of NGC 3783, Hönig et al. (2013) reported that the nuclear dust is distributed over 20-70 $r_{\text {in }}$, where $r_{\text {in }} \sim 0.06 \mathrm{pc}$ is derived from near-IR reverberations. The model geometry $r_{\text {out }} / r_{\text {in }} \sim 500$ suggested from our SED fitting is much larger than this observation. However, at a given wavelength, we only observe dust with a narrow range of dust temperatures; the outer part of the extended dust component would be too cold to observe in the mid-IR. Based on the radial profile of dust temperature in our model, the observed geometry seen at $8-13 \mu \mathrm{m}$ would have a size of $20-40 r_{\text {in }}$, which in fact qualitatively agrees with the Hönig et al. (2013) observations (see more discussion in Section 4.2).

\subsection{The Degeneracy of Models Constrained Only by the $A G N$ SED}

The degeneracies of SED fitting - as shown here, equally good results from the Hönig \& Kishimoto (2017) optically thick clumpy dust model and our simple low-optical-depth obscuration model based on empirical AGN templatesindicate that the same observations can be explained through rather different descriptions about the AGN circumnuclear extended dust. In general, we lack any detailed structural information in the mid-IR for most known AGNs. Consequently, additional parameters to characterize the geometry of this structure cannot be realistically constrained. As explained in Section 2.2.2, at low optical depth, the behavior of polar dust emission should not be sensitive to the shape of its large-scale distribution, nor the observing angle. Thus, our reddened AGN SED model is consistent with the possible diverse polar dust morphologies.

\section{Reproducing the SEDs of Low- $z$ Seyfert Nuclei}

\subsection{Sample and Data}

In view of the success in reproducing the observations of NGC 3783, we will test if a similar model works for other Seyfert nuclei as well. To reduce the ambiguity in interpreting the SED, we have searched the literature and archives to find low- $z$ Seyfert- 1 nuclei where the AGN mid-IR emission can be isolated from the host star formation contamination or the contribution from the latter can be safely ignored. The details can be found in Appendix B. Below is a brief summary.

We first compiled AGNs with HSR mid-IR SED constraints. About half of them are Seyfert-1-1.5 nuclei from Asmus et al. (2014); we required that the ground-based $12 \mu \mathrm{m}$ flux measurements be consistent with the much larger beam Widefield Infrared Survey Explorer (WISE) W3 band observations. We also included some very well-studied Seyfert-1 nuclei and all the type-1 objects with mid-IR interferometry observations. Due to these selections, the near-IR stellar contamination is not very significant in this HSR sample. In total, there are 32 nuclei selected. These objects span a redshift range of 0.002-0.16, with a median at $z=0.02$. IR photometry from the literature and surveys like the Two Micron All Sky Survey (2MASS), the UKIRT Infrared Deep Sky Surve (UKIDSS), and WISE were collected to define the SEDs.

In addition, we looked for optically selected broad-line AGNs from the Sloan Digital Sky Survey (SDSS; York et al. 2000) that have Spitzer/IRS spectra allowing isolation of the nuclear emission in the mid-IR. The homogeneous datasets of this sample enable the derivation of composite SEDs that minimize the effects of different observing angles and variability, and can be used to test the complete range of reddened AGN SEDs predicted from our model. For this SDSS/Spitzer sample, we ended up with 33 type-1 AGNs with a redshift range of $0.008-0.20$, with the median at $z=0.06$. Except for NGC 4235, all of them are new objects with no duplication found in the HSR sample. Archival and literature X-ray, UV, optical and IR data from e.g., XMM-Newton, Chandra, Galaxy Evolution Explorer (GALEX), Hubble, 2MASS, WISE, Spitzer, AKARI, Herschel, and other groundbased optical and IR facilities are collected for these objects with a careful selection of photometry apertures if possible.

To get a rough idea of the influence of variabilities on the IR SEDs of these objects, we determined their mid-IR variability amplitudes in the WISE $\mathrm{W} 1$ and $\mathrm{W} 2$ bands using the archival data from the WISE (Wright et al. 2010) and the Near-Earth Object WISE Reactivation mission (NEOWISE-R; Mainzer et al. 2014) missions, covering the time period of 2010-2017. 
Table 3

List of the Low-redshift Seyfert-1 Nuclei

\begin{tabular}{|c|c|c|c|c|c|c|c|c|c|c|c|c|c|}
\hline \multirow{2}{*}{$\begin{array}{l}\# \\
\text { (1) }\end{array}$} & \multirow{2}{*}{$\begin{array}{c}\text { Name } \\
\text { (2) }\end{array}$} & \multirow{2}{*}{$\begin{array}{l}z \\
(3)\end{array}$} & \multicolumn{3}{|c|}{ References for the IR SED Data } & \multirow{2}{*}{$\begin{array}{c}F_{\mathrm{g}} / F_{\mathrm{s}} \\
(7)\end{array}$} & \multirow{2}{*}{$\begin{array}{c}f_{\mathrm{MIR}, \mathrm{SF}} \\
\% \\
(8)\end{array}$} & \multirow{2}{*}{$\begin{array}{c}\mathrm{EW}_{\mathrm{PAH}} \\
\mu \mathrm{m} \\
(9)\end{array}$} & \multirow{2}{*}{$\begin{array}{l}\Delta \mathrm{W} 1 \\
\mathrm{mag} . \\
(10)\end{array}$} & \multirow{2}{*}{$\begin{array}{l}\Delta \mathrm{W} 2 \\
\mathrm{mag} . \\
(11)\end{array}$} & \multirow{2}{*}{$\begin{array}{c}\text { AGN type } \\
\text { (12) }\end{array}$} & \multirow{2}{*}{$\begin{array}{c}\tau_{\mathrm{V}, \text { ext. }} \\
\text { (13) }\end{array}$} & \multirow{2}{*}{$\begin{array}{c}f_{\mathrm{pol}, 10 \mu \mathrm{m}} \\
\text { (14) }\end{array}$} \\
\hline & & & $\begin{array}{c}(\lambda \sim 1-2.5 \mu \mathrm{m}) \\
\text { (4) }\end{array}$ & $(\lambda \sim 2.5-40 \mu \mathrm{m})$ & $\begin{array}{c}(\lambda>40 \mu \mathrm{m}) \\
(6)\end{array}$ & & & & & & & & \\
\hline \multicolumn{14}{|c|}{ HSR AGN Sample } \\
\hline 1 & PKS 1417-19 & 0.1195 & $2 \mathrm{M}$ & $\mathrm{W}$ & $\ldots$ & 0.93 & $\ldots$ & $\ldots$ & 0.31 & 0.26 & WDD & 1.00 & 0.65 \\
\hline 2 & ESO 141-55 & 0.0371 & $2 \mathrm{M}$ & $\mathrm{W}$ & $1,2,3$ & 0.94 & $\ldots$ & $\ldots$ & 0.26 & 0.23 & WDD & 0.25 & 0.30 \\
\hline 3 & Mrk 509 & 0.0344 & $2 \mathrm{M}$ & W & 2,3 & 0.99 & $\ldots$ & $\ldots$ & 0.25 & 0.15 & WDD & 0.50 & 0.47 \\
\hline 4 & Mrk 1239 & 0.0199 & 5,6 & $3,4,8,9,10,11,12$ & 11 & 0.99 & $\ldots$ & $\ldots$ & 0.40 & 0.27 & Norm & 0.00 & 0.00 \\
\hline 5 & $3 \mathrm{C} 382$ & 0.0579 & $2 \mathrm{M}$ & $\mathrm{W}, 1$ & 4 & 1.02 & $\ldots$ & $\ldots$ & 0.27 & 0.23 & WDD & 0.00 & 0.00 \\
\hline 6 & IRAS 09149-6206 & 0.0573 & $2 \mathrm{M}$ & $\mathrm{W}, 2$ & 5 & 1.09 & $\ldots$ & $\ldots$ & 0.28 & 0.20 & WDD & 0.25 & 0.30 \\
\hline 7 & Ark 120 & 0.0327 & $2 \mathrm{M}$ & W & 2,3 & 1.13 & $\ldots$ & $\ldots$ & 0.38 & 0.37 & WDD & 0.00 & 0.00 \\
\hline 8 & Fairall 51 & 0.0142 & $2 \mathrm{M}$ & $\mathrm{W}$ & 2,3 & 1.13 & $\ldots$ & $\ldots$ & 0.16 & 0.13 & WDD & 0.50 & 0.47 \\
\hline 9 & IRAS $13349+2438$ & 0.1076 & $2 \mathrm{M}$ & W & 1 & 1.14 & $\ldots$ & $\ldots$ & 0.09 & 0.05 & Norm & $2.50^{\mathrm{a}}$ & $0.83^{\mathrm{a}}$ \\
\hline 10 & I Zw 1 & 0.0589 & $2 \mathrm{M}$ & $\mathrm{W}, 13$ & 12 & 1.16 & $\ldots$ & $\ldots$ & 0.29 & 0.20 & Norm & 0.50 & 0.33 \\
\hline 11 & H 0557-385 & 0.0339 & $2 \mathrm{M}$ & $\mathrm{W}, 1$ & 2 & 1.18 & $\ldots$ & $\ldots$ & 0.24 & 0.15 & WDD & 0.25 & 0.30 \\
\hline 12 & IC 4329A & 0.0161 & $2 \mathrm{M}$ & $\mathrm{W}, 1$ & 2,3 & 1.18 & $\ldots$ & $\ldots$ & 0.37 & 0.33 & WDD & 0.75 & 0.58 \\
\hline 13 & $3 \mathrm{C} 120$ & 0.0330 & $2 \mathrm{M}$ & W & 2,3 & 1.20 & $\ldots$ & $\ldots$ & 0.33 & 0.26 & WDD & 0.75 & 0.58 \\
\hline 14 & $3 C 390.3$ & 0.0561 & $2 \mathrm{M}$ & $\mathrm{W}, 1$ & 8 & 1.20 & $\ldots$ & $\ldots$ & 0.35 & 0.29 & WDD & 0.50 & 0.47 \\
\hline 15 & Pictor A & 0.0351 & $2 \mathrm{M}$ & W & 2,3 & 1.21 & $\ldots$ & $\ldots$ & 0.32 & 0.30 & WDD & 0.50 & 0.47 \\
\hline 16 & MR 2251-178 & 0.0640 & 1 & $\mathrm{~W}, 1,3$ & 9 & 1.21 & $\ldots$ & $\ldots$ & $? ?$ & $? ?$ & WDD & 0.25 & 0.30 \\
\hline 17 & $3 \mathrm{C} 227$ & 0.0858 & $2 \mathrm{M}$ & $\mathrm{w}, 3,6$ & 10 & 1.28 & $\ldots$ & $\ldots$ & 1.02 & 0.52 & WDD & 0.25 & 0.30 \\
\hline 18 & Mrk 1014 & 0.1631 & $2 \mathrm{M}$ & W & $11,12,13$ & 1.28 & $\ldots$ & $\ldots$ & 0.24 & 0.18 & Norm & 10.00 & 0.93 \\
\hline 19 & $3 \mathrm{C} 445$ & 0.0559 & $2 \mathrm{M}$ & W & 1 & 1.29 & $\ldots$ & $\ldots$ & 0.13 & 0.08 & Norm & 0.00 & 0.00 \\
\hline 20 & $3 \mathrm{C} 93$ & 0.3571 & $2 \mathrm{M}$ & W & $\ldots$ & 1.54 & $\ldots$ & $\ldots$ & 0.33 & 0.41 & WDD & 0.50 & 0.47 \\
\hline 21 & NGC 3783 & 0.0097 & P10 & $\mathrm{P} 10$ & $2,3,19$ & 1.03 & $\ldots$ & $\ldots$ & 1.91 & 2.37 & Norm & 1.00 & 0.51 \\
\hline 22 & NGC 4507 & 0.0118 & $2 \mathrm{M}$ & W & 2,3 & 1.15 & $\ldots$ & $\ldots$ & 0.16 & 0.18 & WDD & 1.75 & 0.78 \\
\hline 23 & ESO 323-77 & 0.0150 & $2 \mathrm{M}$ & $1,3,7$ & $2,3,19$ & 0.67 & $\ldots$ & $\ldots$ & 0.31 & 0.26 & WDD & 0.25 & 0.30 \\
\hline 24 & NGC 4151 & 0.0033 & AH13 & AH13 & 19 & 0.78 & $\ldots$ & $\ldots$ & 1.15 & 0.78 & Norm & 0.75 & 0.44 \\
\hline 25 & NGC 7469 & 0.0163 & $\mathrm{P} 10, \mathrm{AH} 13$ & $\mathrm{P} 10, \mathrm{AH} 13$ & 2,3 & 0.48 & $\cdots$ & $\ldots$ & 0.34 & 0.31 & Norm & 7.75 & 0.91 \\
\hline 26 & NGC 1566 & 0.0050 & P10 & P10 & & 0.36 & $\ldots$ & $\ldots$ & 0.11 & 0.32 & Norm & 9.50 & 0.93 \\
\hline 27 & NGC 4593 & 0.0090 & $2 \mathrm{M}$ & $\mathrm{W}, 3$ & 11 & 0.85 & $\ldots$ & $\ldots$ & 0.35 & 0.52 & WDD & 0.75 & 0.58 \\
\hline 28 & NGC 3227 & 0.0039 & F16 & F16 & 2,3 & 0.40 & $\ldots$ & $\ldots$ & 0.25 & 0.25 & HDD & 4.50 & 0.94 \\
\hline 29 & NGC 4235 & 0.0080 & AH13 & AH13 & 2,14 & 0.78 & $\ldots$ & $\ldots$ & 0.23 & 0.26 & Norm & 0.00 & 0.00 \\
\hline 30 & NGC 4015 & 0.0023 & $2 \mathrm{M}$ & W & 9 & $\ldots$ & $\ldots$ & $\ldots$ & 0.41 & 0.43 & Norm? & $1.75 ?$ & 0.66 ? \\
\hline 31 & Fairall 9 & 0.0470 & $2 \mathrm{M}$ & W & 2,3 & 1.11 & $\ldots$ & $\ldots$ & 0.09 & 0.04 & Norm & 0.00 & 0.00 \\
\hline 32 & NGC 3516 & 0.0088 & AH13 & AH13 & 2,3 & $\ldots$ & $\ldots$ & $\ldots$ & 0.66 & 0.79 & Norm & $1.00 ?$ & $0.51 ?$ \\
\hline \multicolumn{14}{|c|}{ SDSS/Spitzer Sample } \\
\hline 33 & Mrk 1393 & 0.0543 & $2 \mathrm{M}$ & $\mathrm{W}$ & 15 & $\ldots$ & 0.2 & 0.01 & 0.22 & 0.23 & Norm & 0.75 & 0.44 \\
\hline 34 & Mrk 506 & 0.0431 & $2 \mathrm{M}$ & W & $\ldots$ & $\ldots$ & 2.2 & 0.03 & $? ?$ & $? ?$ & WDD & 0.25 & 0.30 \\
\hline 35 & Mrk 926 & 0.0470 & $2 \mathrm{M}$ & $\mathrm{W}$ & 2,3 & $\ldots$ & 4.6 & 0.01 & 0.73 & 0.61 & Norm & 0.25 & 0.20 \\
\hline 36 & NGC 4074 & 0.0226 & $2 \mathrm{M}$ & $\mathrm{W}$ & 2,3 & $\ldots$ & 7.3 & 0.02 & $? ?$ & $? ?$ & Norm & 1.50 & 0.62 \\
\hline 37 & Mrk 1392 & 0.0359 & $2 \mathrm{M}$ & $\mathrm{W}$ & 2,3 & $\ldots$ & 1.9 & 0.01 & $? ?$ & $? ?$ & Norm & 0.75 & 0.44 \\
\hline 38 & [VV2006c] J020823.8-002000 & 0.0741 & $2 \mathrm{M}$ & $\mathrm{W}$ & $\ldots$ & $\ldots$ & 4.5 & 0.00 & 0.19 & 0.21 & Norm & 1.00 & 0.51 \\
\hline 39 & NGC 2484 & 0.0408 & $2 \mathrm{M}$ & $\mathrm{W}$ & $\ldots$ & $\ldots$ & 1.7 & 0.00 & 0.08 & 0.15 & Norm? & $10.00 ?$ & $0.93 ?$ \\
\hline 40 & 2MASX J14510879+2709272 & 0.0645 & $2 \mathrm{M}$ & W & 12 & $\ldots$ & 1.3 & 0.05 & $? ?$ & $? ?$ & Norm & 0.50 & 0.33 \\
\hline 41 & 2MASX J16164729+3716209 & 0.1518 & $2 \mathrm{M}$ & $\mathrm{W}$ & 16 & $\ldots$ & 0.0 & 0.03 & 0.03 & 0.08 & Norm & 10.00 & 0.93 \\
\hline 42 & NGC 863 & 0.0261 & $2 \mathrm{M}$ & W & 2,3 & 1.43 & 2.2 & 0.03 & 0.13 & 0.34 & Norm & 4.00 & 0.84 \\
\hline
\end{tabular}


Table 3

(Continued)

\begin{tabular}{|c|c|c|c|c|c|c|c|c|c|c|c|c|c|}
\hline \multirow{2}{*}{$\begin{array}{l}\# \\
\text { (1) }\end{array}$} & \multirow{2}{*}{$\begin{array}{c}\text { Name } \\
\text { (2) }\end{array}$} & \multirow{2}{*}{$\begin{array}{l}z \\
\text { (3) }\end{array}$} & \multicolumn{3}{|c|}{ References for the IR SED Data } & \multirow{2}{*}{$\begin{array}{c}F_{\mathrm{g}} / F_{\mathrm{s}} \\
(7)\end{array}$} & \multirow{2}{*}{$\begin{array}{c}f_{\mathrm{MIR}, \mathrm{SF}} \\
\% \\
(8)\end{array}$} & \multirow{2}{*}{$\begin{array}{c}\mathrm{EW}_{\mathrm{PAH}} \\
\mu \mathrm{m} \\
(9)\end{array}$} & \multirow{2}{*}{$\begin{array}{l}\Delta \mathrm{W} 1 \\
\text { mag. } \\
(10)\end{array}$} & \multirow{2}{*}{$\begin{array}{c}\Delta \mathrm{W} 2 \\
\mathrm{mag} . \\
(11)\end{array}$} & \multirow{2}{*}{$\begin{array}{c}\text { AGN type } \\
\text { (12) }\end{array}$} & \multirow{2}{*}{$\begin{array}{c}\tau_{\mathrm{V}, \text { ext. }} \\
(13)\end{array}$} & \multirow{2}{*}{$\begin{array}{c}f_{\mathrm{pol}, 10 \mu \mathrm{m}} \\
\quad(14)\end{array}$} \\
\hline & & & $\begin{array}{c}(\lambda \sim 1-2.5 \mu \mathrm{m}) \\
\text { (4) }\end{array}$ & $(\lambda \sim 2.5-40 \mu \mathrm{m})$ & $\begin{array}{c}(\lambda>40 \mu \mathrm{m}) \\
(6)\end{array}$ & & & & & & & & \\
\hline 43 & Mrk 1018 & 0.0430 & $2 \mathrm{M}$ & $\mathrm{W}$ & 2,3 & $\ldots$ & 4.4 & 0.00 & 0.77 & 1.11 & Norm & 0.00 & 0.00 \\
\hline 44 & $3 \mathrm{C} 15$ & 0.0735 & $2 \mathrm{M}$ & $\mathrm{W}$ & $\ldots$ & $\ldots$ & 1.2 & 0.06 & 0.13 & 0.20 & WDD & 0.25 & 0.30 \\
\hline 45 & 2dFGRS TGN254Z050 & 0.0888 & $2 \mathrm{M}$ & W & 16 & $\ldots$ & 0.0 & 0.04 & $? ?$ & $? ?$ & Norm & 10.00 & 0.93 \\
\hline 46 & Ton 730 & 0.0864 & $2 \mathrm{M}$ & $\mathrm{W}$ & 12 & $\ldots$ & 0.6 & 0.01 & $? ?$ & $? ?$ & HDD & 0.25 & 0.42 \\
\hline 47 & Mrk 110 & 0.0355 & $2 \mathrm{M}$ & $\mathrm{W}$ & 12 & $\ldots$ & 6.7 & 0.00 & $? ?$ & $? ?$ & Norm & 0.00 & 0.00 \\
\hline 48 & 2MASX J09191322+5527552 & 0.0489 & $2 \mathrm{M}$ & $\mathrm{W}$ & 2,3 & $\ldots$ & 6.1 & 0.00 & 0.22 & 0.20 & Norm & 1.00 & 0.51 \\
\hline 49 & 2MASX J14492067+4221013 & 0.1786 & $2 \mathrm{M}$ & $\mathrm{W}$ & 16 & $\ldots$ & 0.4 & 0.03 & 0.06 & 0.10 & Norm & $7.75^{\mathrm{a}}$ & $0.95^{\mathrm{a}}$ \\
\hline 50 & NGC 5252 & 0.0229 & $2 \mathrm{M}$ & $\mathrm{W}$ & 2,3 & $\ldots$ & 2.2 & 0.01 & 0.87 & 0.98 & WDD & 0.00 & 0.00 \\
\hline 51 & $3 \mathrm{C} 219$ & 0.1746 & $2 \mathrm{M}$ & $\mathrm{W}$ & $\ldots$ & $\ldots$ & 2.8 & 0.02 & 0.10 & 0.12 & WDD & 0.25 & 0.30 \\
\hline 52 & SDSS J095504.55+170556.3 & 0.1378 & $2 \mathrm{M}$ & $\mathrm{W}$ & 12 & $\ldots$ & 1.5 & 0.00 & 0.32 & 0.22 & Norm & 0.00 & 0.00 \\
\hline 53 & Mrk 176 & 0.0265 & $2 \mathrm{M}$ & $\mathrm{W}$ & 17 & $\ldots$ & 8.9 & 0.04 & 0.16 & 0.12 & Norm & 1.00 & 0.51 \\
\hline 54 & 2MASX J14054117+4026326 & 0.0806 & $2 \mathrm{M}$ & $\mathrm{W}$ & 9 & $\ldots$ & 0.2 & 0.08 & 0.06 & 0.09 & Norm & 10.00 & 0.93 \\
\hline 55 & 2MASX J14482512+3559462 & 0.1133 & $2 \mathrm{M}$ & $\mathrm{W}$ & 16 & $\ldots$ & 3.9 & 0.08 & $? ?$ & $? ?$ & HDD & 0.50 & 0.60 \\
\hline 56 & [GH2004] 9 & 0.1952 & $2 \mathrm{M}$ & $\mathrm{W}$ & $\ldots$ & $\ldots$ & 0.0 & 0.00 & $? ?$ & $? ?$ & Norm & 2.50 & 0.75 \\
\hline 57 & Mrk 417 & 0.0328 & $2 \mathrm{M}$ & $\mathrm{W}$ & 2,3 & $\ldots$ & 6.7 & 0.01 & 0.36 & 0.30 & Norm & 1.00 & 0.51 \\
\hline 58 & Mrk 668 & 0.0770 & $2 \mathrm{M}$ & $\mathrm{W}$ & 1 & $\ldots$ & 2.8 & 0.08 & 0.78 & 0.95 & Norm & 0.50 & 0.33 \\
\hline 59 & NGC $4235^{\mathrm{b}}$ & 0.0075 & $2 \mathrm{M}$ & $\mathrm{W}$ & 2,14 & $\ldots$ & 7.1 & 0.10 & 0.23 & 0.26 & Norm? & $3.25 ?$ & $0.80 ?$ \\
\hline 60 & 2MASX J12384342+0927362 & 0.0829 & $2 \mathrm{M}$ & $\mathrm{W}$ & $\ldots$ & $\ldots$ & 1.4 & 0.01 & 0.14 & 0.15 & HDD & 3.00 & 0.92 \\
\hline 61 & 2MASS J10405880+5817034 & 0.0712 & $2 \mathrm{M}$ & $\mathrm{W}$ & 18 & $\ldots$ & 7.0 & 0.07 & 0.16 & 0.15 & Norm & 1.25 & 0.58 \\
\hline 62 & SDSS J164019.66+403744.4 & 0.1512 & $2 \mathrm{M}$ & $\mathrm{W}$ & 18 & $\ldots$ & 3.3 & 0.10 & 0.38 & 0.49 & Norm & 1.50 & 0.62 \\
\hline 63 & Mrk 50 & 0.0239 & $2 \mathrm{M}$ & $\mathrm{W}$ & 2,3 & $\ldots$ & 3.2 & 0.04 & $? ?$ & $? ?$ & HDD & 0.00 & 0.00 \\
\hline 64 & 2dFGRS TGN404Z026 & 0.0329 & $2 \mathrm{M}$ & W & 2,3 & $\ldots$ & 5.1 & 0.00 & 0.44 & 0.35 & Norm & 0.50 & 0.33 \\
\hline 65 & Mrk 771 & 0.0636 & $2 \mathrm{M}$ & W & 12 & $\ldots$ & 5.5 & 0.00 & 0.45 & 0.34 & Norm & 0.75 & 0.44 \\
\hline
\end{tabular}

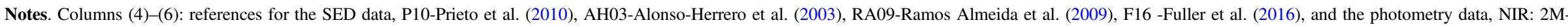

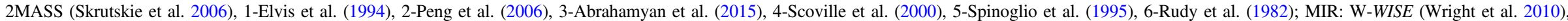

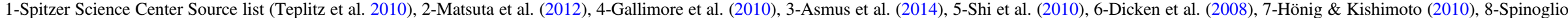

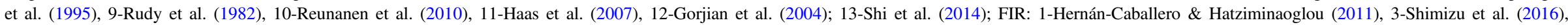

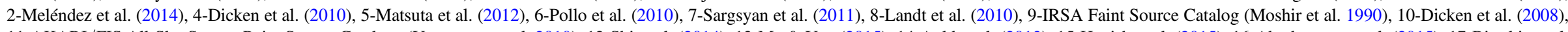

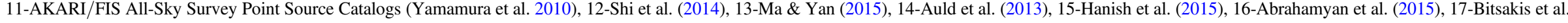

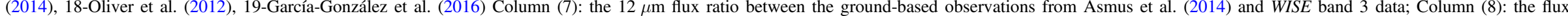

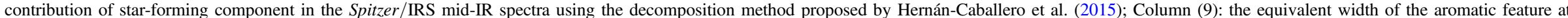

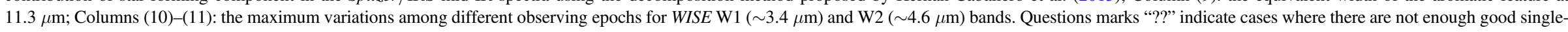

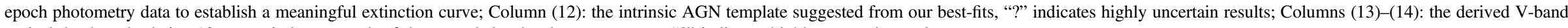
optical depth and relative $10 \mu \mathrm{m}$ emission strength of the extended polar dust component, "?" indicates highly uncertain results.

${ }^{a}$ These values are based on the hot-dust-obscured (HDO) AGN model introduced in Section 5.3.

${ }^{b}$ The duplication of NGC 4235 serves as an example to demonstrate the uncertainties caused by strong host galaxy contamination on the SED fitting results. 


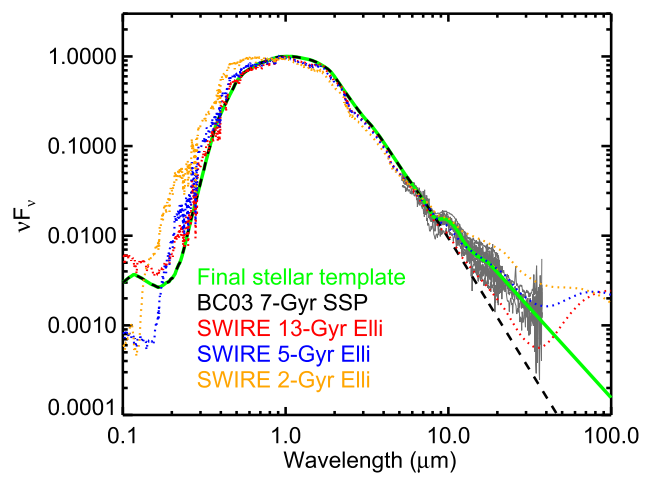

Figure 4. Comparisons of different stellar templates. We derive an empirical template by replacing a Bruzual \& Charlot (2003) 7 Gyr SSP SED template with the mid-IR $(6-20 \mu \mathrm{m})$ stellar template and a power-law $\operatorname{SED}(\lambda>20 \mu \mathrm{m})$. We plot the individual Spitzer/IRS spectra used to derived the mid-IR template as gray lines. The original Bruzual \& Charlot (2003) and the elliptical galaxy templates with age 2, 5, and 13 Gyr from the SWIRE library (Polletta et al. 2007) are also presented.

Table 3 summarizes the information for the Seyfert-1 nuclei studied in this work.

\subsection{SED Fitting Method}

\subsubsection{Stellar Emission Template}

While the galaxy star formation contribution to the mid-IR has been minimized by our sample selections, stellar contamination in the near-IR can be significant, especially for the SDSS/Spitzer AGNs. Luckily, the near-IR bands of low- $z$ galaxies are dominated by old stellar populations that share nearly identical broad-band SEDs. Despite the likely multiple stellar populations with different star formation histories, the stellar photospheric SEDs of these galaxies peak at $\sim 1 \mu \mathrm{m}$ and drop quickly following a Rayleigh-Jeans tail toward the midIR (e.g., Polletta et al. 2007). It is known that dust around evolved stars could have additional emission that supplements the quickly dropping photospheric SED at $\lambda \gtrsim 7 \mu \mathrm{m}$, particularly in early-type galaxies (e.g., Bressan et al. 2006; Rampazzo et al. 2013). For some low-luminosity AGNs, where the stellar contamination can even contribute to the mid-IR, we need more accurate templates.

There are several stellar population synthesis models that include the effect of circumstellar dust shells from evolved stars (e.g., Bressan et al. 1998; Silva et al. 1998; Piovan et al. 2003; González-Lópezlira et al. 2010; Cassarà et al. 2013; Villaume et al. 2015). However, due to the lack of constraints on the metallicity, mass loss rates or properties of the dust shells around the evolved stars in the AGN host galaxies, we decided to derive an empirical template. Following Hernán-Caballero et al. (2015), we used the Spitzer/IRS spectra of 18 local earlytype galaxies with negligible indication of star formation activities $^{3}$ to derive a mean mid-IR stellar template at 6-20 $\mu \mathrm{m}$ with the normalization at $7 \mu \mathrm{m}$. Only a few of them have LL2 (19.9-39.9 $\mu \mathrm{m})$ observations but the spectral slope can be roughly described by a power law $f_{\nu} \propto \nu^{1.0}$ at $\lambda>20 \mu \mathrm{m}$. We continue this mid-IR stellar template as a power law at longer wavelengths and join it to the $7 \mathrm{Gyr}$ single stellar population

\footnotetext{
3 This early-type galaxy sample includes: NGC 4474, NGC 4377, NGC 4564, NGC 4570, NGC 4660, M 85, NGC 4473, NGC 5812, NGC 1700, NGC 1374, NGC 0821, NGC 5831, NGC 1297, NGC 1366, NGC 3818, NGC 7332, NGC 1549, and NGC 3904.
}

(SSP) template from Bruzual \& Charlot (2003) at $7.0 \mu \mathrm{m}$. The results can be seen in Figure 4, together with three elliptical galaxy templates generated with the GRASIL code (Silva et al. 1998) by Polletta et al. (2007). Compared with the original dust-free Bruzual \& Charlot (2003) template, the starheated dust features increase the flux by a factor of $\gtrsim 1.5-3$ at $\lambda>10 \mu \mathrm{m}$. At $\lambda \sim 1-8 \mu \mathrm{m}$, the SEDs of different templates are remarkably similar, so the choice of the Bruzual \& Charlot (2003) templates has little effect on the IR SED modeling.

\subsubsection{AGN Templates}

For the AGN component, a series of semi-empirical SEDs is produced with the model introduced in Section 2. Because our major goal is to test if Seyfert nuclei behave similarly to NGC 3783, the radial density profile and dust grain properties of the extended dust component are fixed to the values in Table 2. All three AGN intrinsic templates presented in Lyu et al. (2017) are obscured with an increment of 0.25 in optical depth $\left(\tau_{\mathrm{V}}\right)$. We end up with 123 AGN templates that cover $\tau_{\mathrm{V}}=0-10$.

\subsubsection{Other Far-infrared Components}

More than $80 \%$ of our sample have constraints on the far-IR SEDs. We used Rieke et al. (2009) star-forming galaxy (SFG) templates to describe the host galaxy dust emission. This library was developed based on Spitzer observations of local galaxies and each template is parameterized by a different IR luminosity $\log \left(L_{\mathrm{SF}, \text { temp }} / L_{\odot}\right)$. We decide to adopt a default value of $\log \left(L_{\mathrm{SF}, \text { temp }} / L_{\odot}\right)=10.75$ for most objects.

Some radio-loud AGNs can have strong synchrotron radiation in the far-IR bands. We assume a broken power-law model (see, e.g., Pe'er 2014) to represent this contribution

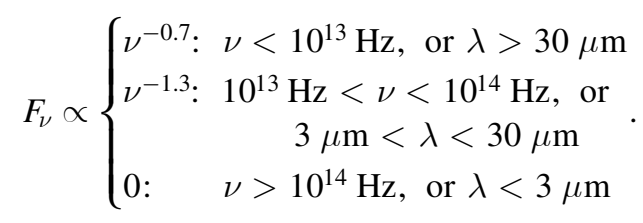

\subsubsection{SED Models}

Our model would predict obscuration in the UV-optical band for many AGNs. However, this effect will be dependent on our LOS; any clumpiness in the distribution of the polar dust will result in variations in the extinction. Indeed, it is possible to imagine a system where the polar dust covers $99 \%$ of the sky as seen by the central engine and hence provides an important infrared SED component, but where our LOS by chance falls along the $1 \%$ of directions without dust and hence the central engine appears to be unobscured. In light of this, we only focus on the IR SEDs for individual objects. To reduce the influence of host galaxy star formation contamination, the fittings are limited to $\lambda \sim 1-30 \mu \mathrm{m}$.

We combined the (reddened) AGN templates with the stellar templates and used $\chi^{2}$ minimization to fit the SEDs. Since the data have a range of nonstatistical errors, a uniform weight for all bands is assumed instead of using the quoted flux uncertainties. There are only four free parameters in our model: the normalizations of the stellar and the AGN templates, the type of the AGN template, and its optical extinction $\tau_{\mathrm{V}}$. The best AGN template is selected by searching the combination with the minimum $\chi^{2}$ value among all three sets of reddened 

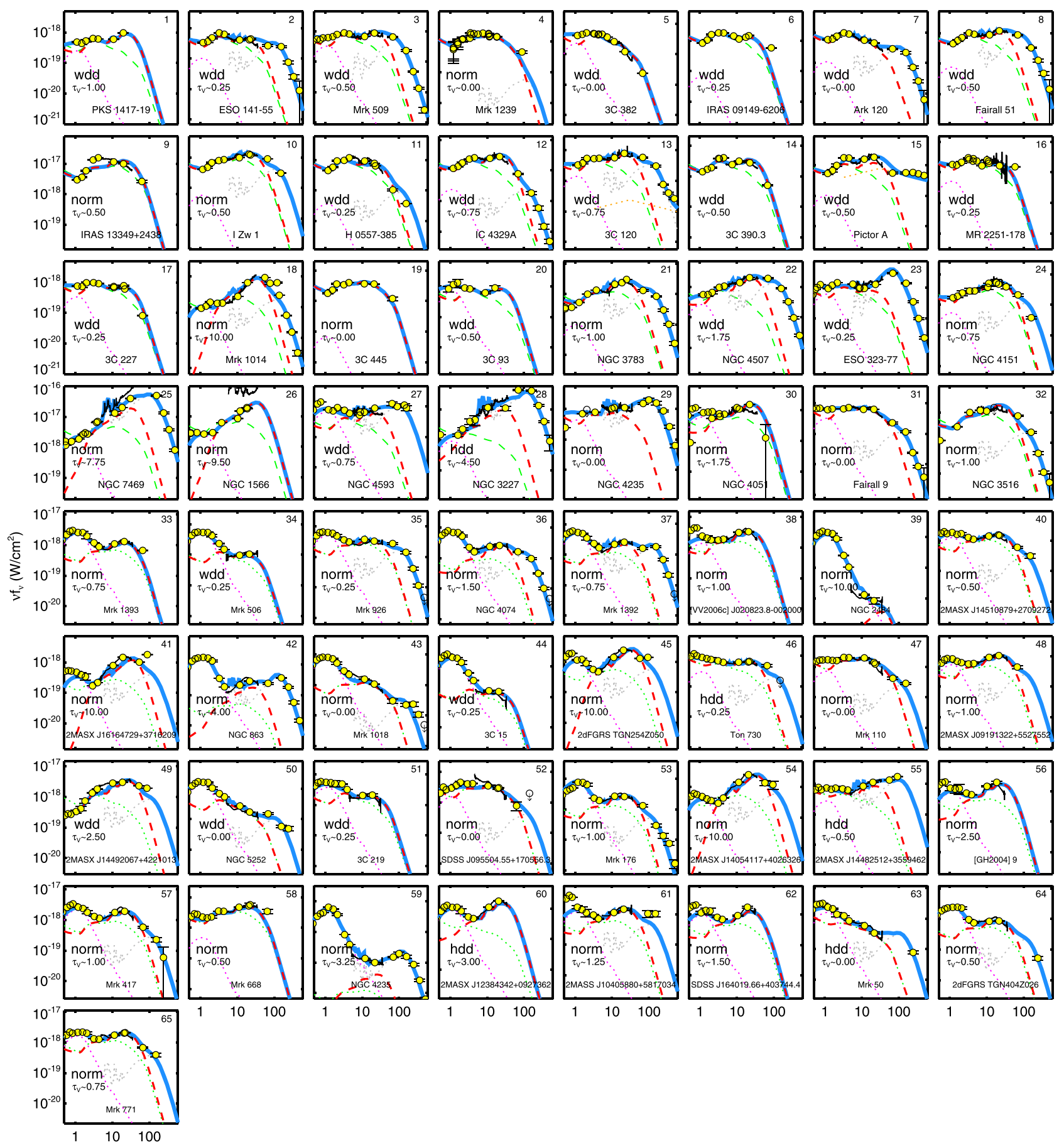

$\lambda(\mu \mathrm{m})$

Figure 5. Best-fit results for low-z Seyfert-1 nuclei with the reddened AGN model trained for NGC 3783. Photometric data points are shown as yellow dots and the Spitzer/ IRS mid-IR spectra are plotted as black solid lines. The SED model (blue thick solid lines) is composed of the AGN component (red dashed lines), the stellar component (magenta dotted lines), and the far-IR star formation component (gray dotted lines). For 3C 120 and Pictor A, the synchrotron emission component (orange dotted lines) is added into the fittings. We also plot the suggested intrinsic AGN template (green dashed lines) for each object from our SED fittings to compare the observed SED.

AGN libraries (normal, WDD, and HDD). However, if $\chi^{2}$ with the best reddened normal AGN template is less than 1.5 times that with the best reddened dust-deficient template, the normal AGN template is selected.

For objects with at least two photometry data points at $\lambda>50 \mu \mathrm{m}$, a second-round SED fitting at $\lambda \sim 1-500 \mu \mathrm{m}$ was carried out by combining the AGN template selected above, a stellar template and an SFG far-IR template. For eight objects, we changed the default SFG template to another (Rieke et al.
2009) template with a lower or higher $\log \left(L_{\mathrm{SF} \text {,temp }} / L_{\odot}\right)$ in the range of $9.75-10.50$ to improve the fitting of the far-IR SED peak. For two objects, Pictor A and 3C 120, the synchrotron emission template was added to reproduce their far-IR and submm SEDs.

\subsection{Fitting the IR SEDs of Individual Objects}

The best-fit results for type-1 nuclei can be seen in Figure 5 . The SED shape of the AGN component is only determined by 


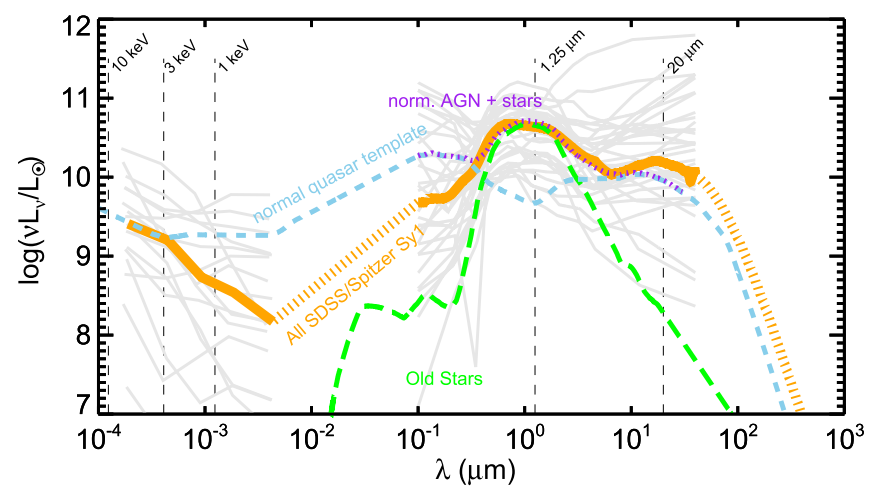

Figure 6. The individual SEDs (gray thin lines) and the median composite SEDs (orange thick line) of the SDSS-Spitzer/IRS Seyfert-1 AGN sample. As a comparison, we also plot the intrinsic AGN template for normal quasars (dashed blue line) from $\mathrm{Xu}$ et al. (2015). We used the modified Bruzual \& Charlot (2003) stellar template with age $~ 7$ Gyr (green long-dashed line) and the normal AGN template (blue dashed line) to reproduce the composite SED at $0.5-3.0 \mu \mathrm{m}$ with the model as a magenta dotted line.

two free parameters: the type of the intrinsic template (normal, WDD, or HDD) and the optical depth, $\tau_{\mathrm{V}}$, of the polar dust component. Surprisingly, this simple model fits the broad-band IR SEDs of most objects reasonably well. The only notable exceptions are IRAS $13349+2438$ and 2MASX J14492067 +4221013, whose SEDs feature strong hot dust excess emission. As shown later (Section 5), similar characteristics can be found in the so-called hot DOGs, whose SEDs our model can fit by adjusting the density profile of the polar dust.

We want to mention several complications that could influence the quality of the SED fitting. First, AGNs show near- and mid-IR variability that could produce a gap between different datasets. In fact, $\gtrsim 40 \%$ of the Seyfert sample have strong mid-IR variability with $\Delta W 1>0.3$ mag during the available WISE epochs, in contrast with $\sim 13 \%$ for the Palomar-Green (PG) quasar sample in our previous study (Lyu et al. 2017). second, it is quite likely that the dust has different compositions or distributions among different objects. Indeed, we can improve the fittings by sampling more parameter space with similar MCMC methods used for NGC 3783 (see Section 2.4). However, the exact situations for these properties cannot be observationally constrained. Our goal is to reproduce a wide range of observations in a way that minimizes the uncertainties due to model degeneracy, not to produce another complicated model that may fit a few observations perfectly.

In summary, our four-free-parameter simple model provides reasonably good fits in the near-IR and mid-IR to nearly the entire sample of AGNs.

\subsection{Testing the Reddened AGN Templates with Composite SEDs}

We now work with SEDs averaged over the SDSS-Spitzer/ IRS sample to test the prediction that objects with excess IR emission due to polar dust should have obscuration in some directions. By averaging over a sample of AGNs, evidence of such obscuration should be obvious even if it is very nonuniform for individual sources.

\subsubsection{Composite SEDs of the SDSS Seyfert-1 Nuclei}

For each object, we corrected the flux for the foreground Galactic extinction in the UV-optical bands according to the SFD dust map (Schlegel et al. 1998; Schlafly \& Finkbeiner 2011). The observed flux is converted into luminosity and the SED is smoothed with a $\Delta \log (\nu)=0.2$ boxcar in the $\lambda-\lambda L_{\lambda}$ space. The median of all these SEDs is then computed to produce a composite SED. To reduce the uncertainties in the mid-IR due to the low resolution of photometry data points, we also derive a median mid-IR spectrum of the sample using the Spitzer/IRS data in a similar fashion. In the last step, we replace the 6-35 $\mu \mathrm{m}$ low-resolution SED template based on photometric data with the median mid-IR spectrum of the sample.

Figure 6 presents the median SED for the whole SDSSSpitzer/IRS Seyfert-1 sample. Since we do not remove the near-IR host galaxy contamination, a near-IR SED bump due to emission from an old stellar population is expected. In the midIR, this Seyfert-1 median SED presents stronger emission at $\lambda \gtrsim 10 \mu \mathrm{m}$ compared with the near-IR normalized normal Elvis-like template. Since any objects with strong host galaxy contamination in the mid-IR have been dropped, such excess emission has to be AGN-dominated. On the other hand, the UV and soft X-ray (to $\sim 3 \mathrm{keV}$ ) composite SED of this Seyfert-1 sample is suppressed relative to the quasar template. Beyond $3 \mathrm{keV}$, the two templates are similar. This UV-optical dip is the expected signature of obscuration in these Seyfert-1 nuclei. It is an indication that the Seyfert-1 mid-IR excess is from the reradiation of the absorbed energy by the dust that also provides the short-wavelength obscuration.

With the SDSS/Spitzer sample, we also demonstrate that both normal AGNs and dust-deficient AGNs exist in the Seyfert-1 population, which supports the idea that similar intrinsic AGN templates are also valid for relatively lowluminosity AGNs. Based on the mid-IR continuum shape, objects with similar IR SED characteristics are grouped together and a composite SED is derived similarly to the whole sample median SED. We classify these AGNs into three categories and show that their composite SEDs have the following characteristics compared with the Elvis et al. (1994)like templates for normal AGNs:

1. $(\mathrm{C} 1, N=5)$ normal AGNs. These objects have SEDs well reproduced by the normal AGN template with some stellar contribution in the near-IR. Some of them have a little far-IR excess emission, which might be associated with the host galaxy.

2. $(\mathrm{C} 2, N=7)$ dust-deficient AGNs. These objects present a deficiency of the dust emission at $\lambda \gtrsim 5 \mu \mathrm{m}$. However, since the stellar contribution in the near-IR cannot be calibrated, it is hard to argue this SED is HDD or WDD. It is likely a combination of both populations.

3. (C3, $N=20)$ warm-excess AGNs. These objects feature a mid-IR bump peaking around $\lambda \sim 20 \mu \mathrm{m}$, in contrast with the flat $\sim 3-20 \mu \mathrm{m}$ continuum of normal quasars. A similar mid-IR feature is also present in the SED of NGC 3783 as well as the composite SEDs of the whole sample.

These results are presented in Figure 7: (1) In the top panels we demonstrate using the Spitzer/IRS spectra to construct composite spectra in the $6-30 \mu \mathrm{m}$ range. (2) In the middle panels we merge the mid-IR spectral templates with composite 

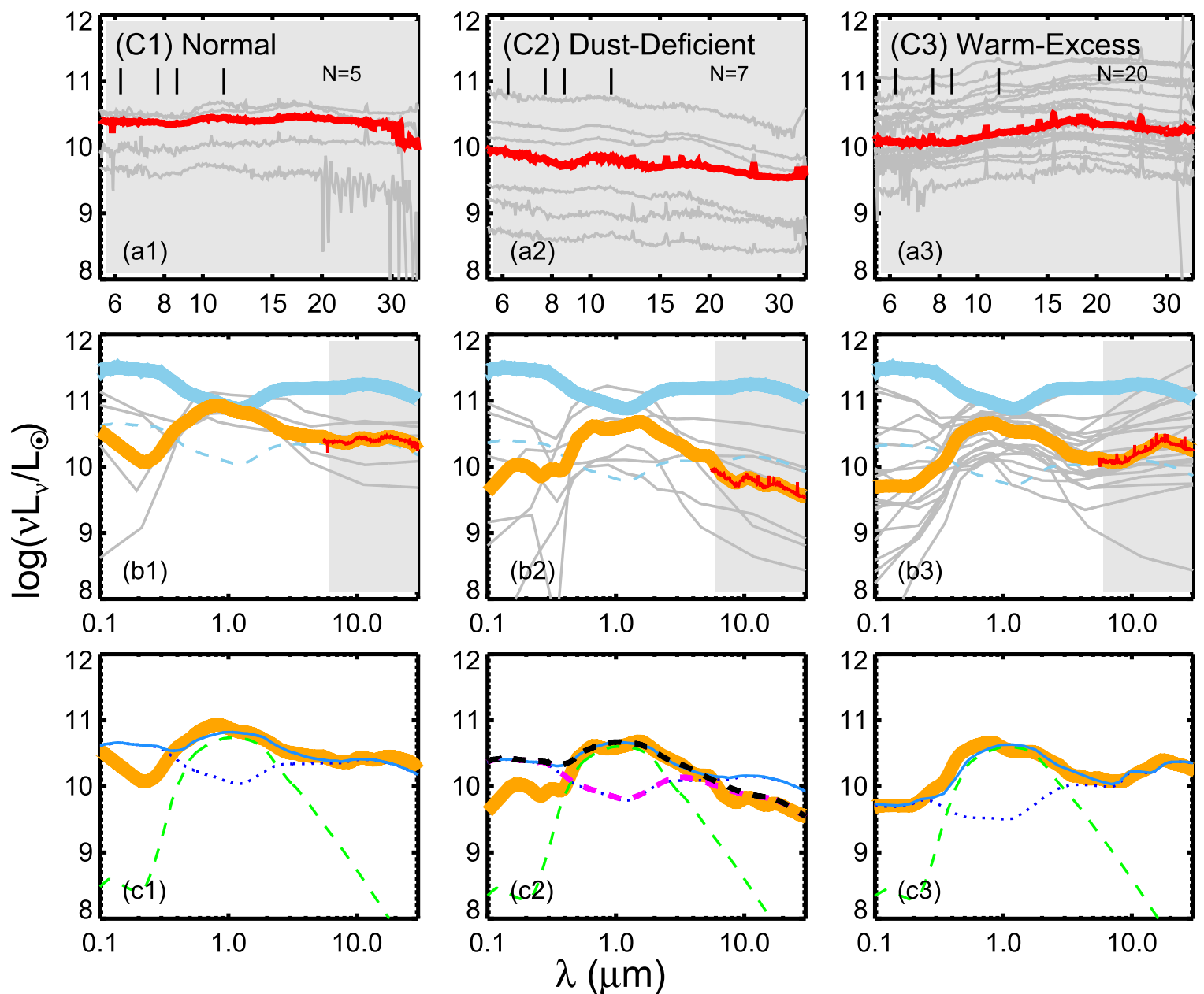

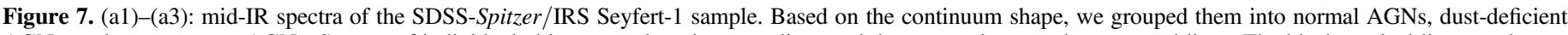

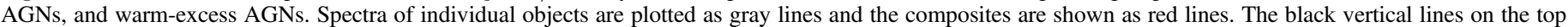

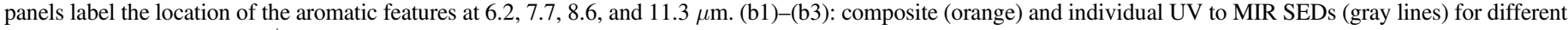

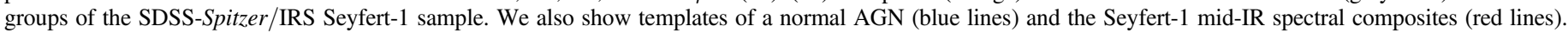

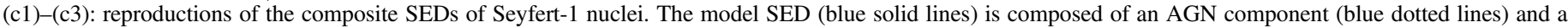

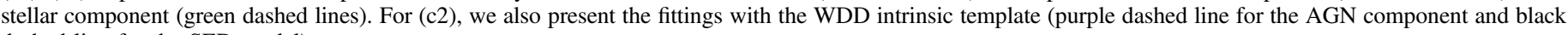
dashed line for the SED model).

SEDs at the shorter wavelengths built primarily using photometry. The middle right panel shows the mid-IR bump clearly when compared with the left and center panels. (3) The bottom panels present our model fits (see below).

\subsubsection{Reproducing the Composite SEDs}

For the composite templates (C1) and (C2), due to the relatively small sample sizes $(N<10)$, the UV-optical SEDs may not be representative of the overall extinction and suffer the possibility of variability. However, either the normal AGN template or the dust-deficient AGN template plus the stellar template can reproduce the Seyfert IR SED.

For (C3), we apply the reddened AGN templates to fit the average composite SEDs together with the stellar template. This UV to mid-IR composite SED is matched by the reddened WDD template with $\tau_{\mathrm{V}}=1.5$ and all other parameters of the polar dust component fixed the same for NGC 3783.

We conclude that the unobscured quasar templates can be directly applied to some Seyfert-1 nuclei ( $\sim 37 \%$ in our case). Both normal AGNs and dust-deficient AGNs are seen among the Seyfert-1 population. Meanwhile, most Seyfert-1 nuclei present evidence for low- to moderate-level obscuration from the soft X-ray to the optical with some dust excess emission starting from the mid-IR, which can be reproduced by our polar-dust-obscured AGN template. These results support our model assumptions introduced in Section 2.

\section{Characterizing the AGN Polar Dust Emission}

In Section 2.4, the SED features and the polar dust emission strength of NGC 3783 were accurately reproduced by the reddened AGN model proposed in this study. With the same parameters used for this single object, our reddened AGN templates, combined with the host galaxy templates, successfully fit the IR SEDs of another 64 Seyfert-1 nuclei and the UV to mid-IR composite SEDs of the SDSS/Spitzer type-1 AGNs (Section 3.3). In Section 3.4, we found that on average type-1 AGN SEDs show indications of extinction as predicted by our approach. These results support our proposal that the SED differences between quasars and Seyfert- 1 nuclei are due to the IR-reprocessed emission from the extended distribution of dust 

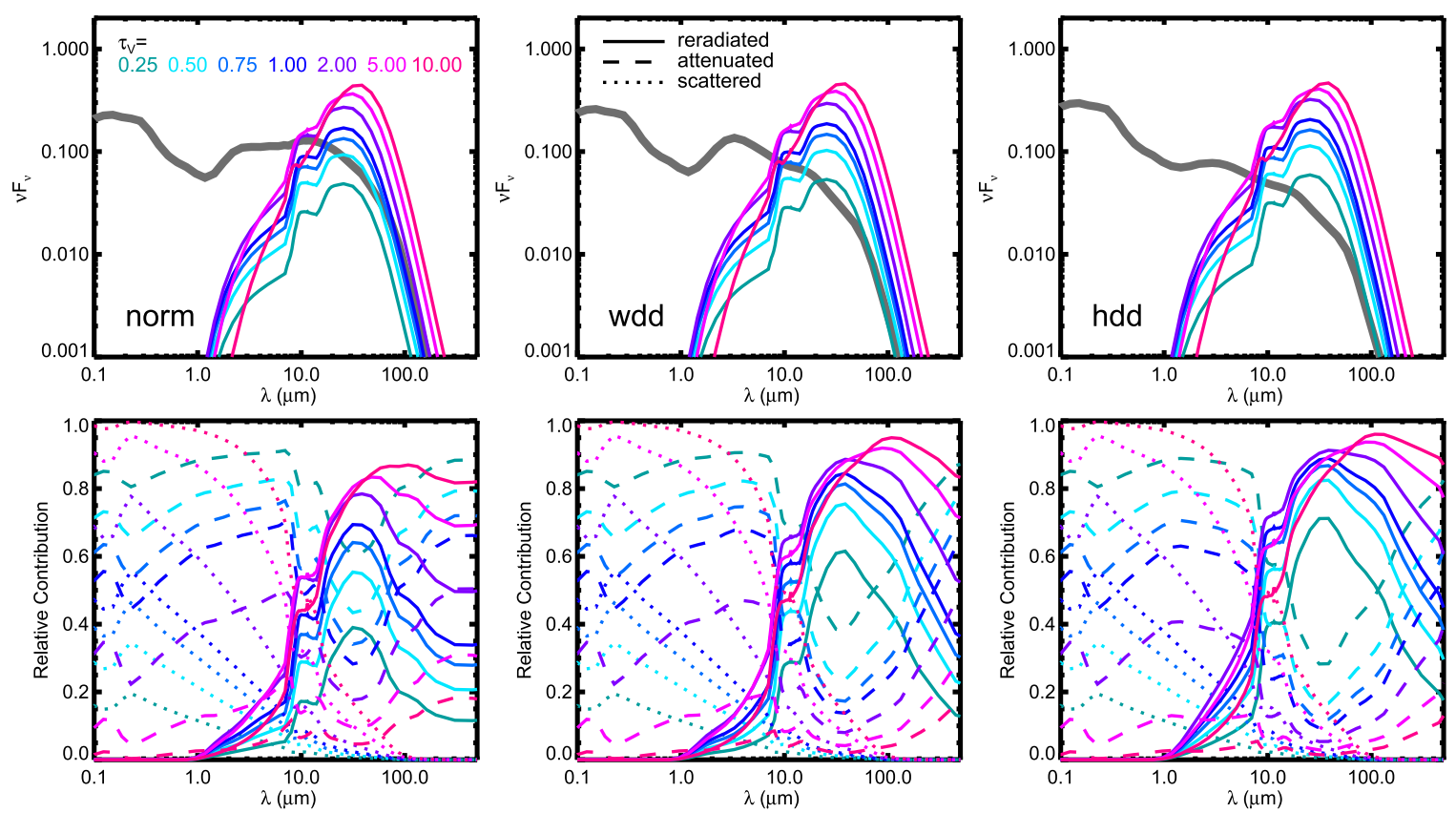

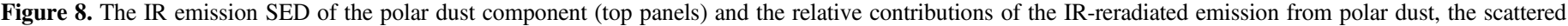

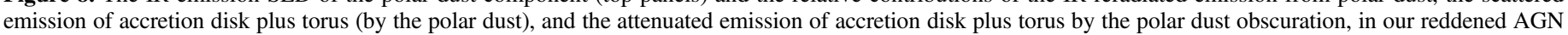
templates (bottom panels).

that is also responsible for the common low-level obscuration of the Seyfert nuclei.

Now we characterize the possible SED features of the polar dust emission and explore its prevalence among the Seyfert-1 nuclei studied in this work.

\subsection{SED Features}

In the top panels of Figure 8, we show the SEDs of the IR-reprocessed emission from the extended polar dust component as a function of $\tau_{\mathrm{V}}$ for normal, WDD, and HDD AGN. In the bottom panels, the relative contributions of the dust IR-reprocessed emission, dust-scattered emission, and the attenuated emission of the intrinsic AGN templates are compared.

There are several interesting results. First, at a given $\tau_{\mathbf{V}}$, regardless of the intrinsic AGN template, the strength and the SED shape of the polar dust emission are almost identical. This is easily understood since the configurations of the dust obscuration properties and the UV to optical SED inputs, where the emission mostly heats the dust, are the same. This result also underlies the possibility to fit a broad variety of observed SEDs with $\tau_{\mathrm{V}}$ as the only free parameter to characterize the contribution of the polar dust component.

In addition, at relatively low optical depth $\left(\tau_{\mathrm{V}}<5\right)$, although the overall strength of the polar dust emission changes with $\tau_{\mathrm{V}}$, its SED shape is identical at the wavelengths where this component matters. As discussed in Section 2.2.2, different dust-covering factors would only change the relative scaling of the dust emission SED. Thus, we can always change the value of $\tau_{\mathrm{V}}$ to match the effect caused by the dust-covering factor on the final SED shape, as long as $\tau_{\mathrm{V}}$ is not so large that the polar dust emission becomes optically thick in the mid-IR.

Thus, we can describe the AGN-heated polar dust emission with one single template when the integrated optical depth is modest $\left(\tau_{\mathrm{V}} \lesssim 5\right)$. In fact, $\sim 90 \%$ of our low- $z$ Seyfert-1 AGN sample is fitted by the reddened AGN templates with $\tau_{\mathrm{V}} \leqslant 5$.

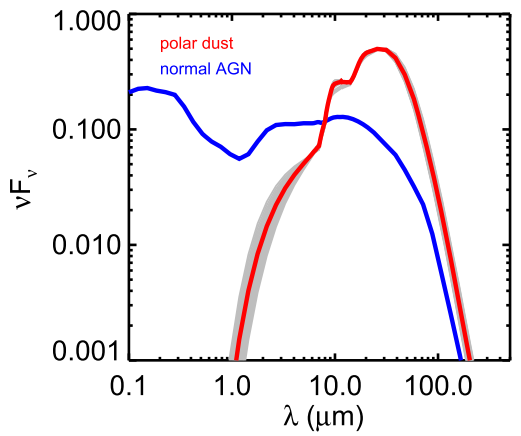

Figure 9. IR SED template for AGN polar dust emission (red). We also plot the intrinsic normal AGN template as a comparison (blue). The gray region represents the range of SED variations of polar dust emission with the normalizations at $26.5 \mu \mathrm{m}$ and $\tau_{\mathrm{V}} \sim 0-5$.

We suggest that this polar dust template can be generally used to further reduce the number of free parameters in the SED fittings.

Figure 9 shows the SED template for the polar dust emission. Its emission is peaked at $\sim 25.6 \mu \mathrm{m}$, corresponding to a characteristic dust temperature of $\sim 113 \mathrm{~K}$. In other words, most of the energy from the central engine absorbed by the polar dust will be radiatively transferred into the mid- to far-IR bands. As we discussed in Section 2, the polar dust should consist of large grains, e.g., $a_{\min } \sim 0.04 \mu \mathrm{m}$ and $a_{\max } \sim 10 \mu \mathrm{m}$. We use the SKIRT code (Baes et al. 2003, 2011) to compute the optical properties of the polar dust by calculating the total extinction cross section averaged over such a standard grain size distribution with 20 bins for silicate and graphite with a mixture $0.53: 0.47$. Then an extinction is derived by normalizing the polar dust optical properties at the $\mathrm{V}$ band $(0.55 \mu \mathrm{m})$ and shown in Figure 10 . Due to the presence of $a \gtrsim 0.3 \mu \mathrm{m}$ grains, the mid-IR extinction, e.g., $A_{10 \mu \mathrm{m}}$, is at least two orders of magnitude lower relative to the optical extinction, $A_{\mathrm{V}}$. Since the majority of our 


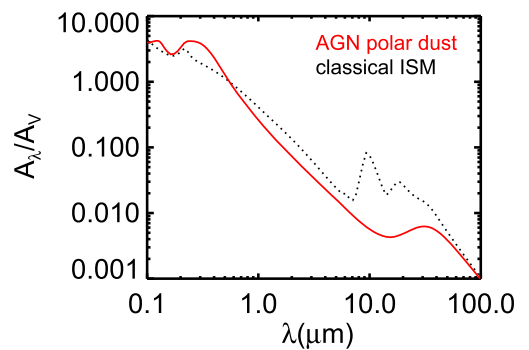

Figure 10. Extinction curves of suggested AGN polar dust (red solid line) and classical ISM dust (black dashed line).

sample yields $A_{\mathrm{V}} \lesssim 5$, the possible extinction at the wavelengths where the polar dust emits the most energy is extremely low. In this case, the shape of its emission SED would not be influenced by the geometry of the polar dust component but only depend on its radial density profile, as explained in Section 2.2.2.

\subsection{Observed Size}

Figure 11 presents the radial profiles of the temperature distribution for the extended polar dust component in our model. Similar to the IR-reprocessed emission SEDs, the temperature profiles also have little dependence on the optical depth $\left(\tau_{\mathrm{V}}\right)$. With the Wien displacement law, we relate some typical observed wavelengths to the dust temperature and estimate the corresponding physical size. In the near-IR $(\sim 2.2 \mu \mathrm{m})$, we can only see the very inner part $\left(r_{\text {out }, \text { obs }} \sim 1.3 r_{\text {in }}\right)$ of the extended dust component. In the midIR $(\sim 8-13 \mu \mathrm{m})$, the observed size increases to $r_{\text {out obs }} \sim$ $20-41 r_{\text {in }}$. At $\lambda \sim 26 \mu \mathrm{m}$, the polar dust emission reaches its peak, corresponding to an observed size of $r_{\text {out,obs }} \sim$ 200-300 $r_{\text {in. }}$ At even longer wavelengths, the observed size of dust distribution is expected to change slowly. However, since its SED drops quickly as a power law, the polar dust emission would be very faint and spreads out in a relatively extended area, making any detections challenging.

Considering the AGN polar dust distribution could have some dust-covering factor and its morphology will depend on the observing angle, the estimations provided here should be considered only as order-of-magnitude values.

\subsection{The Prevalence of AGNs with Strong Polar Dust Emission}

As presented in the bottom panels of Figure 8, the relative strength of the reprocessed and scattered emission by the polar dust is a function of the optical depth $\tau_{\mathrm{V}}$. We take the relative contribution of the these two components at $10 \mu \mathrm{m}$, $f_{\text {pol, } 10 \mu \mathrm{m}} \gtrsim 0.5$ as the threshold for AGNs with significant polar dust emission. However, the exact values cannot be definitive since there are several sources of uncertainties. First, the intrinsic IR emission of the AGNs presents variations and there could exist objects with intermediate SEDs that cannot be unambiguously grouped into normal, WDD, or HDD AGNs (Lyu et al. 2017). In such cases, the SED model would not make a decisive selection of the intrinsic AGN type, making the value of $f_{\mathrm{pol}, 10 \mu \mathrm{m}}$ uncertain. In addition, despite the model simplicity, fitting degeneracy still exists. A good example is NGC 3783, where our SED fittings in Section 3.3 suggest the intrinsic AGN emission can be also represented by a normal AGN template with $f_{\mathrm{pol}, 10 \mu \mathrm{m}} \sim 0.50$, which is much

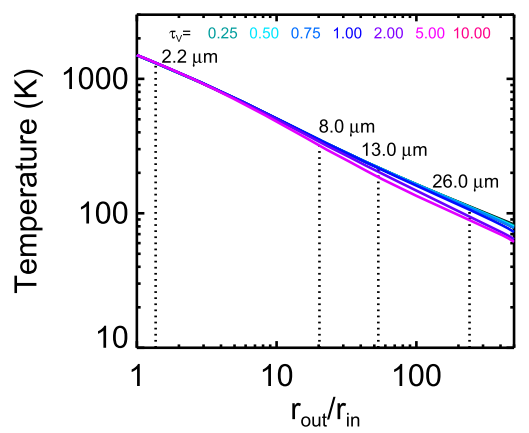

Figure 11. Radial profiles of the dust temperature of the extended dust component with $\tau_{\mathrm{V}}=0.25,0.50,0.75,1.00,2,5$, and 9.75 . The geometry structure and grain properties are the same as for NGC 3783 (see Table 2). We denote the radii that dominate the emission at four typical wavelengths by the vertical dotted lines.

lower than the Hönig et al. (2013) mid-IR interferometry measurements. Last but not the least, as shown by the example of NGC 4235 (see the fittings \#29 and \#59 in Figure 5), if the SED were strongly contaminated by the galaxy emission, the derived AGN component could be highly uncertain, making the identification of polar dust emission specious.

To get a conservative number of significant polar dust emitters, we remove objects with significant near-IR stellar contamination and count the fittings with the reddened normal AGN templates only. In this way, the fraction of objects with significant polar dust emission among the HSR Seyfert-1 sample is $13 \%-31 \%$. For the SDSS/Spitzer sample, this value is $36 \%-45 \%$. In Table 4, we list the candidate objects with significant polar dust emission. This behavior appears to be characteristic for at least $25 \%-38 \%$ of relatively lowluminosity type-1 AGNs in Seyfert galaxies.

Based on the SED analysis, we can also put an upper limit on the fraction of objects with polar dust emission. As argued in Section 2.1, an AGN whose SED can be directly matched by any of the Lyu et al. (2017) intrinsic templates (i.e., $\tau_{\mathrm{V} \text {,ext }} \sim 0$ ) is unlikely to have strong polar dust emission. There are 11 such objects in our whole sample, making the polar-dust-free sample fraction about $\sim 20 \%$. There are another 11 objects with $\tau_{\mathrm{V}, \text { ext }} \sim 0.25$, or $f_{\text {pol, } 10 \mu \mathrm{m}} \lesssim 30 \%$. Accordingly, the proportion in our Seyfert-1 sample with significant polar dust emission should be no more than $\sim 70 \%$, assuming that the values of $f_{\text {pol, } 10 \mu \mathrm{m}}$ were underestimated among half of the samples with moderate polar dust emission.

Lastly, note that our intrinsic AGN templates are the descriptions of common features and the IR SEDs of polardust-free AGNs are expected to have additional variations among individual objects (e.g., see Figure 10 in Lyu et al. 2017). Bearing this in mind, we may conclude that about $1 / 3$ of the type- 1 nuclei studied in this work show evidence for strong emission by polar dust, about $1 / 3$ show no evidence, and the remaining $1 / 3$ has weaker or absent emission-they are ambiguous.

\subsection{Comparison with Mid-IR Morphology-based Polar Dust Identification}

Among the type-1 AGNs studied in this work, there are 13 objects whose mid-IR morphology has been studied via interferometry by López-Gonzaga et al. (2016). Ten of them, Mrk 1239, IRAS 09149-6206, IRAS 13349+2438, I Zw 1, H 
Table 4

Candidate AGNs with Significant Polar Dust Emission

\begin{tabular}{|c|c|c|c|c|}
\hline ID & Name & Type & $f_{\mathrm{pol}, 10 \mu \mathrm{m}}$ & Morph. \\
\hline \multicolumn{5}{|c|}{ HSR AGN Sample } \\
\hline 1 & PKS 1417-19 & WDD & 0.65 & $\mathrm{p}$ \\
\hline 12 & IC 4329A & WDD & 0.58 & $\mathrm{p}, \mathrm{U}$ \\
\hline 13 & $3 \mathrm{C} 120$ & WDD & 0.58 & $\mathrm{u}$ \\
\hline 18 & Mrk 1014 & Norm & 0.93 & $\mathrm{p}$ \\
\hline 21 & NGC 3783 & Norm & 0.51 & $\mathrm{u}, \mathrm{E}$ \\
\hline 22 & NGC 4507 & WDD & 0.78 & $\mathrm{n}, \mathrm{C}$ \\
\hline 25 & NGC 7469 & Norm & 0.91 & $\mathrm{y}, \mathrm{U}$ \\
\hline 26 & NGC 1566 & Norm & 0.93 & $\mathrm{u}$ \\
\hline 27 & NGC 4593 & WDD & 0.58 & $\mathrm{y}, \mathrm{U}$ \\
\hline 28 & NGC 3227 & HDD & 0.94 & $\mathrm{y}$ \\
\hline \multicolumn{5}{|c|}{ SDSS/Spitzer AGN Sample } \\
\hline 36 & NGC 4074 & Norm & 0.62 & $\mathrm{p}$ \\
\hline 38 & [VV2006c] J020823.8-002000 & Norm & 0.51 & $\ldots$ \\
\hline 41 & 2MASX J16164729+3716209 & Norm & 0.93 & $\ldots$ \\
\hline 42 & NGC 863 (aka Mrk 590) & Norm & 0.84 & $\mathrm{n}$ \\
\hline 45 & 2dFGRS TGN254Z050 & Norm & 0.93 & $\cdots$ \\
\hline 48 & 2MASX J09191322+5527552 & Norm & 0.51 & $\ldots$ \\
\hline 49 & 2MASX J14492067+4221013 & WDD & 0.84 & $\ldots$ \\
\hline 53 & Mrk 176 & Norm & 0.51 & $\ldots$ \\
\hline 54 & 2MASX J14054117+4026326 & Norm & 0.93 & $\ldots$ \\
\hline 55 & 2MASX J14482512+3559462 & HDD & 0.60 & $\ldots$ \\
\hline 56 & [GH2004] 9 & Norm & 0.75 & $\ldots$ \\
\hline 57 & Mrk 417 & Norm & 0.51 & $\cdots$ \\
\hline 60 & 2MASX J12384342+0927362 & HDD & 0.92 & $\ldots$ \\
\hline 61 & 2MASS J10405880+5817034 & Norm & 0.58 & $\ldots$ \\
\hline 62 & SDSS J164019.66+403744.4 & Norm & 0.63 & $\ldots$ \\
\hline
\end{tabular}

Note. These candidates are selected from our SED modeling. Morph.-nuclear mid-IR extension derived from single-disk images by Asmus et al. (2014): nnot resolved, $\mathrm{p}$ - possibly extended, $\mathrm{u}$ - unknown extension, $\mathrm{y}$ - extended, and interferometry observations by López-Gonzaga et al. (2016): C — circular, Eelongated, U-marginally resolved or unresolved $12 \mu \mathrm{m}$ emission.

0557-385, IC 4329A, ESO 323-77, NGC 4151, NGC 7469, and NGC 4593, are marginally resolved or unresolved at $\sim 12 \mu \mathrm{m}$. For one object, NGC 1566, the result is uncertain. Conclusive arguments on the existence of their polar dust emission cannot be reached due to limited UV coverage and signal-to-noise ratios. From our SED analysis, NGC 7469 and NGC 1566 may present very prominent polar dust emission with $f_{\text {pol, } 10 \mu \mathrm{m}} \gtrsim 0.9$; IC 4329A, NGC 4151, NGC 4593, and I Zw 1 have $f_{\text {pol, } 10 \mu \mathrm{m}} \sim 0.4-0.6$; IRAS 09149-6206 and ESO 323-77 could be moderate polar dust emitter with $f_{\text {pol, } 10 \mu \mathrm{m}} \sim 0.3$; Mrk 1239 has an IR SED best described by the intrinsic normal AGN template so it is unlikely to have much polar dust emission. Besides NGC 3783, López-Gonzaga et al. (2016) found another type-1 AGN, NGC 4507, presents evidence for polar dust emission but with a nearly circular morphology. The strong polar dust emission of NGC 4507 is also identified by us with $f_{\text {pol, } 10 \mu \mathrm{m}} \sim 78 \%$.

Asmus et al. (2016) studied the mid-IR emission extension for a large number of low- $z$ Seyfert nuclei at subarcsecond scales. Among their objects with extended mid-IR emission that are likely associated with polar dust emission, four type-1 nuclei have been studied with SED analysis in this work: NGC 3227, NGC 4593, NGC 7469, and ESO 323-77. The first three objects have been successfully identified by us in Table 4 with $f_{\text {pol, } 10 \mu \mathrm{m}} \gtrsim 0.6$. The SED of ESO 323-77 is best fitted by the reddened WDD AGN template with $\tau_{V} \sim 0.25$ and $f_{\mathrm{pol}, 10 \mu \mathrm{m}} \sim 0.30$, suggesting the likelihood of moderate midIR polar dust emission. In fact, very recently, Leftley et al. (2018) reported interferometric observations of ESO 323-77, arguing that $\sim 35 \%$ of its flux at $8-13 \mu \mathrm{m}$ is polar extended. This is in good agreement with our SED analysis.

These consistent results demonstrate that the infrared SED analysis could be a promising and low-budget method to look for AGNs with polar dust emission.

\subsection{Type-2 AGNs}

Since most AGNs with evidence of the extended polar dust emission are type-2 objects (e.g., Asmus et al. 2016; LópezGonzaga et al. 2016), it is ideal to carry out some similar SED analyses of such objects. However, we do not have a robust understanding of the SEDs of the circumnuclear tori for these sources. Although current radiative transfer models can produce a wide range of SED features, they have too many degeneracies to be tested robustly, particularly given the likelihood of contamination by the emission of star-forming regions and AGN-heated polar dust. Consequently, we have had to leave type-2 AGN out of our study.

\section{High- $z$ Type-1 AGNs with Peculiar SED Features}

Recent observations have identified some high- $z$ type-1 AGNs with peculiar SEDs that cannot be easily reproduced by the classical AGN template with simple UV-optical reddening. Although we lack the detailed understanding of these types of object that has been accumulated for Seyfert galaxies, we show in this section how their SEDs are consistent with being shaped by polar dust that can be fitted by our model.

\subsection{Extremely Red Quasars}

From the Baryon Oscillation Sky Survey, Ross et al. (2015) identified a population of ERQs, using SDSS and WISE photometry. These objects are very luminous with AGN bolometric luminosities $\gtrsim 10^{13} L_{\odot}$, so that any host galaxy contamination at longer wavelengths can be ignored. In addition, outflows are commonly revealed by the UV to optical emission line profiles in this type of objects (Zakamska et al. 2016; Hamann et al. 2017). These characteristics of ERQs match our model assumptions, offering a unique test for the validity of our reddened templates.

Hamann et al. (2017) built the median SEDs of type-1 ERQs and showed that they were inconsistent with the simple reddening of the UV-optical SED of the normal quasar template, assuming an SMC-like extinction curve (see their Section 5.5). In Figure 12, we compare their median ERQ SED for the non-BAL core sample with our model templates used for low- $z$ Seyfert-1 nuclei. Without any fine-tuning of the dust geometry and grain properties, this composite SED can be matched by the reddened normal AGN template with $\tau_{\mathrm{V}}=3.0$.

The success of our model for these ERQs indicates that similar dust obscuration structures as well as the grain properties of polar dust might be shared among AGNs with a very wide range of luminosities $\left(L_{\mathrm{AGN}, \text { bol }} \sim 10^{8}-10^{13} L_{\odot}\right)$. 


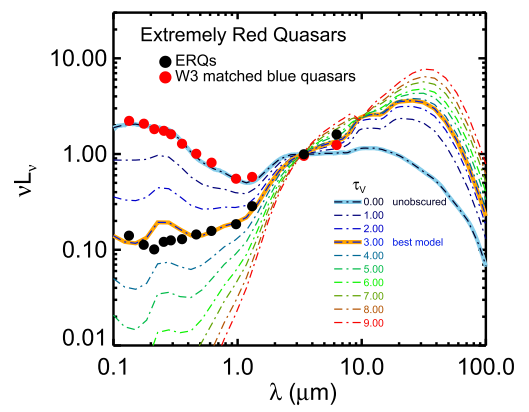

Figure 12. Reproduction of the median SEDs of ERQs and blue quasars matched to the ERQ sample in the WISE W3 bands from Hamann et al. (2017). The polar dust component is configured with the same parameters as NGC 3783. The ERQ SED is best matched by the reddened normal AGN template with $\tau_{\mathrm{V}} \sim 3.0$.

\subsection{AGNs with Mid-IR Warm-excess Emission}

From a study of $24 \mu \mathrm{m}$ selected AGNs in the Local Cluster Substructure Survey, Xu et al. (2015) showed that the UV to IR SEDs of most type-1 AGNs at $z \sim 0.3-2.5$ can be reasonably reproduced by combining three empirical templates that describe AGN, stellar, and star formation components. However, an additional warm dust emission component $(T \gtrsim 50 \mathrm{~K})$ was found for eight type-1 AGNs whose SEDs cannot be fitted by combining the Elvis-like intrinsic AGN template and any SFG galaxy template. Similar SED behavior has also been reported in other high- $z$ samples (e.g., Kirkpatrick et al. 2015) as well as a few quasars in the PG sample (Lyu et al. 2017).

Figure 13 shows an example of high- $z$ warm-excess AGNs in $\mathrm{Xu}$ et al. (2015). The contribution of the warm-excess emission to the total IR luminosity of this object is estimated to be $56 \%$ using the original Elvis-like AGN template (Xu et al. 2015) and the Rieke et al. (2009) SFG template. As demonstrated in this work, warm-excess emission above the intrinsic AGN templates can be easily produced if there is polar dust around the nucleus. After allowing the AGN template to be obscured by our model, we made excellent fittings of the rest frame $0.1-500 \mu \mathrm{m}$ SEDs for all the warm-excess AGNs reported in $\mathrm{Xu}$ et al. (2015).

The real origin of the warm-excess emission is not clear. $\mathrm{Xu}$ et al. (2015) demonstrated that this warm component can be fitted by the spectrum of a parsec-scale starburst disk (Thompson et al. 2005; Ballantyne 2008). In contrast, the success of our reddened AGN model suggests that this feature could be caused by the AGN heating of the surrounding polar dust, a possibly common phenomenon in many objects.

\subsection{Hot Dust-obscured Galaxies}

An important discovery made by the WISE survey is the identification of very luminous hot DOGs (Eisenhardt et al. 2012; Wu et al. 2012). Compared with typical IR luminous galaxies, the SEDs of hot DOGs have very prominent hot dust emission with characteristic temperatures around 60-100K (e.g., Wu et al. 2012; Fan et al. 2016). From investigations of X-ray observations and rest-frame optical spectra, these objects are found to be powered by luminous but heavily reddened type-1 AGNs (e.g., Stern et al. 2014; Ricci et al. 2017a; Wu et al. 2018).
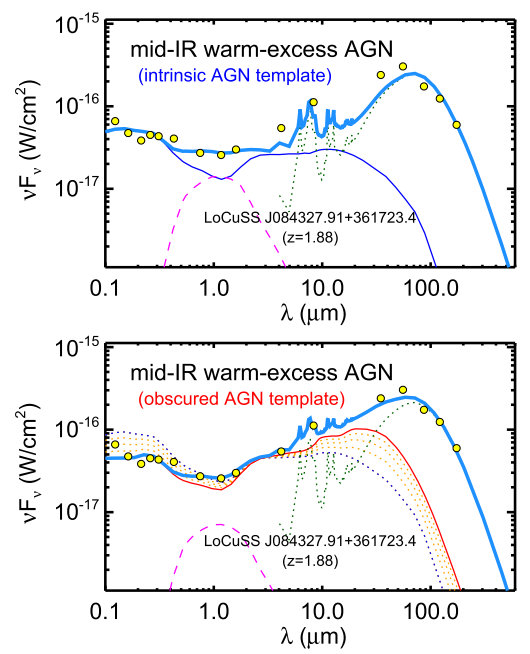

Figure 13. Best-fit results for a $z=1.883$ type-1 AGN, LIRAS J084327.91 +361723.4 , in Xu et al. (2015). Top: the results from fitting the SED with the intrinsic normal AGN template (blue solid thin line), the stellar template (magenta dashed line) and the star-forming template (green dotted line); the observed SED (yellow points) presents significant warm-excess emission in the mid-IR and far-IR compared with the final best-fit model (sky blue solid thick line). Bottom: the same fit as above, but with the addition of a standard template for emission by polar dust (red solid line).

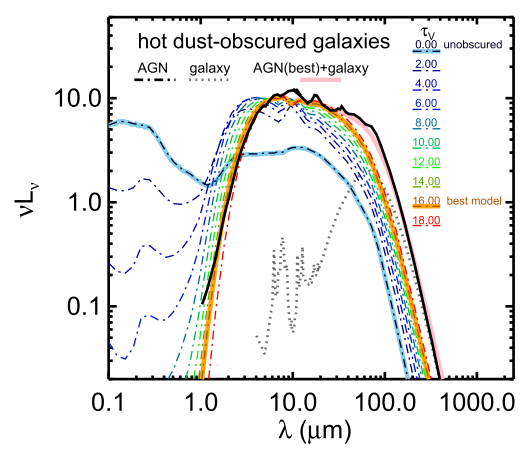

Figure 14. Reproduction of the median SED of HDO AGNs from Fan et al. (2016). This SED is roughly matched with the reddened AGN template with $\tau_{\mathrm{V}} \sim 16.0$ (orange thick line) plus a moderate star formation template (gray dotted line) in the far-IR. Compared with other objects, the polar dust geometry to reproduced the hot DOG SEDs features a steeply decreasing density profile $\left(n \sim r^{-1.5}\right)$ and more extended structure $(Y \gtrsim 1000)$.

Fan et al. (2016) presented the IR SEDs of 22 submmdetected hot DOGs and suggested that they can be described by the CLUMPY torus model (Nenkova et al. 2008a, 2008b) plus a cold dust component to represent the host galaxy star formation. Given the large number of free parameters of their models, we would like to see if our semi-empirical templates can provide an alternative solution.

Figure 14 presents a median IR SED of hot DOGs (Fan et al. 2016). To represent the galaxy far-IR emission, we adopted the empirical SFG SED library derived by Rieke et al. (2009). Since the reddened AGN templates trained for NGC 3783 do not contain such strong hot dust emission, we allowed the geometry of the extended dust component to be variable but left the dust grain properties unchanged. Similar to the case for NGC 3783 (see Section 2.4), we used the MCMC algorithms to find the best-fit parameters. The results are summarized in Table 5. Reasonable fittings can be achieved 

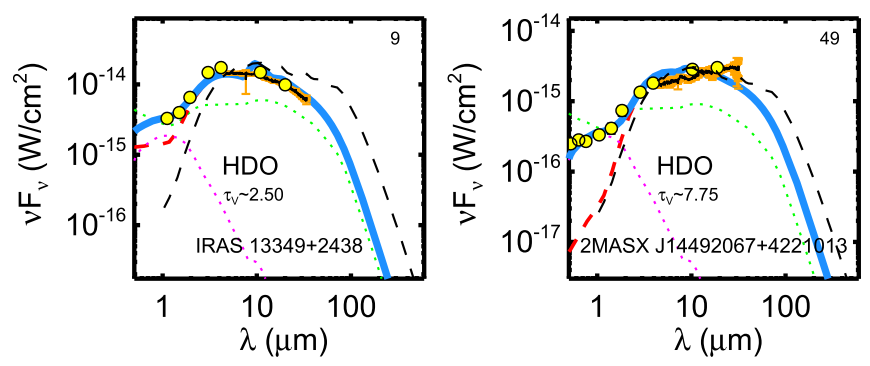

Figure 15. SEDs and the best-fit models of low- $z$ candidate HDO AGNs. The legend follows Figure 5. In each panel, we plot the composite SED template for hot DOGs from Fan et al. (2016) as a black dashed line.

Table 5

Suggested Model Parameters for Hot DOGs

\begin{tabular}{lclc}
\hline \hline Parameter & Label & Adopted Value & MCMC output \\
\hline$r_{\text {in }}$ temperature & $T_{\text {in }}$ & $\mathbf{1 5 0 0 ~ K}$ & $\ldots$ \\
Density profile & $\alpha$ & 0.50 & $1.49_{-0.45}^{+0.46}$ \\
Outer-to-inner radius & $Y$ & 5000 & $4778_{-3323}^{+4762}$ \\
Silicate:graphite mixture & $\ldots$ & $\mathbf{0 . 5 3 : 0 . 4 7}$ & $\ldots$ \\
Maximum grain size & $a_{\max }$ & $\mathbf{1 0} \mu \mathrm{m}$ & $\ldots$ \\
Minimum grain size & $a_{\min }$ & $\mathbf{0 . 0 4} \mu \mathrm{m}$ & $\ldots$ \\
\hline Input radiation SED & $\ldots$ & $\mathbf{n o r m a l}$ & $\ldots$ \\
Optical depth & $\tau_{\mathrm{V}}$ & 16 & $17.16_{-1.97}^{+1.64}$ \\
$\log \left(L_{\mathrm{SF}, \text { temp }} / L_{\odot}\right)$ & $\ldots$ & 11.5 & $11.49_{-0.44}^{+0.46}$ \\
\hline
\end{tabular}

Note. We use boldface to indicate assumed parameter values that do not go to MCMC parameter space sampling.

with a broad range of parameters, such as a dust density profile $r^{-1.5}$, and outer-to-inner radius $Y=5000$. Compared with most Seyfert nuclei, the fitted polar dust component for AGNs in hot DOGs has a steeper density profile $\left(n \propto r^{-0.5} \rightarrow r^{-1.5}\right)$ and more extended distribution $(Y \sim 500 \rightarrow 5000)$.

Are the best-fit parameters of our model to match the hot DOG SED physical? Assuming the central engine has $L_{\mathrm{AGN}, \text { bol }}=10^{14} L_{\odot}$, the suggested extended dust would extend to kpc scales. Interestingly, ALMA observations of [C II] emission in the most luminous hot DOG W2246-0526 show a uniform and highly turbulent ISM, suggesting isotropic galaxyscale outflows (Díaz-Santos et al. 2016), which is consistent with our argument that the extended dust distribution might be related to AGN outflows. Hot DOGs are suggested to live in overdense environments based on submm (Jones et al. 2014, 2015) and near-IR observations (Assef et al. 2015). It is possible that these objects represent a special phase of galaxy evolution, e.g., galaxy mergers, that could change the gas and dust distribution around the AGNs compared with the relatively undisturbed environment for typical Seyfert nuclei. Indeed, complex velocity structures based on $\mathrm{CO}(4-3)$ emission lines are reported in hot DOGs, suggesting a violent environment in such systems (Fan et al. 2018). Lastly, the SED model selected the Rieke et al. (2009) SFG template with $\log \left(L_{\mathrm{SF}, \text { temp }} / L_{\odot}\right) \sim$ 11.5, consistent with expectations for IR luminous galaxies at the hot DOG redshifts (Rujopakarn et al. 2013).

Although hot DOGs are claimed to exist only at high $z$ (e.g., Eisenhardt et al. 2012; Wu et al. 2012), type-1 AGNs with similar SED features could exist at low $z$. In Section 3.3, we note that two low- $z$ Seyfert-1 nuclei, IRAS $13349+2438$ $(z=0.11)$ and 2MASX J14492067+4221013 $(z=0.18)$, present strong hot dust emission for which our model with parameters set for NGC 3783 did not produce reasonable fits. In Figure 15, we present the SEDs of these objects and the bestfit model with the reddened AGN templates developed for hot DOGs. Due to their relatively low AGN luminosities, the stellar contamination in the near-IR cannot be ignored so that the apparent hot dust emission is not as strong as the case of high- $z$ hot DOGs. However, these objects have much stronger hot dust emission compared with the normal AGN template. Together with the stellar template, our HDO AGN templates explain these SEDs well. Thus, we suggest AGNs with some extreme SEDs similar to high- $z$ hot DOGs do exist at low $z$.

\section{Discussion}

We have shown that the IR SEDs of most type-1 AGNs regardless of luminosity can be fitted by a small set of templates for the accretion disk and torus, supplemented by an extended component to represent emission by polar dust. This result indicates that the differences in the SEDs between Seyfert-1 nuclei and type-1 quasars are largely the result of the absorption of UV energy by polar dust and its reradiation in the IR. As argued by, e.g., Stalevski et al. (2016), the IR SEDs of the optically thick torus would stay almost the same for any lines of sight not blocked by the torus. Our intrinsic AGN templates should be faithful descriptions of the torus emission in type-1 systems with a range of possible observing angles. The suggested polar dust for most objects can be modeled with the similar density profiles and grain properties. Therefore, the diverse broad-band IR SED features of many type-1 AGNs are only determined by two free parameters: the AGN intrinsic IR SED type and the level of surrounding obscuration. These results have the following implications.

\subsection{Interpreting the AGN Mid-IR Emission}

Previous AGN-related studies mostly only consider the effects of the extinction in the UV-optical bands, leaving the IR SED unchanged (e.g., Assef et al. 2010; Xu et al. 2015). An embedded assumption for such an approach is that the dust is distributed very far away from the AGN and the reprocessed emission is weak and possibly mixed with the host galaxy emission heated by stars. However, as suggested in this work, if the obscuration occurs in the vicinity of the AGN, it could naturally explain the polar dust emission in nearby AGNs as a result of energy balance and reconcile the diverse broad-band IR SED features of various AGN populations. This IRreprocessed emission can be an important contributor to the AGN-heated IR features besides the torus.

Various radiative transfer models have been proposed to reproduce the AGN IR emission. For simplicity, the geometric structure of the nuclear dust is commonly assumed to be doughnut-like and numerous dusty torus radiative transfer models have been developed to produce the AGN-heated IR SEDs (e.g., Fritz et al. 2006; Nenkova et al. 2008a; AlonsoHerrero et al. 2011; Stalevski et al. 2012; Siebenmorgen et al. 2015). Although they have matched the SEDs or spectral observations (e.g., Fritz et al. 2006; Nenkova et al. 2008b; Alonso-Herrero et al. 2011; Siebenmorgen et al. 2015), most such comparisons are limited to the mid-IR bands and strong parameter degeneracy exists (e.g., see discussions in Hoenig 2013 and Netzer 2015). Additionally, some work found 
that the torus models alone are typically not enough to reproduce the complete AGN IR SEDs of bright quasars (e.g., Mor et al. 2009; Leipski et al. 2014).

An important support for the clumpy torus models comes from their ability to reproduce the behaviors of AGN mid-IR silicate features, especially the silicate emission seen in some type-2 AGNs (e.g., Nikutta et al. 2009, but see Feltre et al. 2012). However, the possible existence of AGN-heated polar dust makes the interpretation of the origin of the mid-IR spectral features ambiguous. In fact, as suggested by, e.g., Sturm et al. (2005), the silicate emission feature observed in Seyfert nuclei could come from low-optical-depth dust located in the AGN NLRs. The success of our model supports this possibility (also see, e.g., Efstathiou 2006; Schweitzer et al. 2008).

For a single object, the AGN-heated IR spectral features are a result of a mixture of intrinsic variations of the torus properties, possible IR-reprocessed emission by the polar dust, and some specific observing angle. With these complications as well as the torus model degeneracies, the fittings of individual observations provide little information on the credibility of the model. Instead, we suggest that the best test is to see if the torus model can reproduce the empirical unobscured AGN templates, such as the three in Lyu et al. (2017). The objects used to derive the templates do not have evidence of obscuration, so that (1) the possibility of the polar dust emission is minimized and (2) these templates represent a face-on view of the system. In addition, by constructing average templates, variations of individual objects are smoothed out. In other words, the model degeneracies and observational uncertainties are greatly reduced.

\subsection{Dust-covering Factor of Type-1 AGNs}

A common tool used to study the AGN dust environment is the so-called dust-covering factor, typically measured by the relative flux ratios between the near- to mid-IR band and the optical bands (e.g., Maiolino et al. 2007; Treister et al. 2008; Mor \& Trakhtenbrot 2011; Roseboom et al. 2013; Lusso et al. 2013). Many authors have tried to use this parameter to explore the possible evolution of the torus, assuming the AGN near- to mid-IR emission comes from the torus and the optical emission originates from the accretion disk (e.g., Lawrence 1991; Simpson 2005; Assef et al. 2013). Given the direct detections of the polar dust (Braatz et al. 1993; Cameron et al. 1993; Raban et al. 2009; Hönig et al. 2012, 2013) and its possibly frequent occurrence inferred in this work as well as, e.g., Asmus et al. (2016), we should be cautious about the interpretations of the AGN IR-to-optical flux ratios.

We can obtain a rough idea of how significantly the AGN IR-to-optical flux ratio can be changed by the polar dust. Figure 16 presents some characteristic tracers of dust-covering factors calculated from our reddened AGN model as a function of optical depth $\tau_{\mathrm{V}}$. For the direct observed values, we divided the IR luminosity, $L_{\mathrm{IR}}$, at $\lambda=3,6.7,15 \mu \mathrm{m}$ by the apparent AGN optical luminosity, $L_{\text {opt }}$, at $5100 \AA$ of reddened templates for normal, WDD, and HDD AGNs. In all cases, the IR-tooptical flux is found to increase exponentially with the optical depth. With moderate extinction $\left(\tau_{\mathrm{V}} \lesssim 1-2\right)$, the change caused by polar-dust obscuration and emission would easily exceed the intrinsic variations of the torus in a type-1 AGN. Conversely, if we use the intrinsic (unobscured) luminosity at $5100 \AA$ to represent the $L_{\text {opt }}$, the integrated optical depth of the

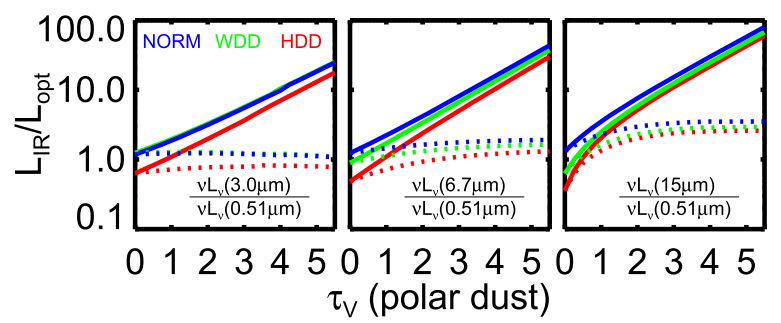

Figure 16. MIR-to-optical luminosity ratios $\left(L_{\mathrm{IR}} / L_{\mathrm{opt}}\right)$ as a function of optical depth of the extended dust component $\left(\tau_{\mathrm{V}}\right)$ from our model for normal (blue), WDD (green), and HDD (red) AGNs. We compute these relations with the apparent (obscured) and intrinsic (unobscured) values of $L_{\mathrm{opt}}$ and show them separately as solid and dotted lines.

polar dust component would have limited influence on $L_{\mathrm{IR}} / L_{\mathrm{opt}}$, especially at shorter wavelengths. With $\tau_{\mathrm{V}}$ ranges from $0-5$, the values of $L_{3.4 \mu \mathrm{m}} / L_{0.51 \mu \mathrm{m}}$ are changed by a factor of $0.94,0.91$, and 1.26 for normal, WDD and HDD AGNs. For $L_{6.7 \mu \mathrm{m}} / L_{0.51 \mu \mathrm{m}}$, the changes are $1.53,1.81$, and 2.67. For $L_{15 \mu \mathrm{m}} / L_{0.51 \mu \mathrm{m}}$, the corresponding values are $2.68,4.67$, and 7.28. Since the SED of polar dust emission peaks at $\lambda \sim 26 \mu \mathrm{m}$ (Section 4), its effects would be only important for the dust-covering factors of warm and cold dust.

Nevertheless, as discussed in Section 2.2.2, the optically thin IR emission from a dust component with different dustcovering factors can be easily reproduced by changing its overall optical depth $\tau_{\mathrm{V}}$ with an assumption of a spherical dust distribution. Given this degeneracy, it is impossible to get a definite estimation of the dust geometry, like its covering factor, from the SED alone when the IR emission is not optically thick.

Besides the possible existence of polar dust, there are many other factors that can change the value of $L_{\mathrm{IR}} / L_{\mathrm{opt}}$. In the optical band, the AGN emission can be modified by the host galaxy contamination, dust obscuration at different physical scales with uncertain extinction curves, or even some shortterm variability. Based on a 3D radiative transfer model, Stalevski et al. (2016) explored how the anisotropic emission of the dusty torus and the accretion disk could influence the estimation of real dust-covering factor from $L_{\mathrm{IR}} / L_{\mathrm{opt}}$, showing that their relation could be nonlinear and depend strongly on the assumed torus optical depth. Considering these complications, it is difficult to make conclusive arguments on the meaning of $L_{\mathrm{IR}} / L_{\mathrm{opt}}$ traced by the simple colors derived from a few photometric bands.

\subsection{AGN X-Ray Obscuration and Polar Dust Optical Depth}

The extinction caused by the polar dust component in many AGNs is likely associated with the behavior of their X-ray obscuration. To explore this possibility, we collected the literature measurements of the absorbing column density, $N_{\mathrm{H}}$, and compared them to the derived integrated optical depth of the polar dust component (derived purely from IR SED fittings) in Figure 17. Forty-one out of 64 nearby Seyfert-1 nuclei in our sample are found to have good measurements. Among them, besides 3C 219 (taken from Comastri et al. 2003) and Mrk 1239 (taken from from Corral et al. 2011), the measurements of all others are collected from Ricci et al. (2017b).

First we check if the X-ray obscuration and the possible optical extinction caused by polar dust are consistent. As argued by Shimizu et al. (2018), most type-1 AGNs are 


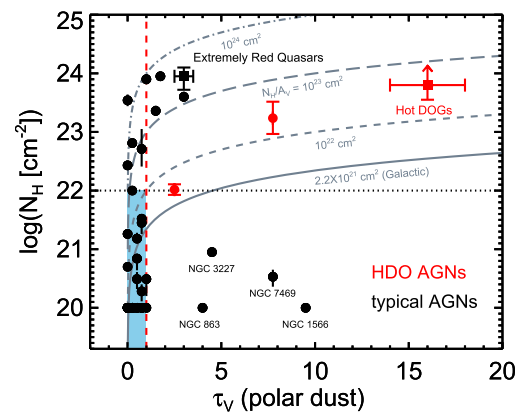

Figure 17. The distribution of gas column density and polar dust optical depth of the low $-z$ Seyfert- 1 (dots) and the high- $z$ reddened populations (squares). We use the black color to indicate objects whose IR SEDs are best described by the NGC 3783-like model and red colors to indicate the HDO AGNs. The blue shaded region represents low-extinction type-1 AGNs with $A_{\mathrm{V}} \lesssim 1$ mag and $N_{\mathrm{H}} \lesssim 10^{22} \mathrm{~cm}^{2}$

unobscured in the X-ray, i.e., $N_{\mathrm{H}} \lesssim 10^{22} \mathrm{~cm}^{-2}$. If we adopt the AGN value of $N_{\mathrm{H}} / A_{\mathrm{V}}$ from Maiolino et al. (2001b), this means the corresponding optical extinction should satisfy $A_{\mathrm{V}} \lesssim 1$. By this criteria, 28 out of the 41 objects $(\sim 68 \%)$ have low extinction both in the X-ray and optical. In addition, 9 out of $41(\sim 22 \%)$ are X-ray absorbed without much polar dust extinction. For most X-ray obscured Seyfert-1 nuclei for which the NGC 3783-like model works well, a value of $N_{\mathrm{H}} / A_{\mathrm{V}} \gtrsim$ $10^{23} \mathrm{~cm}^{-2} / \mathrm{mag}$ seems favored, which is much higher than the typical values found for the Galactic ISM (e.g., $\sim 2.2 \times$ $10^{21} \mathrm{~cm}^{-2} / \mathrm{mag}$; Güver \& Özel 2009). This nicely fits the picture that small grains, which are the most efficient carriers for the extinction in the optical band, can be easily destroyed by their direct exposure to AGN radiation. The effect of large grains agrees with the behavior of the nine X-ray-absorbed objects with small $A_{\mathrm{V}}$. Meanwhile, there are four outstanding outliers with high $\tau_{\mathrm{V}}$ but very low $N_{\mathrm{H}}$. However, this is also not a surprise since $\tau_{\mathrm{V}}$ describes the average polar dust optical depth along all possible LOSs. If the polar dusty clouds distribute close to the edge of the ionization core or are simply clumpy, the AGN X-ray corona could be unobscured along some lucky observing angles while the amount of polar dust is still significant. Consequently, we conclude that there is no obvious tension between the literature results on the X-ray obscuration and the optical depth of the polar dust component inferred by our SED model.

We can also compare the behaviors of the high- $z$ ERQs and hot DOGs discussed in Section 5 with these low- $z$ Seyfert-1 nuclei to further check if they are also similar (or not) in the $\mathrm{X}$-ray. Taking the gas column density measured for the stacked X-ray images from Goulding et al. (2018), a typical value of $N_{\mathrm{H}} / A_{\mathrm{V}}$ for ERQs is $\sim 3 \times 10^{23} \mathrm{~cm}^{-2} \mathrm{mag}^{-1}$, consistent with the trend of most X-ray obscured Seyfert-1 AGNs in our sample. In other words, besides a polar dust configuration resembling NGC 3783, these high- $z$ ERQs also share the relation between X-ray gas obscuration and optical dust extinction of typical reddened Seyfert-1 nuclei.

In contrast, hot DOGs might be different. We computed an average $N_{\mathrm{H}} \gtrsim 6 \times 10^{23} \mathrm{~cm}^{-2}$ for high- $z$ hot DOGs with reported measurements from the literature (see Vito et al. 2018 and references therein). Combined with the optical depth of polar dust component estimated from our SED modeling, this suggests a $N_{\mathrm{H}} / A_{\mathrm{V}} \gtrsim 4.0 \times 10^{22} \mathrm{~cm}^{-2}$. Together with the measurements of two low $-z$ candidates, these HDO objects have $N_{\mathrm{H}} / A_{\mathrm{V}}$ values of $\sim 10^{22}-10^{23} \mathrm{~cm}^{-2} / \mathrm{mag}$, which are lower than those of typical Seyfert-1 nuclei (as well as the ERQs) but still higher than the galactic values. Perhaps the hot DOGs do represent a different phase of AGN-host evolution compared to typical Seyfert-1 nuclei (as well as ERQs) so that their polar dust grains are not removed as significantly as the latter. For example, the host galaxies of HDO AGNs could be very obscured so that the AGN radiation is not efficient enough to destroy most dust distant from the nucleus. Alternatively, considering the large extent of the outflows in these cases, there could be some channels for dust production, e.g., (post-)AGB stars and/or supernovas, that mitigate the effects of dust destruction by the AGN.

\subsection{AGN Structures and Unification}

An anisotropic obscuration structure composed of optically thick dust, typically pictured as a torus, provides a simple solution to unify the behaviors of different types of AGNs (Antonucci 1993; Urry \& Padovani 1995). Besides the optically thick torus, this work suggests the presence of an extended dust distribution that modifies both UV-optical and IR properties of the AGN. We can get some rough ideas on the physical scales associated with the extended dust distribution and discuss its relation with other known AGN components.

Based on our results, the observations of many type-1 AGNs can be reproduced by assuming the same polar dust model configuration featuring a density profile $r^{-0.5}$, outer-to-inner radius $Y=500$, and temperature at the inner boundary $T \sim$ $1500 \mathrm{~K}$. For an $L_{\odot}=10^{11} \mathrm{AGN}$, the dust responsible for the extended obscuration has a maximum size $r_{\text {out }} \sim 0.1 \mathrm{kpc}$, which is about one order of magnitude larger than the size of cold dust in the AGN torus ( $r_{\text {torus,cold }} \sim 10 \mathrm{pc}$; see Section 4.2 in Lyu \& Rieke 2017). AGN-driven winds or outflows can easily distribute dust around the torus to such scales.

One likely location for the polar dust grains is the NLR clouds. In fact, the required $A_{\mathrm{V}} \lesssim 5$ mag for most type-1 AGNs can be easily reproduced by combining the typical NLR column density $\left(N_{\mathrm{H}} \sim 10^{20}-10^{21} \mathrm{~cm}^{-2}\right)$ with the assumption of the classical value of $N_{\mathrm{H}} / A_{V} \sim 2.2 \times 10^{21} \mathrm{~cm}^{-2} / \mathrm{mag}$ in the galactic ISM (Güver \& Özel 2009). Alternatively, if we adopt $N_{\mathrm{H}} / A_{V} \gtrsim 10^{22} \mathrm{~cm}^{-2} / \mathrm{mag}$ for Seyfert galaxies (Maiolino et al. $2001 \mathrm{~b}$ ) and assume the NLR cloud density of $\sim 100 \mathrm{~cm}^{-3}$, the required physical scale of the LOS dusty clouds would span $\sim 150 \mathrm{pc}$, which is also realistic and consistent with the value estimated above.

In Figure 18, we illustrate the various dust components surrounding a typical Seyfert nucleus. Very close to the accretion disk, dust could not survive due to evaporation at high temperatures. Since the accretion disk emission is not isotropic, some concave shape of dust-free regions is expected above and below the accretion disk. In the equatorial direction, we expect a torus-like component. Thanks to the shielding against the direct AGN emission by very optically thick $\left(\tau_{\mathrm{V}} \gtrsim 20-50\right)$ clouds, a range of dust grain properties is expected within the torus. Along the polar direction, as discussed in Section 2.2.1, only large dust grains might survive. In other words, we would expect the dust properties are changing along different observing angles (see Baskin \& Laor 2018), which may lead to diverse AGN extinction curves as reported in the literature (e.g., Hall et al. 2002; Richards et al. 2003; Czerny et al. 2004; Gaskell et al. 2004; Hopkins et al. 2004; Gaskell \& Benker 2007). In regions far away from the nucleus, e.g., the galactic ISM at (sub-)kpc scales, the influence 


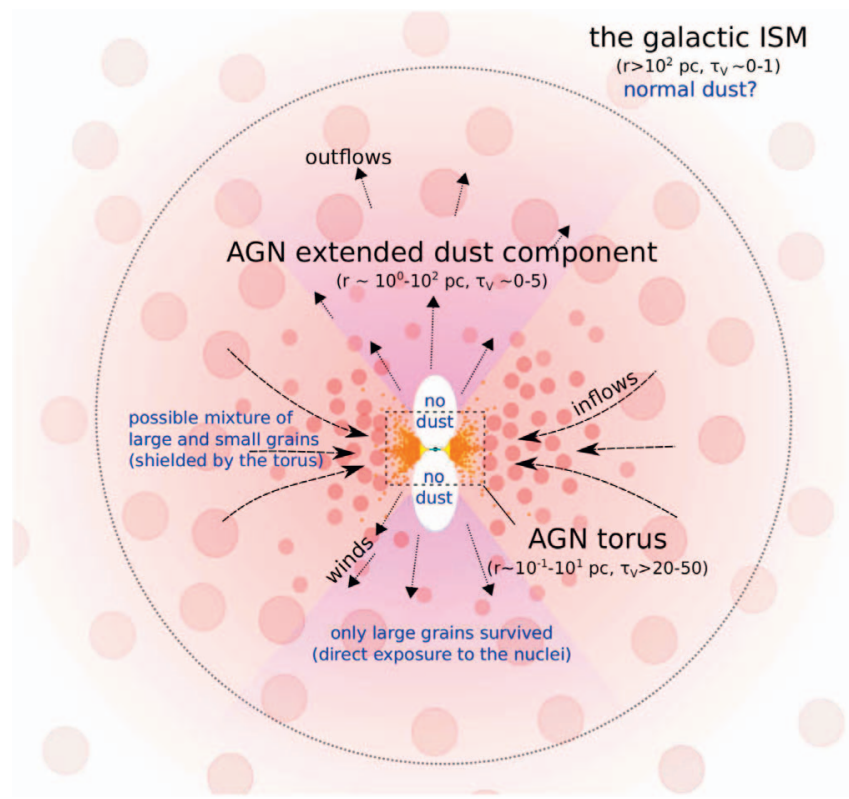

Figure 18. Schematic drawing for the dust environment around a typical Seyfert-like nuclei. See the text for details.

of the AGN is minimal so that classical dust properties are expected. Between the torus and the galactic ISM, there exists an extended dust component with low optical depth $\left(\tau_{\mathrm{V}} \lesssim 5\right)$, which causes the LOS obscuration and the polar dust emission seen in type-1 AGNs. In reality, there should be no clear boundaries between these components and they can exchange dust by various feedback mechanisms, e.g., inflows or outflows. Although our sketch shows a clumpy environment, the actual configuration could have other forms, such as filaments (Wada et al. 2009; Wada 2012). In addition, the shape of the polar dust distribution could deviate strongly from isotropic symmetry, e.g., having some dust-covering factor.

The intrinsic IR emission of $\mathrm{AGNs}$ at wide ranges of luminosity $\left(L_{\mathrm{AGN}, \mathrm{bol}} \sim 10^{8}-10^{14} L_{\odot}\right)$ and redshift $(z \sim 0-6)$ appears to be described well by a limited family of SED shapes. In Lyu et al. (2017), we demonstrated that the intrinsic IR emission of unobscured quasars can be represented by a similar set of templates independent of redshift. Based on the low- $z$ PG sample, complete IR SED templates have been built and tested for AGNs with $L_{\mathrm{AGN}, \text { bol }} \gtrsim 10^{11} L_{\odot}$. With the results obtained in this work, their validity is extended to Seyfert-1 nuclei and some peculiar AGN populations. Besides the torus SED variations, the IR SED differences are caused by an extended dust component that might reside in NLR clouds. Consequently, it is not necessary to invoke any new component that is only found in some special groups of AGNs.

\subsection{The Diverse Dust Environments of AGNs}

With our previous study of unobscured quasars (Lyu et al. 2017; Lyu \& Rieke 2017) and the results on other type-1 AGN populations obtained here, we have found that the IR properties of AGNs are not only determined by the observing angles, but also caused by the intrinsic variations of the torus and the different contributions of polar dust.
As illustrated in Figure 19, dust environments of AGNs can be grouped into three different scenarios, characterized by the obscuration along the face-on direction:

1. Unobscured AGNs: The IR properties of such systems are dominated by a torus-like structure without much dust distributed along the polar direction. It is likely that AGN activity is extremely strong, blowing out the dusty gas or simply destroying most dust along the polar direction. The photons from the central engine can be directly seen from a face-on observing angle. Typical examples are luminous blue quasars, where there is little extinction in the UV-optical bands.

Due to its relatively compact size, the behavior of the torus is mainly determined by accretion parameters. As shown in Lyu et al. (2017), the intrinsic IR SEDs of unobscured quasars present clear variations and might be related to different AGN properties (e.g., luminosity and Eddington ratios). Among Seyfert nuclei, we have also seen the appearance of both normal and dust-deficient AGNs, suggesting the torus structures among Seyfert-1 populations are not exactly the same.

2. Moderately obscured AGNs: Besides the torus, there exists an extended dust component $\left(r \sim 10^{2} \mathrm{pc}\right)$ with low optical depth $\left(\tau_{\mathrm{V}} \gtrsim 0-5\right)$, which could be AGN dusty outflows, winds, or dusty NLRs. Along the polar direction, the dust obscures the central engine, resulting in mid-IR excess emission and some moderate level of optical extinction if the observation is not made through some lucky dust-free LOS.

Most AGNs, i.e., those in Seyfert galaxies, will fall into this category. In relation to unobscured quasars, our SED analysis suggested that Seyfert nuclei frequently have strong polar dust emission, indicating an extended dusty component that can be heated by the central engine. In fact, there are observational suggestions that the AGN NLR could disappear at very high luminosities (Netzer et al. 2004). If we believe these NLR clouds are dusty, a decreasing frequency of AGNs with polar dust emission with increasing luminosity should be expected.

3. Highly obscured AGNs: The AGN-heated dust in such systems can be very extended $\left(r \sim 10^{3} \mathrm{pc}\right)$ with high optical depth $\left(\tau_{\mathrm{V}} \sim 5-20\right)$, which might be a result of, e.g., galaxy mergers. The gravitational torque of largescale interaction can bring a substantial amount of galactic ISM toward the nucleus which can be collide with the AGN outflows, resulting in a very turbulent environment that changes the circumnuclear dust density profile. These objects present clear evidence of UVoptical obscuration and strong near- to mid-IR dust emission. Typical examples are AGNs in hot DOGs.

In this picture, the torus is formed and maintained during the BH accretion (e.g., Hopkins et al. 2012 and references therein). An extended dust component surrounding the AGN nucleus naturally bridges the torus and the host galaxy ISM. Both AGN and galaxy feedback may modify the properties of this component, resulting in the changing obscuration behaviors at different phases of galaxy evolution as suggested by many previous studies (e.g., Sanders et al. 1988a, 1988b; King 2003; Hopkins et al. 2010, 2016). 
(a)

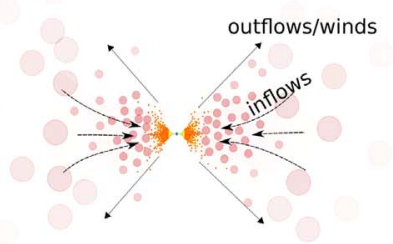

(b)

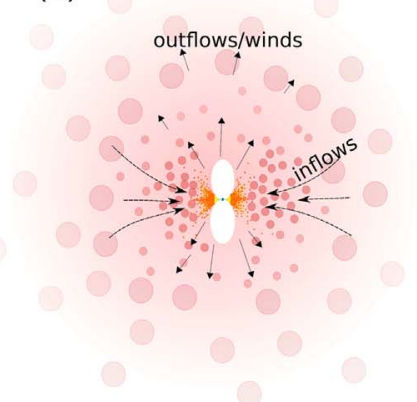

moderately obscured AGN (e.g., Seyfert nuclei)
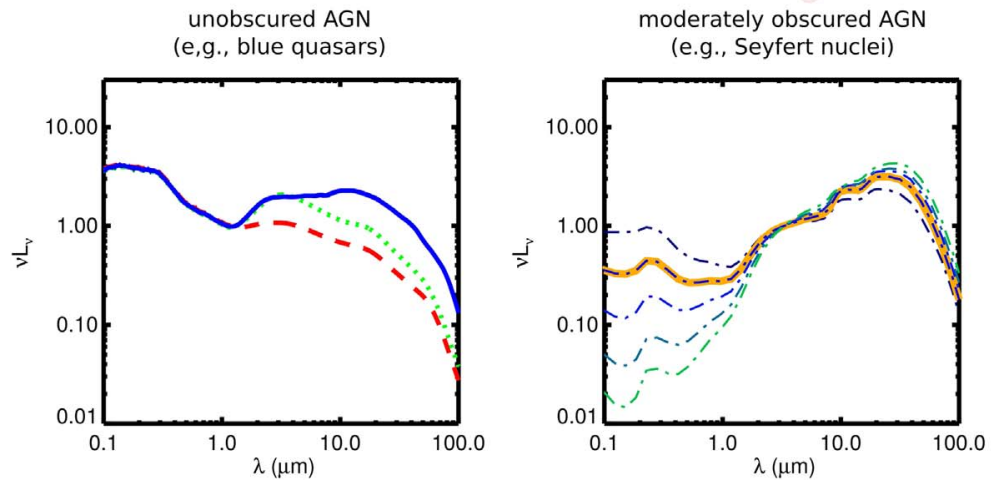
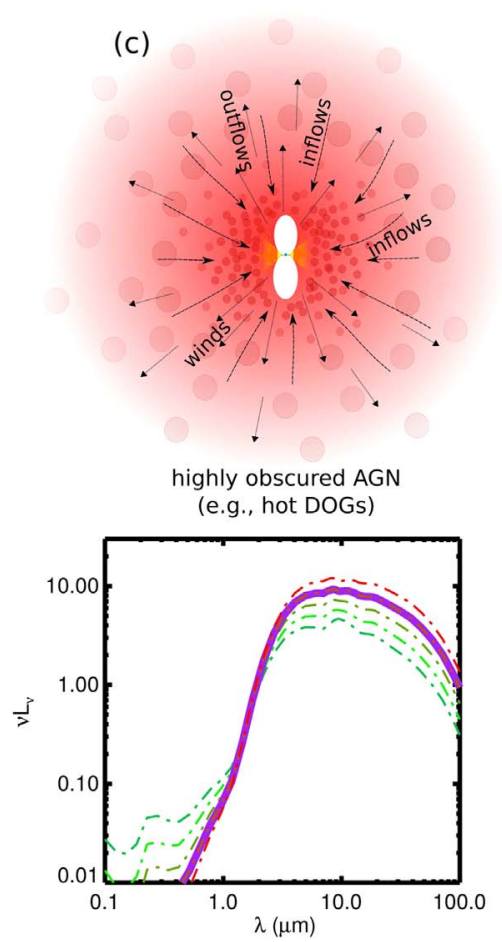

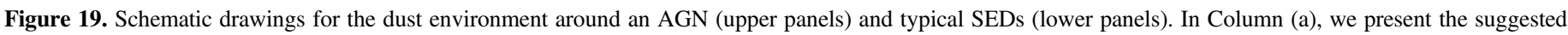

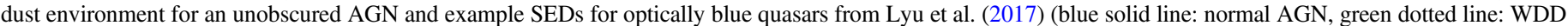

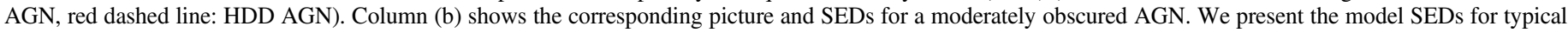

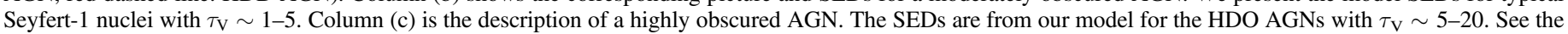
text for details.

\section{Summary and Conclusions}

Motivated by the commonly seen UV-optical obscuration and the discoveries of polar dust emission in a few nearby Seyfert nuclei, we have proposed an empirically driven model to produce the SEDs of reddened type-1 AGNs. The intrinsic AGN templates in Lyu et al. (2017) have been assumed as reasonable choices for polar-dust-free type-1 AGNs. With radiation transfer calculations, we obscured them by an extended dusty structure with a power-law density profile and large dust grains. This model naturally:

1. reproduced the nuclear UV to mid-IR SED (Prieto et al. 2010) and polar dust emission strength (Hönig et al. 2013) for the type-1 AGN in NGC 3783;

2. fitted the IR SEDs of a large sample of low- $z$ Seyfert-1 nuclei with the mid-IR emission dominated by the AGN;

3. reproduced the UV to mid-IR composite SEDs of the SDSS/Spitzer type-1 AGNs;

4. matched the composite SED of extremely red luminous quasars in Hamann et al. (2017);

5. explained the warm-excess dust emission seen in some high- $z$ type-1 AGNs, as first reported by Xu et al. (2015);

6. reproduced the IR emission template of HDO AGNs proposed by Fan et al. (2016).

Our main conclusions are as follows:

1. The broad-band IR SEDs of low-z Seyfert-1 nuclei and some high- $z$ type-1 AGNs with peculiar SED features can be reconciled with the quasar intrinsic AGN templates by adding the IR-reprocessed emission of polar dust. It is possible that AGNs over a broad range of redshift and luminosity have a similar dust environment that features a circumnuclear torus plus an extended dust component.

2. The polar dust emission could be a natural result of the commonly seen UV-optical obscuration among Seyfert-1 nuclei. This statement is supported by our detailed SED analysis of NGC 3783 and the our successful fitting of the composite SEDs of the SDSS/Spitzer Seyfert-1 AGN sample. Surprisingly, we found the same radial density profile $\left(n \propto r^{-0.5}, r_{\text {out }} / r_{\text {in }} \sim 500\right)$ and dust grain properties $\left(a_{\max }=10 \mu \mathrm{m}, a_{\min }=0.04 \mu \mathrm{m}\right)$ of the extended dust component works for most Seyfert-1 nuclei.

3. The primary factor to determine the behavior of the polar dust emission is the overall optical depth. The emission SED of the AGN polar dust component peaks around $26 \mu \mathrm{m}$ with a characteristic temperature at $T \sim 113 \mathrm{~K}$, leaving strong mid- to far-IR warm-excess emission signatures above intrinsic AGN templates for some objects. The observed size of polar dust emission is a function of wavelength.

4. Our SED analysis provides an effective method to search for AGNs with polar dust emission. Among the 64 Seyfert- 1 nuclei studied in this work, we found that $\sim 1 / 3$ of them have significant polar dust emission that contributes at least half of the AGN emission at $\sim 10 \mu \mathrm{m}$, about another $1 / 3$ shows no evidence with IR SEDs matched by the intrinsic templates, and the remaining $1 / 3$ has weaker or absent polar dust emission -it is ambiguous.

5. The reddened type-1 AGN model trained for NGC 3783 not only fitted the SEDs of most Seyfert-1 nuclei but also worked reasonably well to reproduce the SEDs for high- $z$ 
ERQs and type-1 AGNs with mid-IR warm excess. These results suggest that most AGNs could share similar properties of extended dust environment, which may indicate that AGN-driven outflows dominate the largescale structure and grain properties of the nuclear extended dust environment.

6. The reddened type-1 AGN model can also reproduce the SEDs of hot DOGs when combined with an SFG template. However, the best-fit parameters suggest a more extended AGN-heated dust distribution with a steeper density profile compared with objects like NGC 3782. This indicates that the AGN dust environment in hot DOGs might be quite different from other systems. Objects with similar hot DOG SED features are also found at $z \sim 0.1$.

7. The general success of our reddened type-1 AGN model suggests the IR-reprocessed emission of the extended dust distribution in some objects can complicate the interpretation of the integrated AGN IR emission, such as the behavior of the torus radiative transfer models and constraints on their dust-covering factors.

8. The SED shape of the AGN thermal IR emission might be a reflection of its dust environment. Depending on the obscuration level along the face-on direction, we propose three basic scenarios, in which the AGN and galaxy feedbacks may play different roles, that lead to the diverse IR SED properties among type-1 AGNs.

From a technical perspective, contrasting with other alternatives to fit the AGN-heated dust emission in the literature, the semi-empirical model proposed here is able to fit a wide range of AGN SEDs using relatively few free parameters. Combined with well-constrained empirical templates of star-forming galaxies, we can get reasonably accurate separations of the AGNs and their host galaxies. Meanwhile, these observationally calibrated semi-empirical templates are a valuable tool to characterize the IR color space of AGNs, which could be useful to look for abnormal objects in the era of JWST.

We thank Dr. Aigen Li and Dr. Robert Nikutta for helpful discussions, Dr. Lei Hao for providing the spectral measurements of the SDSS DR7 Main Galaxy Sample, and Dr. Almudena Prieto for the clarification of NGC 3783 SED data. We are also grateful to the anonymous referee for a very constructive report and helpful comments.

This work was supported by NASA grants NNX13AD82G and 1255094 .

This work is based in part on observations made with the Spitzer Space Telescope, which is operated by the Jet Propulsion Laboratory, California Institute of Technology under a contract with NASA. The Combined Atlas of Sources with Spitzer IRS Spectra (CASSIS) is a product of the IRS instrument team, supported by NASA and JPL.

This publication has made use of data products from the Wide-field Infrared Survey Explorer, which is a joint project of the University of California, Los Angeles, and the Jet Propulsion Laboratory/California Institute of Technology, funded by the National Aeronautics and Space Administration. This publication also makes use of data products from NEOWISE, which is a project of the Jet Propulsion Laboratory/California Institute of Technology, funded by the Planetary Science Division of the National Aeronautics and
Space Administration. This publication makes use of data products from the 2MASS, which is a joint project of the University of Massachusetts and the Infrared Processing and Analysis Center/California Institute of Technology, funded by the National Aeronautics and Space Administration and the National Science Foundation.

Funding for the SDSS and SDSS-II has been provided by the Alfred P. Sloan Foundation, the Participating Institutions, the National Science Foundation, the U.S. Department of Energy, the National Aeronautics and Space Administration, the Japanese Monbukagakusho, the Max Planck Society, and the Higher Education Funding Council for England. The SDSS website is http://www.sdss.org/. The SDSS is managed by the Astrophysical Research Consortium for the Participating Institutions. The Participating Institutions are the American Museum of Natural History, Astrophysical Institute Potsdam, University of Basel, University of Cambridge, Case Western Reserve University, University of Chicago, Drexel University, Fermilab, the Institute for Advanced Study, the Japan Participation Group, Johns Hopkins University, the Joint Institute for Nuclear Astrophysics, the Kavli Institute for Particle Astrophysics and Cosmology, the Korean Scientist Group, the Chinese Academy of Sciences (LAMOST), Los Alamos National Laboratory, the Max-Planck-Institute for Astronomy (MPIA), the Max-Planck-Institute for Astrophysics (MPA), New Mexico State University, Ohio State University, University of Pittsburgh, University of Portsmouth, Princeton University, the United States Naval Observatory, and the University of Washington.

We acknowledge the use of the NASA/IPAC Extragalactic Database (NED), which is operated by the Jet Propulsion Laboratory, California Institute of Technology, under contract with the National Aeronautics and Space Administration. This work has also made use of the VizieR catalogue access tool, CDS, Strasbourg, France.

Software: DUSTY (Ivezic et al. 2017), SKIRT (Baes et al. 2003, 2011).

\section{Appendix A \\ The Effects of Optical Thickness}

We use 3D radiative transfer simulations to demonstrate how the optical thickness of the dusty medium can affect its SED. Detailed simulation and analysis of the IR emission from clumpy clouds is described in the literature (e.g., Nenkova et al. 2008a; Stalevski et al. 2012). We illustrate several key concepts focusing on a single dusty cloud heated by the emission from an accretion disk at some distance.

For simplicity, the cloud is assumed to be a sphere with a radius $r=1 \mathrm{pc}$ and a homogeneous density distribution of typical ISM dust grains. The accretion disk is approximated as a point source with a broken power-law SED, following Stalevski et al. (2016), and located at a distance of 5 pc to the cloud. We use the radiative transfer code SKIRT (Baes et al. 2003, 2011) to compute the output SEDs as well as the images observed from different angles as a function of the optical thickness of the cloud $\left(\tau_{\mathrm{cl}}\right)$. The results can be found in Figure 20 . If the cloud is optically thin $\left(\tau_{\mathrm{cl}} \lesssim 1\right)$, the IR SEDs would be identical for any viewing angles. In fact, if the V-band optical thickness is not very large $\left(\tau_{\mathrm{cl}} \lesssim 10\right)$, the cloud would be almost transparent for its own emission, given the rapidly decreasing extinction at longer wavelengths. These conclusions can be seen from the differential images between 

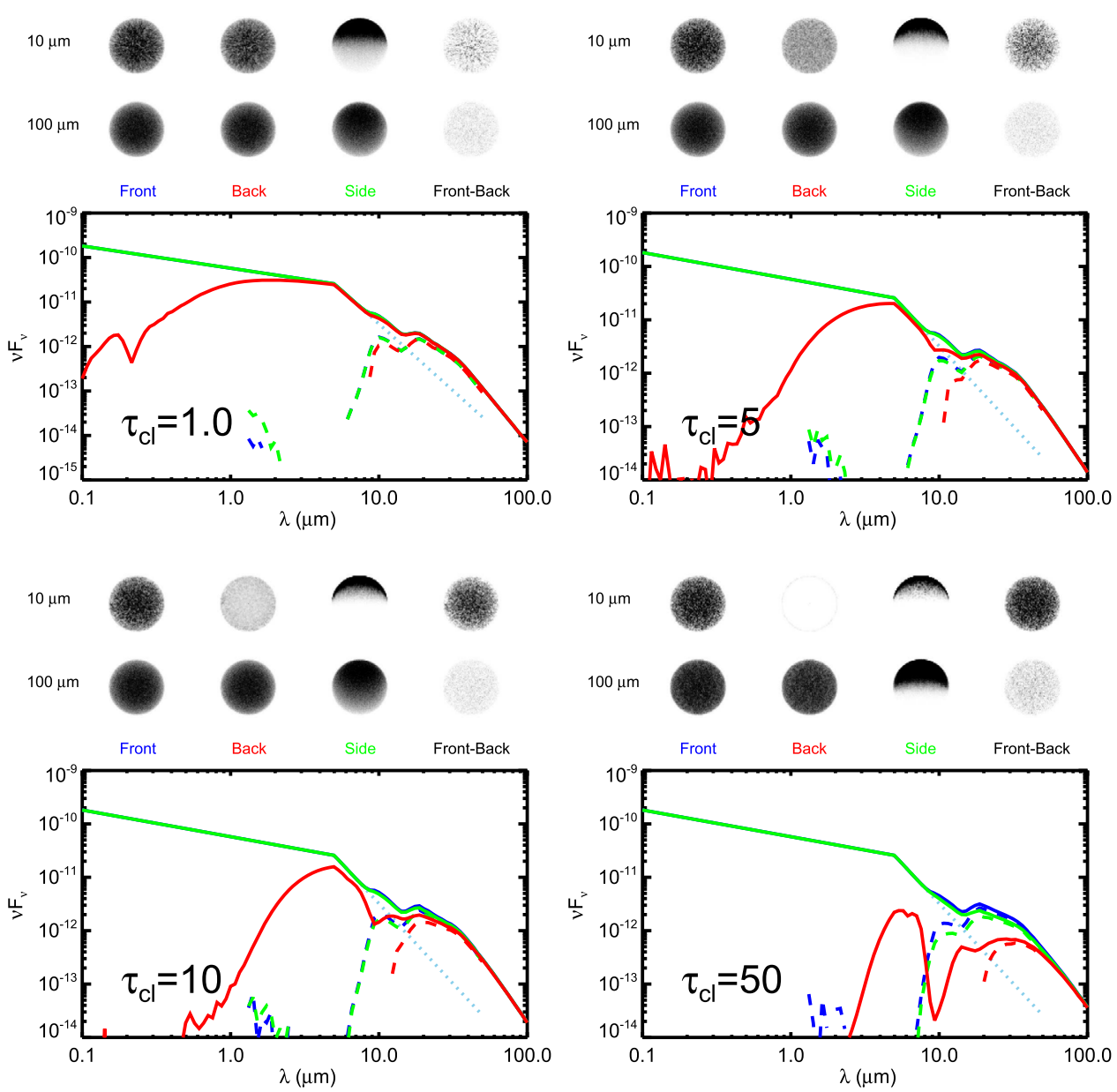

Figure 20. The output SEDs and images of a single dusty cloud plus the accretion disk emission viewing from different angles. The integrated SEDs are plotted as solid lines. The accretion disk emission is represented as the blue dotted line. We also show the pseudo-IR emission SEDs (dashed lines) of the cloud by subtracting the accretion disk SED from the total SED. We show the model images of the dusty cloud at 10 and $100 \mu \mathrm{m}$. The brightness is linearly scaled with the darkness of the pixel. To demonstrate the transparency of the dust cloud, a differential image "front-back" is also made by subtracting the "back" emission from the "front" emission at corresponding pixels.

the front view of the bright side of the cloud and the back view of the dark side of the cloud. In the case of blocking, the dust emission SED will not change once the optical depths at the corresponding wavelength are low (e.g., $\lambda \sim 100 \mu \mathrm{m}$ ).

The optically thin assumption also makes the effects of the dust-covering factor relatively easy to be accounted. In Figure 21, we symmetrically distribute spherical dusty spheres at the same distance and calculate the output SEDs. The shape of the dust-processed SED is the same as the case of a single cloud, and the intensity can be matched by linearly scaling the emission from the single cloud.

For a low-optical-depth dust component, the output SEDs are not sensitive to the clumpiness of the dusty clouds. We demonstrate the effect of clumpiness in Figure 22. We first use the SKIRT code to produce the output SED of a $\tau_{\mathrm{V}}=1.5$ dusty sphere with density profile $n \sim r^{-0.5}, r_{\text {in }}=0.6 \mathrm{pc}$ and $r_{\text {out }}=300 \mathrm{pc}$, assuming the large dust grain distribution $a_{\max }=10 \mu \mathrm{m}, a_{\min }=0.04 \mu \mathrm{m}$. With the same total dust grain mass, we break the smooth geometry into 1000 randomly distributed spherical clumps with different size $R_{\mathrm{cl}}$, but following the same density profile. Depending on whether the LOS is blocked by the dust, the UV-optical SEDs present large variations for different viewing angles. However, the dust-reprocessed IR SEDs are nearly identical. We conclude the clumpiness would not influence the IR SED shapes.

In summary, we have demonstrated that optical depth up to $\tau_{\mathrm{V}} \lesssim 5$ does not strongly modify the infrared output of a cloud composed of classical ISM dust and directly heated by an AGN. For larger grains suggested for polar dust, such effects would be further minimized. At the same radius, the integrated IR SED of $N$ identical clouds, whatever their placement, can be matched by linearly scaling the emission SED of single cloud by a factor of $N$. Assuming modest optical depths, the output SED is also insensitive to the clumpiness of the dusty medium. Thus, the primary determination of the infrared SED is the placement of the polar dust clouds along the radial direction, i.e., the radial density profile.

\section{Appendix B \\ Sample and Data Compilation for Low-z Seyfert-1 nuclei}

\section{B.1. AGNs with HSR SED Observations}

We first constructed a sample of AGN with HSR measurements as follows. Asmus et al. (2014) presented a comprehensive mid-IR imaging atlas of 253 nearby AGNs with publicly available data from subarcsec-resolution observations carried out by ground-based $8 \mathrm{~m}$ class telescopes. We adopted this 

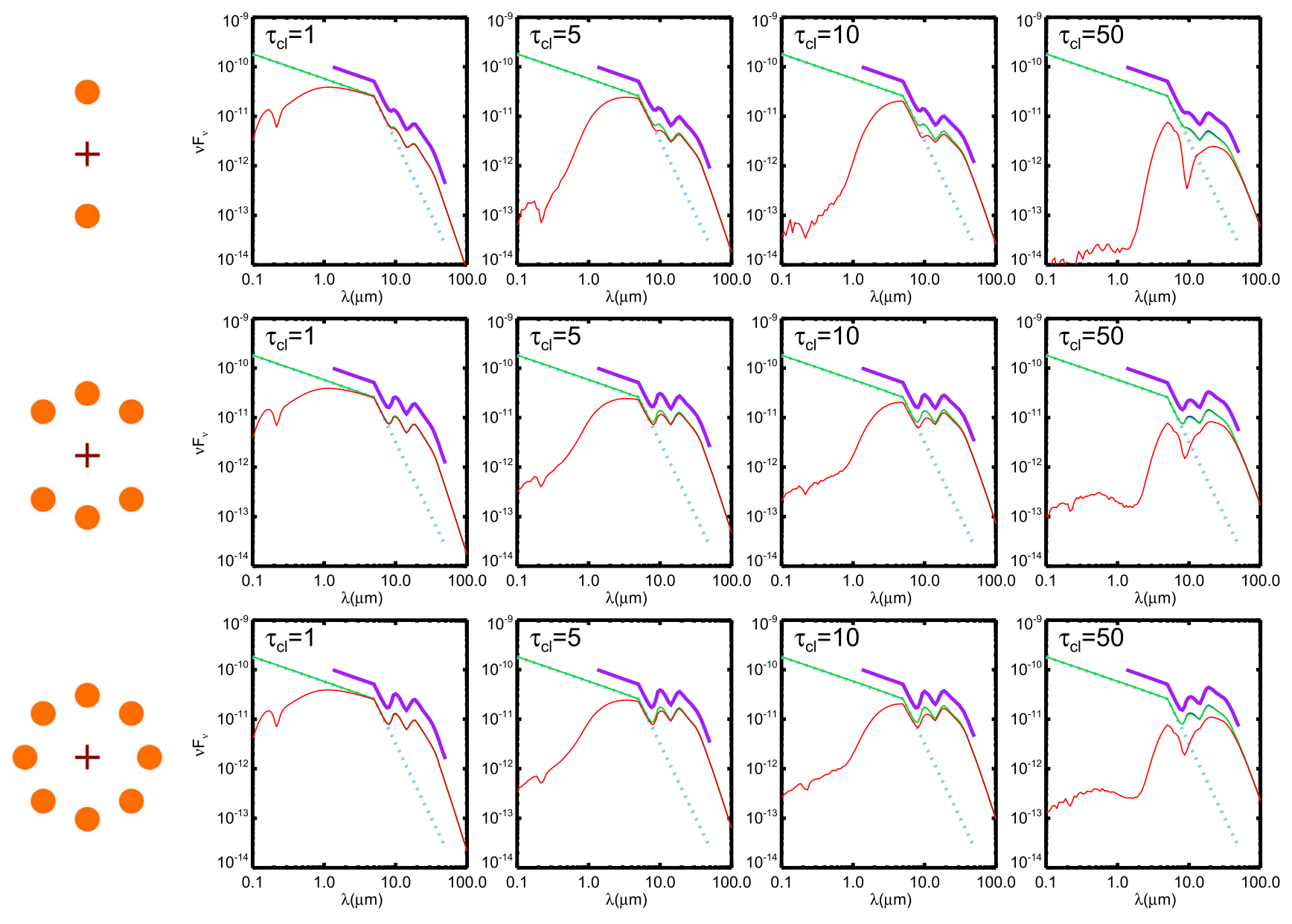

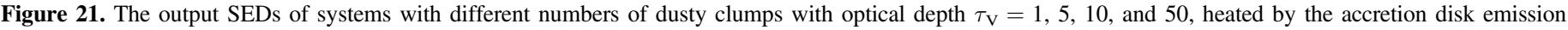

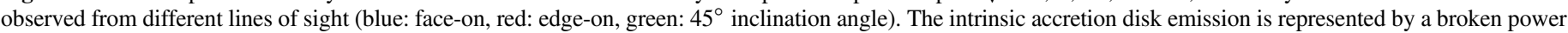

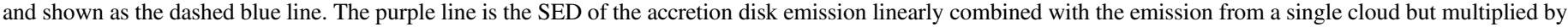
$N$ times, where $N$ is the number of the clouds in the corresponding system. We arbitrarily scaled the purple by two times for clarity.

atlas as the parent sample and selected all type-1, 1.2, 1.5, and 1n objects. To reduce the possibility of mid-IR host galaxy contamination, we cross-matched this sample with the ALLWISE source catalog (Wright et al. 2010) and compared the $12 \mu \mathrm{m}$ flux from ground-based subarcsec-resolution observation, $F_{g}(12 \mu \mathrm{m})$, and that from space-based $6 . " 5$ resolution WISE band 3 observation, $F_{s}(12 \mu \mathrm{m})$. The W3 flux is known to have a systematic bias that is color-dependent (Section 2.2 in Wright et al. 2010). Our objects have $f_{\nu} \propto \nu^{-\beta}$ with $\beta \sim 0-3$, which corresponds to a flux correction factor of $0.92-1.10$. Considering this and other uncertainties from, e.g., flux zeropoints and filter differences, we selected objects that satisfy $F(12 \mu \mathrm{m})_{g} / F(12 \mu \mathrm{m})_{s}>0.9$. In addition, we required that the source shape be consistent with a point source in all four WISE bands. Any objects falling within the extrapolated isophotal footprint of a 2MASS extended source have been removed to avoid strong stellar contamination in the near-IR. ${ }^{4}$ Since the SEDs of PG quasars whose SEDs have been studied in our previous work (Lyu et al. 2017), we removed them from this study.

Since we are also interested in the AGN polar dust emission, we included all the type-1 objects with mid-IR interferometry observations in López-Gonzaga et al. (2016) besides 3C 273

\footnotetext{
4 We removed any ALLWISE sources with ext_flg $=1,2,3$, and 5 .
}

(PG 1226+023). Finally, some well-known nearby Seyfert-1 nuclei with HSR IR SEDs in the literature (e.g., AlonsoHerrero et al. 2003; Ramos Almeida et al. 2009; Prieto et al. 2010; Fuller et al. 2016) are also included in this study. This sample is summarized in Table 3.

For the above sample selected from Asmus et al. (2014), we adopted the 2MASS profile-fit photometry and the WISE profile-fit photometry to sample the near-IR to mid-IR SED. The full width at half maximum (FWHM) of a typical 2MASS point-spread-function (PSF) is about 2.5 arcsec and the FWHM of the WISE band 1-3 is about 6 arcsec. We replace them with the higher spatial resolution data from, e.g., Spitzer/IRAC or $H S T /$ NICMOS if available. It is known that the ground-based mid-IR imaging could have unstable PSF (e.g., Radomski et al. 2008), thus we retained the WISE band $3(12 \mu \mathrm{m})$ flux. The FWHM of the WISE W4 band is about 12 arcsec, we replace that with Spitzer/MIPS 24 micron data wherever possible. Most of the other objects have (sub)arcsec resolution SEDs presented in the literature. We complemented the incomplete SEDs with either WISE or 2MASS photometry.

To further constrain the SED shape of the dust emission, we collected the mid-IR spectra for objects observed by Spitzer/ IRS. For the staring mode observations, we adopt the CASSIS products (Lebouteiller et al. 2011). For the mapping mode, we used CUBISM (Smith et al. 2007a) to reprocess the data, 

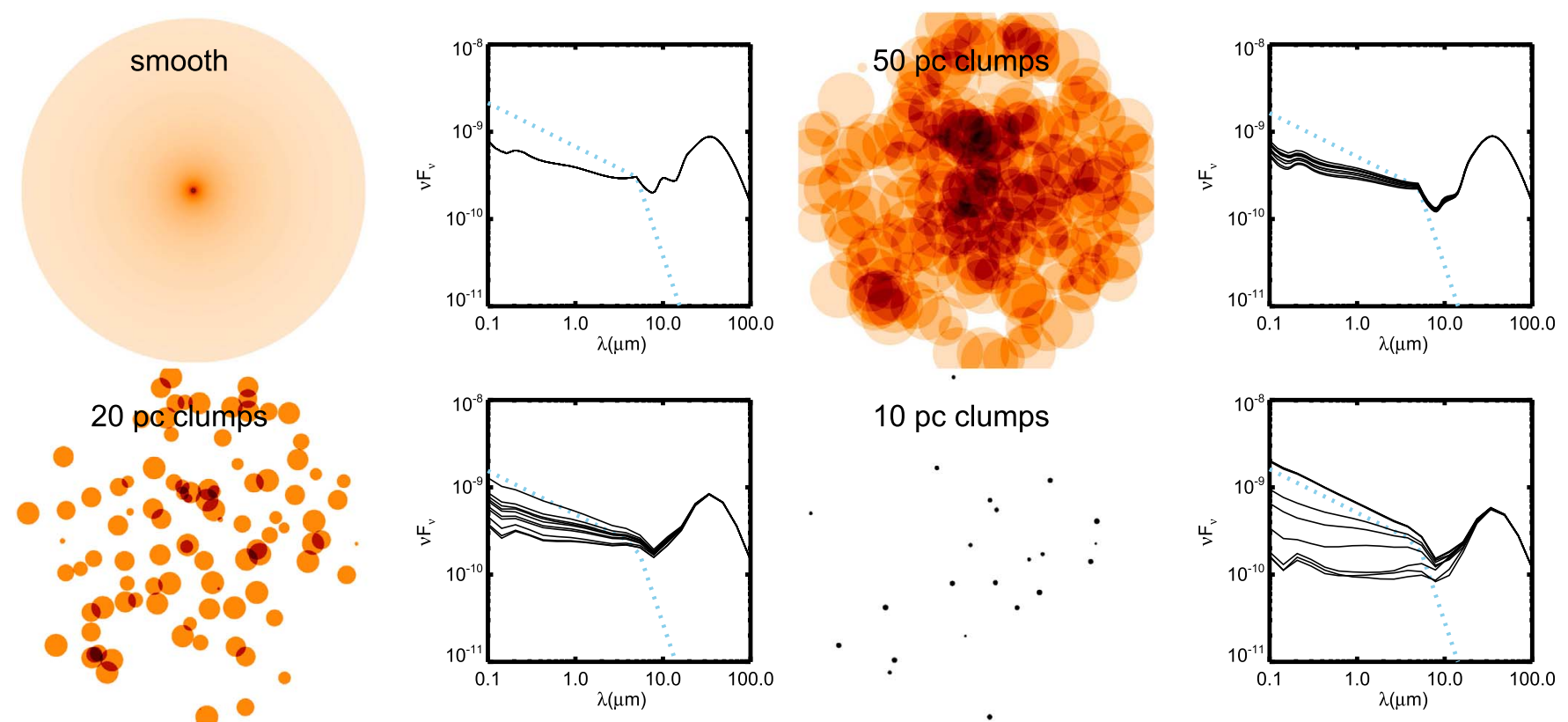

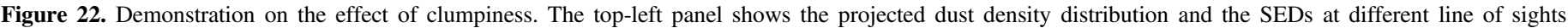

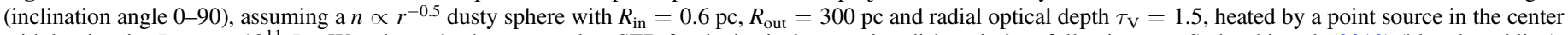

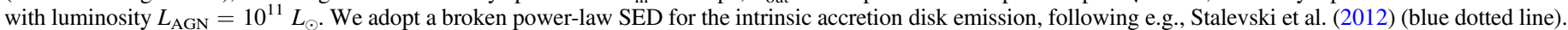

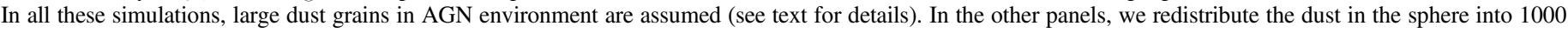

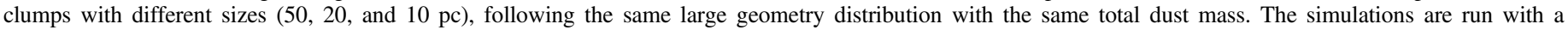
$100 \times 100 \times 100$ bin linear mesh of a box size $600 \times 600 \times 600 \mathrm{pc}$.

following the standard pipeline given in the software document.

\section{B.2. The SDSS-Spitzer/IRS Type-1 AGNS}

We adopted the spectral decomposition results of the Main Galaxy Sample (Strauss et al. 2002) in the SDSS Seventh Data Release (DR7; Abazajian et al. 2009) processed with the optical spectral data reduction pipeline developed by Hao et al. (2005). After subtracting the host galaxy continuum and the power-law AGN components, type-1 AGNs were selected as objects with the $\mathrm{H} \alpha$ broad component with FWHM $>1200$ $\mathrm{km} / \mathrm{s}$ from multi-Gaussian line-profile fit with a rest-frame $\mathrm{H} \alpha$ equivalent width $(\mathrm{EW})>3 \AA$. Due to the requirement of a direction of $\mathrm{H} \alpha$ information, the sample was limited to $z<0.33$ by the SDSS spectral coverage.

After compiling the SDSS Seyfert-1 sample, we crossmatched their SDSS coordinates with the Combined Atlas of Sources with Spitzer IRS Spectra (CASSIS, Lebouteiller et al. 2011) within a $3^{\prime \prime}$ search radius to get the mid-IR data. The CASSIS contains all Spitzer/IRS staring mode observations with enough signal for a useful spectrum. Since a strong host galaxy contamination in the mid-IR would make the interpretations ambiguous, only objects without evidence for strong aromatic features or silicate absorption(s) were selected. All of the sample have $11.3 \mu \mathrm{m}$ aromatic features with an EW less than $0.1 \mu \mathrm{m}$ from Spitzer/IRS spectral decomposition with a modified version of PAHFIT (Smith et al. 2007b), following Gallimore et al. (2010) to add an optically thin, warm dust component to reproduce the AGN silicate emission features (see their Section 4.1). Finally, we ended up with 33 SDSSSpitzer/IRS type-1 AGNs. We used the IDL routine DeblendIRS (Hernán-Caballero et al. 2015) to double check the level of star formation; the average luminosity contribution at $5.5-15 \mu \mathrm{m}$ is about $3 \%$ with the maximum value $<9 \%$.

To cover the full SED, we collected other multi-band photometry data for this sample. Seventeen objects $(\sim 52 \%)$ have X-ray observations from XMM-Newton or Chandra. We searched the GALEX, SDSS, 2MASS, and WISE archives for the corresponding UV/optical/IR photometric data. Over $80 \%$ of these objects are resolved in the 2MASS images; for them, we adopted the Standard Photometry with isophotal apertures based on the $K_{s} 20 \mathrm{mag} \operatorname{arcsec}^{-2}$ elliptical isophote from the 2MASS extended source catalog. For the WISE W1 $(\sim 3.4 \mu \mathrm{m})$ and W2 $(\sim 4.6 \mu \mathrm{m})$ bands, we used the scaled-2MASS-aperture photometry with the largest aperture as long as no other sources was included if the object was in the 2MASS extended source catalog and the WISE aperture photometry is larger than the default profile-fit photometry. We adopted the profile-fit photometry of the $\mathrm{J}, \mathrm{H}, \mathrm{K}, \mathrm{W} 1$, and $\mathrm{W} 2$ band in other cases.

\section{B.3. IR Light Curves from WISE/NEOWISE}

To address the infrared variabilities of these AGNs, we derived light curves based on the W1 and W2 band observations from the WISE (Wright et al. 2010) and the Near-Earth Object WISE Reactivation (NEOWISE-R; Mainzer et al. 2014) missions, following similar procedures in our previous work (Lyu et al. 2017). For most objects, the light curves cover seven to nine different epochs with 10-20 individual exposures for each epoch. We computed the mean photometry for each epoch and derived the maximum variability amplitudes. These results are included in Table 3.

\section{ORCID iDs}

Jianwei Lyu (1D https://orcid.org/0000-0002-6221-1829

George H. Rieke (i) https://orcid.org/0000-0003-2303-6519 


\section{References}

Abazajian, K. N., Adelman-McCarthy, J. K., Agüeros, M. A., et al. 2009, ApJS, 182, 543

Abrahamyan, H. V., Mickaelian, A. M., \& Knyazyan, A. V. 2015, A\&C, 10,99

Aitken, D. K., \& Roche, P. F. 1985, MNRAS, 213, 777

Alonso-Herrero, A., Quillen, A. C., Rieke, G. H., Ivanov, V. D., \& Efstathiou, A. 2003, AJ, 126, 81

Alonso-Herrero, A., Ramos Almeida, C., Mason, R., et al. 2011, ApJ, 736, 82 Antonucci, R. 1993, ARA\&A, 31, 473

Asmus, D., Hönig, S. F., \& Gandhi, P. 2016, ApJ, 822, 109

Asmus, D., Hönig, S. F., Gandhi, P., Smette, A., \& Duschl, W. J. 2014, MNRAS, 439, 1648

Assef, R. J., Eisenhardt, P. R. M., Stern, D., et al. 2015, ApJ, 804, 27

Assef, R. J., Kochanek, C. S., Brodwin, M., et al. 2010, ApJ, 713, 970

Assef, R. J., Stern, D., Kochanek, C. S., et al. 2013, ApJ, 772, 26

Auld, R., Bianchi, S., Smith, M. W. L., et al. 2013, MNRAS, 428, 1880

Baes, M., Davies, J. I., Dejonghe, H., et al. 2003, MNRAS, 343, 1081

Baes, M., Verstappen, J., De Looze, I., et al. 2011, ApJS, 196, 22

Ballantyne, D. R. 2008, ApJ, 685, 787

Baskin, A., \& Laor, A. 2018, MNRAS, 474, 1970

Behar, E. 2009, ApJ, 703, 1346

Bennett, C. L., Halpern, M., Hinshaw, G., et al. 2003, ApJS, 148, 1

Bianchi, S., Maiolino, R., \& Risaliti, G. 2012, AdAst, 2012, 782030

Bitsakis, T., Charmandaris, V., Appleton, P. N., et al. 2014, A\&A, 565, A25

Bock, J. J., Neugebauer, G., Matthews, K., et al. 2000, AJ, 120, 2904

Bongiorno, A., Merloni, A., Brusa, M., et al. 2012, MNRAS, 427, 3103

Bongiorno, A., Zamorani, G., Gavignaud, I., et al. 2007, A\&A, 472, 443

Braatz, J. A., Wilson, A. S., Gezari, D. Y., Varosi, F., \& Beichman, C. A. 1993, ApJL, 409, L5

Bressan, A., Granato, G. L., \& Silva, L. 1998, A\&A, 332, 135

Bressan, A., Panuzzo, P., Buson, L., et al. 2006, ApJL, 639, L55

Bruzual, G., \& Charlot, S. 2003, MNRAS, 344, 1000

Burtscher, L., Meisenheimer, K., Tristram, K. R. W., et al. 2013, A\&A, 558, A149

Cameron, M., Storey, J. W. V., Rotaciuc, V., et al. 1993, ApJ, 419, 136

Caputi, K. I. 2014, IJMPD, 23, 1430015

Cassarà, L. P., Piovan, L., Weiss, A., Salaris, M., \& Chiosi, C. 2013, MNRAS, 436, 2824

Collinson, J. S., Ward, M. J., Landt, H., et al. 2017, MNRAS, 465, 358

Comastri, A., Brunetti, G., Dallacasa, D., et al. 2003, MNRAS, 340, L52

Corral, A., Della Ceca, R., Caccianiga, A., et al. 2011, A\&A, 530, A42

Crenshaw, D. M., Kraemer, S. B., \& George, I. M. 2003, ARA\&A, 41, 117

Czerny, B., Li, J., Loska, Z., \& Szczerba, R. 2004, MNRAS, 348, L54

Díaz-Santos, T., Assef, R. J., Blain, A. W., et al. 2016, ApJL, 816, L6

Dicken, D., Tadhunter, C., Axon, D., et al. 2010, ApJ, 722, 1333

Dicken, D., Tadhunter, C., Morganti, R., et al. 2008, ApJ, 678, 712

Donley, J. L., Koekemoer, A. M., Brusa, M., et al. 2012, ApJ, 748, 142

Draine, B. T., \& Lee, H. M. 1984, ApJ, 285, 89

Efstathiou, A. 2006, MNRAS, 371, L70

Eisenhardt, P. R. M., Wu, J., Tsai, C.-W., et al. 2012, ApJ, 755, 173

Elvis, M., Hao, H., Civano, F., et al. 2012, ApJ, 759, 6

Elvis, M., Marengo, M., \& Karovska, M. 2002, ApJL, 567, L107

Elvis, M., Wilkes, B. J., McDowell, J. C., et al. 1994, ApJS, 95, 1

Fabian, A. C. 1999, MNRAS, 308, L39

Fan, L., Han, Y., Nikutta, R., Drouart, G., \& Knudsen, K. K. 2016, ApJ, 823, 107

Fan, L., Knudsen, K. K., Fogasy, J., \& Drouart, G. 2018, ApJL, 856, L5

Faucher-Giguère, C.-A., \& Quataert, E. 2012, MNRAS, 425, 605

Feltre, A., Hatziminaoglou, E., Fritz, J., \& Franceschini, A. 2012, MNRAS, 426, 120

Feruglio, C., Fiore, F., Carniani, S., et al. 2015, A\&A, 583, A99

Fitzpatrick, E. L. 1999, PASP, 111, 63

Fritz, J., Franceschini, A., \& Hatziminaoglou, E. 2006, MNRAS, 366, 767

Fuller, L., Lopez-Rodriguez, E., Packham, C., et al. 2016, MNRAS, 462, 2618

Gallimore, J. F., Yzaguirre, A., Jakoboski, J., et al. 2010, ApJS, 187, 172

García-González, J., Alonso-Herrero, A., Hernán-Caballero, A., et al. 2016, MNRAS, 458, 4512

Gaskell, C. M., \& Benker, A. J. 2007, arXiv:0711.1013

Gaskell, C. M., Goosmann, R. W., Antonucci, R. R. J., \& Whysong, D. H. 2004, ApJ, 616, 147

González-Lópezlira, R. A., Bruzual-A., G., Charlot, S., Ballesteros-Paredes, J., \& Loinard, L. 2010, MNRAS, 403, 1213

Gorjian, V., Werner, M. W., Jarrett, T. H., Cole, D. M., \& Ressler, M. E. 2004, ApJ, 605, 156
Goulding, A. D., Zakamska, N. L., Alexandroff, R. M., et al. 2018, ApJ, 856, 4 Groves, B., Dopita, M., \& Sutherland, R. 2006, A\&A, 458, 405

Güver, T., \& Özel, F. 2009, MNRAS, 400, 2050

Haas, M., Siebenmorgen, R., Pantin, E., et al. 2007, A\&A, 473, 369

Hall, P. B., Anderson, S. F., Strauss, M. A., et al. 2002, ApJS, 141, 267

Hamann, F., Zakamska, N. L., Ross, N., et al. 2017, MNRAS, 464, 3431

Hanish, D. J., Capak, P., Teplitz, H. I., et al. 2015, ApJS, 217, 17

Hao, H., Elvis, M., Civano, F., et al. 2010, ApJL, 724, L59

Hao, H., Elvis, M., Civano, F., \& Lawrence, A. 2011, ApJ, 733, 108

Hao, L., Strauss, M. A., Tremonti, C. A., et al. 2005, AJ, 129, 1783

Hao, L., Weedman, D. W., Spoon, H. W. W., et al. 2007, ApJL, 655, L77

Heckman, T. M., \& Best, P. N. 2014, ARA\&A, 52, 589

Hernán-Caballero, A., Alonso-Herrero, A., Hatziminaoglou, E., et al. 2015, ApJ, 803, 109

Hernán-Caballero, A., \& Hatziminaoglou, E. 2011, MNRAS, 414, 500

Hoenig, S. F. 2013, arXiv:1301.1349

Höfner, S. 2008, A\&A, 491, L1

Hönig, S. F., \& Kishimoto, M. 2010, A\&A, 523, A27

Hönig, S. F., \& Kishimoto, M. 2017, ApJL, 838, L20

Hönig, S. F., Kishimoto, M., Antonucci, R., et al. 2012, ApJ, 755, 149

Hönig, S. F., Kishimoto, M., Tristram, K. R. W., et al. 2013, ApJ, 771, 87

Hopkins, P. F., Hayward, C. C., Narayanan, D., \& Hernquist, L. 2012, MNRAS, 420, 320

Hopkins, P. F., Richards, G. T., \& Hernquist, L. 2007, ApJ, 654, 731

Hopkins, P. F., Strauss, M. A., Hall, P. B., et al. 2004, AJ, 128, 1112

Hopkins, P. F., Torrey, P., Faucher-Giguère, C.-A., Quataert, E., \& Murray, N. 2016, MNRAS, 458, 816

Hopkins, P. F., Younger, J. D., Hayward, C. C., Narayanan, D., \& Hernquist, L. 2010, MNRAS, 402, 1693

Ivezic, Z., \& Elitzur, M. 1997, MNRAS, 287, 799

Ivezic, Z., Nenkova, M., Heymann, F., \& Elitzur, M. 2017, User Manual for DUSTY (V4)

Jiang, L., Fan, X., Brandt, W. N., et al. 2010, Natur, 464, 380

Johnson, S. P., Wilson, G. W., Tang, Y., \& Scott, K. S. 2013, MNRAS, 436, 2535

Jones, S. F., Blain, A. W., Lonsdale, C., et al. 2015, MNRAS, 448, 3325

Jones, S. F., Blain, A. W., Stern, D., et al. 2014, MNRAS, 443, 146

Kawaguchi, T., \& Mori, M. 2010, ApJL, 724, L183

Kim, D., Im, M., Kim, J. H., et al. 2015, ApJS, 216, 17

King, A. 2003, ApJL, 596, L27

Kirkpatrick, A., Pope, A., Sajina, A., et al. 2015, ApJ, 814, 9

Kishimoto, M., Hönig, S. F., Antonucci, R., et al. 2009, A\&A, 507, L57

Kishimoto, M., Hönig, S. F., Beckert, T., \& Weigelt, G. 2007, A\&A, 476, 713

Krawczyk, C. M., Richards, G. T., Mehta, S. S., et al. 2013, ApJS, 206, 4

Lacy, M., Petric, A. O., Sajina, A., et al. 2007, AJ, 133, 186

Lacy, M., Storrie-Lombardi, L. J., Sajina, A., et al. 2004, ApJS, 154, 166

Landt, H., Buchanan, C. L., \& Barmby, P. 2010, MNRAS, 408, 1982

Lani, C., Netzer, H., \& Lutz, D. 2017, MNRAS, 471, 59

Laor, A., \& Draine, B. T. 1993, ApJ, 402, 441

Lawrence, A. 1991, MNRAS, 252, 586

Lebouteiller, V., Barry, D. J., Spoon, H. W. W., et al. 2011, ApJS, 196, 8

Leftley, J. H., Tristram, K. R. W., Hönig, S. F., et al. 2018, ApJ, 862, 17

Leipski, C., Meisenheimer, K., Walter, F., et al. 2014, ApJ, 785, 154

López-Gonzaga, N., Burtscher, L., Tristram, K. R. W., Meisenheimer, K., \& Schartmann, M. 2016, A\&A, 591, A47

Lusso, E., Hennawi, J. F., Comastri, A., et al. 2013, ApJ, 777, 86

Lyu, J., Hao, L., \& Li, A. 2014, ApJL, 792, L9

Lyu, J., \& Rieke, G. H. 2017, ApJ, 841, 76

Lyu, J., Rieke, G. H., \& Alberts, S. 2016, ApJ, 816, 85

Lyu, J., Rieke, G. H., \& Shi, Y. 2017, ApJ, 835, 257

Ma, Z., \& Yan, H. 2015, ApJ, 811, 58

Mainzer, A., Bauer, J., Cutri, R. M., et al. 2014, ApJ, 792, 30

Maiolino, R., Marconi, A., \& Oliva, E. 2001a, A\&A, 365, 37

Maiolino, R., Marconi, A., Salvati, M., et al. 2001b, A\&A, 365, 28

Maiolino, R., Shemmer, O., Imanishi, M., et al. 2007, A\&A, 468, 979

Mathis, J. S., Rumpl, W., \& Nordsieck, K. H. 1977, ApJ, 217, 425

Matsuta, K., Gandhi, P., Dotani, T., et al. 2012, ApJ, 753, 104

Meléndez, M., Mushotzky, R. F., Shimizu, T. T., Barger, A. J., \& Cowie, L. L. 2014, ApJ, 794, 152

Mor, R., Netzer, H., \& Elitzur, M. 2009, ApJ, 705, 298

Mor, R., \& Trakhtenbrot, B. 2011, ApJL, 737, L36

Moshir, M., Kopan, G., Conrow, T., et al. 1990, BAAS, 22, 1325

Murray, N., Quataert, E., \& Thompson, T. A. 2005, ApJ, 618, 569

Nenkova, M., Sirocky, M. M., Ivezić, Ž, \& Elitzur, M. 2008a, ApJ, 685, 147

Nenkova, M., Sirocky, M. M., Nikutta, R., Ivezić, Ž, \& Elitzur, M. 2008b, ApJ, 685,160 
Netzer, H. 2015, ARA\&A, 53, 365

Netzer, H., Shemmer, O., Maiolino, R., et al. 2004, ApJ, 614, 558

Nikutta, R., Elitzur, M., \& Lacy, M. 2009, ApJ, 707, 1550

Oliver, S. J., Bock, J., Altieri, B., et al. 2012, MNRAS, 424, 1614

Pe'er, A. 2014, SSRv, 183, 371

Peng, Z., Gu, Q., Melnick, J., \& Zhao, Y. 2006, A\&A, 453, 863

Piconcelli, E., Jimenez-Bailón, E., Guainazzi, M., et al. 2005, A\&A, 432, 15

Piovan, L., Tantalo, R., \& Chiosi, C. 2003, A\&A, 408, 559

Polletta, M., Tajer, M., Maraschi, L., et al. 2007, ApJ, 663, 81

Pollo, A., Rybka, P., \& Takeuchi, T. T. 2010, A\&A, 514, A3

Prieto, M. A., Reunanen, J., Tristram, K. R. W., et al. 2010, MNRAS, 402, 724

Raban, D., Jaffe, W., Röttgering, H., Meisenheimer, K., \& Tristram, K. R. W. 2009, MNRAS, 394, 1325

Radomski, J. T., Packham, C., Levenson, N. A., et al. 2008, ApJ, 681, 141

Ramos Almeida, C., Levenson, N. A., Rodríguez Espinosa, J. M., et al. 2009, ApJ, 702, 1127

Rampazzo, R., Panuzzo, P., Vega, O., et al. 2013, MNRAS, 432, 374

Reunanen, J., Prieto, M. A., \& Siebenmorgen, R. 2010, MNRAS, 402, 879

Revalski, M., Crenshaw, D. M., Kraemer, S. B., et al. 2018, ApJ, 856, 46

Ricci, C., Assef, R. J., Stern, D., et al. 2017a, ApJ, 835, 105

Ricci, C., Trakhtenbrot, B., Koss, M. J., et al. 2017b, Natur, 549, 488

Richards, G. T., Hall, P. B., Vanden Berk, D. E., et al. 2003, AJ, 126, 1131

Richards, G. T., Lacy, M., Storrie-Lombardi, L. J., et al. 2006, ApJS, 166, 470

Rieke, G. H., Alonso-Herrero, A., Weiner, B. J., et al. 2009, ApJ, 692, 556

Roseboom, I. G., Lawrence, A., Elvis, M., et al. 2013, MNRAS, 429, 1494

Ross, N. P., Hamann, F., Zakamska, N. L., et al. 2015, MNRAS, 453, 3932

Roth, N., Kasen, D., Hopkins, P. F., \& Quataert, E. 2012, ApJ, 759, 36

Rudy, R. J., Levan, P. D., \& Rodriguez-Espinosa, J. M. 1982, AJ, 87, 598

Rujopakarn, W., Rieke, G. H., Weiner, B. J., et al. 2013, ApJ, 767, 73

Sanders, D. B., Phinney, E. S., Neugebauer, G., Soifer, B. T., \& Matthews, K. 1989, ApJ, 347, 29

Sanders, D. B., Soifer, B. T., Elias, J. H., et al. 1988a, ApJ, 325, 74

Sanders, D. B., Soifer, B. T., Elias, J. H., Neugebauer, G., \& Matthews, K. 1988b, ApJL, 328, L35

Sargsyan, L., Weedman, D., Lebouteiller, V., et al. 2011, ApJ, 730, 19

Schlafly, E. F., \& Finkbeiner, D. P. 2011, ApJ, 737, 103

Schlegel, D. J., Finkbeiner, D. P., \& Davis, M. 1998, ApJ, 500, 525

Schweitzer, M., Groves, B., Netzer, H., et al. 2008, ApJ, 679, 101

Scott, A. E., \& Stewart, G. C. 2014, MNRAS, 438, 2253

Scoville, N. Z., Evans, A. S., Thompson, R., et al. 2000, AJ, 119, 991

Shang, Z., Brotherton, M. S., Wills, B. J., et al. 2011, ApJS, 196, 2

Shao, Z., Jiang, B. W., \& Li, A. 2017, ApJ, 840, 27

Shi, Y., Rieke, G. H., Ogle, P. M., Su, K. Y. L., \& Balog, Z. 2014, ApJS, 214,23
Shi, Y., Rieke, G. H., Smith, P., et al. 2010, ApJ, 714, 115

Shimizu, T. T., Davies, R. I., Koss, M., et al. 2018, ApJ, 856, 154

Shimizu, T. T., Meléndez, M., Mushotzky, R. F., et al. 2016, MNRAS, 456, 3335

Siebenmorgen, R., Heymann, F., \& Efstathiou, A. 2015, A\&A, 583, A120

Silva, L., Granato, G. L., Bressan, A., \& Danese, L. 1998, ApJ, 509, 103

Simpson, C. 2005, MNRAS, 360, 565

Skrutskie, M. F., Cutri, R. M., Stiening, R., et al. 2006, AJ, 131, 1163

Smith, J. D. T., Armus, L., Dale, D. A., et al. 2007a, PASP, 119, 1133

Smith, J. D. T., Draine, B. T., Dale, D. A., et al. 2007b, ApJ, 656, 770

Spinoglio, L., Malkan, M. A., Rush, B., Carrasco, L., \& Recillas-Cruz, E. 1995, ApJ, 453, 616

Stalevski, M., Asmus, D., \& Tristram, K. R. W. 2017, MNRAS, 472, 3854

Stalevski, M., Fritz, J., Baes, M., Nakos, T., \& Popović, L. Č. 2012, MNRAS, 420, 2756

Stalevski, M., Ricci, C., Ueda, Y., et al. 2016, MNRAS, 458, 2288

Stern, D., Assef, R. J., Benford, D. J., et al. 2012, ApJ, 753, 30

Stern, D., Eisenhardt, P., Gorjian, V., et al. 2005, ApJ, 631, 163

Stern, D., Lansbury, G. B., Assef, R. J., et al. 2014, ApJ, 794, 102

Strauss, M. A., Weinberg, D. H., Lupton, R. H., et al. 2002, AJ, 124, 1810

Sturm, E., Schweitzer, M., Lutz, D., et al. 2005, ApJL, 629, L21

Teplitz, H. I., Capak, P., Brooke, T., et al. 2010, in ASP Conf. Ser. 434, Astronomical Data Analysis Software and Systems XIX, ed. Y. Mizumoto, K.-I. Morita, \& M. Ohishi (San Francisco, CA: ASP), 437

Thompson, T. A., Quataert, E., \& Murray, N. 2005, ApJ, 630, 167

Treister, E., Krolik, J. H., \& Dullemond, C. 2008, ApJ, 679, 140

Tristram, K. R. W., Burtscher, L., Jaffe, W., et al. 2014, A\&A, 563, A82

Urry, C. M., \& Padovani, P. 1995, PASP, 107, 803

Villaume, A., Conroy, C., \& Johnson, B. D. 2015, ApJ, 806, 82

Vito, F., Brandt, W. N., Stern, D., et al. 2018, MNRAS, 474, 4528

Wada, K. 2012, ApJ, 758, 66

Wada, K., Papadopoulos, P. P., \& Spaans, M. 2009, ApJ, 702, 63

Weingartner, J. C., \& Murray, N. 2002, ApJ, 580, 88

Wright, E. L., Eisenhardt, P. R. M., Mainzer, A. K., et al. 2010, AJ, 140, 1868

Wu, J., Jun, H. D., Assef, R. J., et al. 2018, ApJ, 852, 96

Wu, J., Tsai, C.-W., Sayers, J., et al. 2012, ApJ, 756, 96

Xie, Y., Li, A., \& Hao, L. 2017, ApJS, 228, 6

Xu, L., Rieke, G. H., Egami, E., et al. 2015, ApJS, 219, 18

Yamamura, I., Makiuti, S., Ikeda, N., et al. 2010, yCat, 2298, 0

York, D. G., Adelman, J., Anderson, J. E., Jr., et al. 2000, AJ, 120, 1579

Zakamska, N. L., Hamann, F., Pâris, I., et al. 2016, MNRAS, 459, 3144

Zakamska, N. L., Schmidt, G. D., Smith, P. S., et al. 2005, AJ, 129, 1212

Zhang, K., Wang, T.-G., Yan, L., \& Dong, X.-B. 2013, ApJ, 768, 22 